\title{
How Important are Inflation Expectations for the Nominal Yield Curve?*
}

\author{
Roberto Gómez-Cram \\ University of Pennsylvania
}

\author{
Amir Yaron \\ University of Pennsylvania \\ NBER
}

This version: November 2017

\begin{abstract}
Less than you think. Macro-finance term structure models rely too heavily on the volatility of expected inflation news as a source for variations in nominal yield shocks. This paper develops and estimates a model featuring inflation non-neutrality and preference shocks. Stochastic volatility of inflation and consumption govern bond risk premia movements, while preference shocks generate volatile nominal yields. The model accounts for key bond market features, without resorting to an overly dominating expected inflation channel. The estimation shows that preference shocks are correlated with market distress factors, and that in the last two decades, inflation-related risks played a secondary role. (JEL C11, C32, C58, E43, E44, G12)
\end{abstract}

${ }^{*}$ The Wharton School, University of Pennsylvania, 3620 Locust Walk, Philadelphia, PA 19104-6367. E-mail: rogo@wharton.upenn.edu (Roberto Gómez-Cram), and yarona@wharton.upenn.edu (Amir Yaron).We thank Mete Kilic, Frank Schorfheide, Dongho Song, and seminar participants at Central Bank of Mexico, FMA, Northwestern University and Wharton. The usual disclaimer applies. Yaron thanks financial support from the Rodney White and Jacobs Levy centers. 


\section{Introduction}

Macro-finance term structure models are designed to interpret and quantify the economic mechanisms underlying the substantial variation in nominal yields and risk premia in bond markets. To this end, economists have used long-run risk (e.g., Piazzesi and Schneider 2007; Bansal and Shaliastovich 2013; Song 2017), New Keynesian (e.g., Rudebusch and Swanson 2012; Kung 2015), and habit formation (e.g., Wachter 2006) models. However, in order to match the empirical volatility of nominal yields these models rely on expected inflation news that are too volatile relative to the data (see Duffee (2016) for extended discussion). In the data, the variance of expected inflation news accounts for about 20 percent of the variance of nominal yield shocks, which is strongly at odds with model-implied ratios which often exceed 100 percent 1

In this paper, we develop and estimate a nonlinear Bayesian state-space macro-finance endowment model that accounts for key bond market features, without resorting to an expected inflation channel that overly dominates the variation in nominal yield shocks. The model features recursive preferences, inflation non-neutrality, multiple stochastic volatility processes, time preference shocks, and time-aggregation of consumption. Specifically, we build on the long-run risks setup of Bansal and Shaliastovich (2013) and Schorfheide, Song, and Yaron (2017) in which time variation in expected consumption and inflation and their respective volatilities are key in accounting for bond risk premia dynamics and matching standard bond market moments. The shocks to time rate of preference, on the other hand, primarily affect real rate fluctuations and thus limit the role of expected inflation shocks in driving nominal yield innovations. To assess the empirical validity of these different channels, we estimate the model using a Bayesian MCMC particle filter approach. To the best of our knowledge, this model is the first one that does not rely too heavily on the volatility of inflation expectations to fit the nominal yield curve.

The model can account for myriad of macro and bond market features. Specifically, the model is able to match standard moments such as the unconditional level, slope, and standard deviation of nominal yields. In addition, it generates sizable variation in bond risk premia, that is, it is able

\footnotetext{
${ }^{1}$ For example, in New Keynesian and Long Run Risks Models these variance ratios can exceed 100 percent. While habit formation models fare somewhat better with variance ratios of around 50 percent, they lack a consistent channel for predictability in bond and currency returns (e.g., Wachter (2006) requires a counter-cyclical interest rates to account for bond return predictability, while Verdelhan (2010) relies on a pro-cyclical interest rates to account for the violations of uncovered interest parity in currency markets).
} 
to generate time-varying term premium that mimics the estimates based on reduced-form Gaussian affine term structure models (e.g., Adrian, Crump, and Moench 2013; Kim and Wright 2005) without distorting its macroeconomic fit or requiring a large coefficient of relative risk aversion (roughly 8). Moreover, it quantitatively matches the evidence of bond return predictability documented in Cochrane and Piazzesi (2005) and the failure of the expectation hypothesis, as identified in Campbell and Shiller (1991). Importantly, the model is able to match these term structure features with expected inflation innovations that, as in the data, do not dominate variations in yield news. In this regard, we show that the inflation variance ratios (for different horizons), defined as the variance of expected inflation news relative to the variance of yield shocks, are indeed smaller than existing structural models ascribe, yet the point estimates are larger than initially viewed once appropriate econometrics accounts for stochastic volatility in inflation and bond yields $2^{2}$

The model estimation allows us to decompose the risk premia into their economic components in real time. These estimates suggest that over time inflation-related risks, while important, have had a declining effect on risk premia movements in bond yields. Specifically, while the term premium and expected excess bond returns are on average positive, primarily due to inflation-related risk premia, they have been negative for the last decade due to variations in real uncertainties.

The model features two key mechanisms. The first and most important one, builds on the longrun risks model of Bansal and Yaron (2004) and follows the formulation in Bansal and Shaliastovich (2013) in assuming that consumption growth and inflation contain a small predictable component with time-varying conditional volatilities. With preferences for early resolution of uncertainty, variations in bond risk premia are driven entirely by these volatilities. Specifically, an increase in real volatility (uncertainty) lowers bond risk premia, whereas nominal volatility raises it, provided that expected inflation shocks are negatively correlated with expected consumption growth (inflation nonneutrality). In such a setting, nominal shocks are priced, which makes long-term bonds particularly risky since innovations that produce a persistent increase in expected inflation generate persistently low real yields and low expected consumption growth. This mechanism is important for generating

\footnotetext{
${ }^{2}$ To compute the data-implied inflation variance ratios for long horizons, we rely on a statistical model of inflation and bond yields. We extend the unconditional inflation variance ratios proposed by Duffee (2016) by modeling the dynamics of conditional inflation variance ratios. Accounting for conditional information (stochastic volatility) improves the statistical model's fit and better aligns the data and structural model implied inflation variance moments. The fraction of yield news variation that is attributable to variation in inflation news is higher when conditional information is accounted for. Nevertheless, these larger estimates still pose a challenge for existing macro-finance models that rely heavily on the expected inflation channel for yield news innovations.
} 
a positive slope in the nominal yield curve and crucial for movements and predictability of bond risk premia.

The time preference shocks provide the second mechanism. In this model, time preference shocks arise from stochastic changes in agents' discount factor and determine how they trade off current versus future utility. Albuquerque, Eichenbaum, Luo, and Rebelo (2016), and Schorfheide et al. (2017), also include time preference shocks in their model specification to better account for the risk free rate dynamics and its relatively low correlation with consumption growth $-\mathrm{a}$ feature preserved in this model. Furthermore, with preference for early resolution of uncertainty, the exposure to these shocks is an increasing function of the bond's maturity and generate an upwardsloping real yield curve. Hence, it complements the first mechanism in producing an upward-sloping nominal yield curve. Time preference shocks also generate volatile and persistent fluctuations in expected short-term real rates. This in turn allows the model to match the empirical volatility of nominal yield shocks without resorting to innovations in inflation expectations that vary too much relative to the data. However, it is important to recognize that although time preference shocks help generate news about real rates, they deteriorate the model implications for bond return predictability. This crucial trade-off is one important dimension the estimation of the model is addressing.

Time preference shocks determine the attitudes of the representative household towards savings, and are by assumption, one that we subsequently show is supported empirically, orthogonal to the real economy. Interestingly, and in line with this reasoning, the filtered time preference shocks series is highly correlated with the adjusted National Financial Conditions Index published by the Federal Reserve of Chicago, which measures risk, liquidity, and leverage in money, debt, and equity markets that is uncorrelated with economic conditions 3

The model contains several other ingredients such as time-varying volatilities in the iid component of consumption growth and inflation, and time-aggregation of consumption. These features are negligible for the nominal yield curve but are important for the tracking the macroeconomic series. Moreover, monthly measurement errors in the process of consumption that average out at the quarterly frequency aid the identification of the persistent parameter of expected consumption

\footnotetext{
${ }^{3}$ The Adjusted National Financial Conditions Index is provided by the Federal Reserve Bank of Chicago published at https: //alfred.stlouisfed.org/series?seid=ANFCI.
} 
growth. Importantly, we show that these features lead to remarkably similar estimates of macroeconomic series when bond yields are or are not used in the estimation 4 Finally, we show that there is enough information in the macroeconomic series, over the sample period 1960 to 2014, to identify a significant negative non-neutral effect of inflation on growth.

This paper is organized as follows. In Section 2, we introduce the model and cast it into a state-space form. Section 3 describes the Metropolis-Hastings sampler used for Bayesian inference, the data set, and prior and posterior distribution of model parameters. Section 4 analyzes the importance of inflation expectations for the nominal yield curve. Finally, Section 5 provides concluding remarks.

\section{Model specification}

In this section, we present the macro-finance model and cast it into a state-space form. In Section 2.1. we describe the preference of the representative household. In Section 2.2, we present the exogenous dynamics of consumption growth and inflation. In Section 2.3, we characterize the model solution and the equilibrium nominal yield curve. Finally, in Section 2.4, we cast the model into a state-space form.

\section{$2.1 \quad$ Preferences}

The representative household has Epstein and Zin (1989) recursive preferences and maximizes lifetime utility which is a function of current utility and the certainty equivalent of future utility $V_{t+1}^{*}$ :

$$
V_{t}=\max _{C_{t}}\left[(1-\delta) \lambda_{t} C_{t}^{1-\frac{1}{\psi}}+\delta\left(V_{t+1}^{*}\right)^{1-\frac{1}{\psi}}\right]^{\frac{1}{1-\frac{1}{\psi}}}
$$

where $C_{t}$ denotes consumption at time $t, \psi$ is intertemporal elasticity of substitution, $\delta$ is the discount factor, and $\lambda_{t}$ denotes a time-varying preference parameter. The certainty equivalent of future utility is the guaranteed value of lifetime utility at time $t+1$ given by $V_{t+1}^{*}=\left(E_{t} V_{t+1}^{1-\gamma}\right)^{\frac{1}{1-\gamma}}$, where the parameter $\gamma$ represents the coefficient of relative risk aversion.

\footnotetext{
${ }^{4}$ This result is important as it shows that the parameter estimates are not driven to merely fit the bond yields or are fundamentally disconnected from macroeconomic aggregates. Adding the bond prices primarily reduces the credible intervals of the parameter estimates.
} 
As in Albuquerque et al. (2016) and Schorfheide et al. (2017), we also allow for time preference shocks to the time rate of preferences given by $x_{\lambda, t}=\log \left(\lambda_{t+1}\right)-\log \left(\lambda_{t}\right)$, which determines how the household trades off current versus future utility. These shocks evolve according to:

$$
x_{\lambda, t+1}=\rho_{\lambda} x_{\lambda, t}+\sigma_{\lambda} \eta_{\lambda, t+1} \quad \text { with } \quad \eta_{\lambda, t+1} \sim i . i . d . N(0,1)
$$

and can be thought of as demand shocks that capture changes in the household attitudes towards savings. The representative household is subject to the following budget constraint: $W_{t+1}=\left(W_{t}-\right.$ $\left.C_{t}\right) R_{c, t+1}$, where $W_{t}$ denotes its wealth at time $t$ and $R_{c, t+1}$ is the return on an asset that pays aggregate consumption as dividends.

\subsection{Consumption and inflation dynamics}

The exogenous dynamics of the logarithm of consumption growth, $\Delta c_{t+1}=\log \left(C_{t+1}\right)-\log \left(C_{t}\right)$, and the logarithm of the inflation rate, $\pi_{t+1}$, are written as follows:

$$
\begin{aligned}
\Delta c_{t+1} & =\mu_{c}+x_{c, t}+\sigma_{c, t} \eta_{c, t+1} \\
\pi_{t+1} & =\mu_{\pi}+x_{\pi, t}+\sigma_{\pi, t} \eta_{\pi, t+1}
\end{aligned}
$$

where the process of conditional expectations is given by

$$
\begin{aligned}
& x_{c, t+1}=\rho_{c c} x_{c, t}+\rho_{c \pi} x_{\pi, t}+\sigma_{x c, t} \eta_{x c, t+1} \\
& x_{\pi, t+1}=\quad+\rho_{\pi \pi} x_{\pi, t}+\sigma_{x \pi, t} \eta_{x \pi, t+1}
\end{aligned}
$$

and volatilities evolve according to exponential Gaussian processes

$$
\sigma_{i, t}=\varphi_{i} \sigma \exp \left(h_{i, t}\right), \quad \text { with } \quad h_{i, t+1}=\rho_{h_{i}} h_{i, t}+\sigma_{h_{i}} \omega_{i, t+1}
$$

We normalize $\varphi_{c}=1$ and all innovations are distributed according to

$$
\eta_{i, t+1}, \quad \omega_{i, t+1}, \quad \epsilon_{i, t+1} \sim i . i . d . N(0,1) \quad \text { for } \quad i=\{c, \pi, x c, x \pi\}
$$

Specification (3) is based on Bansal and Yaron (2004), Piazzesi and Schneider (2007), and 
Bansal and Shaliastovich (2013), and decomposes real consumption growth and inflation into predictable $\left(x_{c, t}, x_{\pi, t}\right)$ and transitory $\left(\sigma_{c, t} \eta_{c, t+1}, \sigma_{\pi, t} \eta_{\pi, t+1}\right)$ components. We assume that the predictable components, that is expected consumption growth, $x_{c}$, and expected inflation, $x_{\pi}$, follow a bivariate $\operatorname{VAR}(1)$ process, with time-varying volatilities given by $\sigma_{x c, t}^{2}$ and $\sigma_{x \pi, t}^{2}$, respectively. We allow for expected inflation to directly feed into expected consumption growth via the parameter $\rho_{c \pi}$. This parameter characterizes the nonneutrality of inflation. In contrast to Bansal and Shaliastovich (2013), we consider four separate volatility processes. The long-run stochastic volatilities $\sigma_{x c, t}^{2}$ and $\sigma_{x \pi, t}^{2}$ are the drivers of bond risk premia, while the short-run volatilities $\sigma_{c, t}^{2}$ and $\sigma_{\pi, t}^{2}$ are negligible for bond prices, but are important for tracking the consumption growth and inflation series. Furthermore, the logarithm of the volatility process is assumed to be normal, which guarantees that $\sigma_{i, t}$ is positive throughout.

\subsection{Model solution}

The Euler equation associated with any continuous real return, $r_{t+1}$, can be written as

$$
E_{t}\left[\exp \left(m_{t+1}+r_{t+1}\right)\right]=1
$$

where the logarithm of the real stochastic discount factor (SDF) implied by the assumed household preferences is

$$
m_{t+1}=\theta \log \delta+\theta x_{\lambda, t}-\frac{\theta}{\psi} \Delta c_{t+1}+(\theta-1) r_{c, t+1} \quad \text { with } \quad \theta=\frac{1-\gamma}{1-\frac{1}{\psi}}
$$

Note that when $\gamma=\frac{1}{\psi}$ the utility function is of the constant relative risk aversion form and the SDF becomes independent of the log return on the consumption claim denoted by $r_{c, t+1}=\log \left(R_{c, t+1}\right)$. To price nominal payoffs it is useful to specify the nominal discount factor which is equal to the real one minus the inflation rate:

$$
m_{t+1}^{\$}=m_{t+1}-\pi_{t+1}
$$

To entertain an analytical model solution we use two different approximations. The first, proposed by Campbell and Shiller (1988), involves a log-linear Taylor expansion $r_{c, t+1} \approx \kappa_{0}+$ 
$\kappa_{1} p c_{t+1}-p c_{t}+\Delta c_{t+1}$, where $\kappa_{0}$ and $\kappa_{1}$ are constants determined endogenously by the unconditional mean of the price-consumption ratio, $p c_{t}$, given in Appendix A.1. To obtained an analytical solution for asset prices, the second approximation follows Schorfheide et al. (2017) and takes a linear approximation of the volatility process around the unconditional mean of $h_{i}$, given by $\sigma_{i, t+1}^{2} \approx \sigma_{i, 0}^{2}+$ $\nu_{i}\left(\sigma_{i, t}^{2}-\sigma_{i, 0}^{2}\right)+\sigma_{\omega_{i}} \omega_{i, t+1}$ for $i=\{c, \pi, x c, x \pi\}$ with $\nu_{i}=\rho_{h_{i}}, \sigma_{i, 0}^{2}=\left(\varphi_{i} \sigma\right)^{2}$, and $\sigma_{\omega_{i}}=2\left(\varphi_{i} \sigma\right)^{2} \sigma_{h_{i}}{ }^{5}$

The first approximation allows us to write innovations to the real SDF as a linear combination in shocks,

$$
\begin{aligned}
m_{t+1}-E_{t} m_{t+1} & =\underbrace{-\lambda_{c} \sigma_{c, t} \eta_{c, t+1}}_{\text {short-run consumption risk }} \underbrace{-\lambda_{\pi} \sigma_{\pi, t} \eta_{\pi, t+1}}_{\text {short-run inflation risk }} \underbrace{-\lambda_{\lambda} \sigma_{\lambda, t} \eta_{\lambda, t+1}}_{\text {preference risk }} \\
& \underbrace{-\lambda_{x c} \sigma_{x c, t} \eta_{x c, t+1}}_{\text {long-run consumption risk }} \underbrace{-\lambda_{x \pi} \sigma_{x \pi, t} \eta_{x \pi, t+1}}_{\text {long-run inflation risk }} \\
& \underbrace{-\sum_{i \in\left\{\sigma_{c}, \sigma_{\pi}, \sigma_{x c}, \sigma_{x \pi}\right\}} \lambda_{i} \sigma_{\omega_{i}} \omega_{i, t+1}}_{\text {Volatility risk }}
\end{aligned}
$$

which determine the sources and compensation for risks. The $\lambda \mathrm{s}$ denote the equilibrium market prices of risk and are equal to

$$
\begin{gathered}
\lambda_{c}=\gamma, \quad \lambda_{\pi}=0, \quad \lambda_{\lambda}=\frac{\kappa_{1} \rho_{\lambda}-\theta}{1-\kappa_{1} \rho_{\lambda}}, \quad \lambda_{x c}=\frac{\left(\gamma-\frac{1}{\psi}\right) \kappa_{1}}{1-\kappa_{1} \rho_{c c}}, \quad \lambda_{x \pi}=\rho_{c \pi} \frac{\left(\gamma-\frac{1}{\psi}\right) \kappa_{1}^{2}}{\left(1-\kappa_{1} \rho_{c c}\right)\left(1-\kappa_{1} \rho_{\pi \pi}\right)}, \\
\lambda_{\sigma_{c}}=\frac{\left(\gamma-\frac{1}{\psi}\right)(1-\gamma) \kappa_{1}}{2\left(1-\kappa_{1} \nu_{c}\right)}, \quad \lambda_{\sigma_{\pi}}=0, \quad \lambda_{\sigma_{x c}}=\frac{\left(\gamma-\frac{1}{\psi}\right)(1-\gamma) \kappa_{1}^{3}}{2\left(1-\kappa_{1} \rho_{c c}\right)^{2}\left(1-\kappa_{1} \nu_{x c}\right)}, \quad \lambda_{\sigma_{x \pi}}=\rho_{c \pi}^{2} \frac{\left(\gamma-\frac{1}{\psi}\right)(1-\gamma) \kappa_{1}^{5}}{2\left(1-\kappa_{1} \rho_{c c}\right)^{2}\left(1-\kappa_{1} \rho_{\pi \pi}\right)^{2}\left(1-\kappa_{1} \nu_{x \pi}\right)} .
\end{gathered}
$$

Note that the market price of short-run consumption risk, $\lambda_{c}$, is equal to the coefficient of relative risk aversion. Since current inflation is independent from the real economy, the prices of risk associated with it $\left(\lambda_{\pi}, \lambda_{\sigma_{\pi}}\right)$ are zero. In addition, when agents have preference for early resolution of uncertainty $\left(\gamma>\frac{1}{\psi}\right)$, the market price of expected consumption growth risk, $\lambda_{x c}$, is positive and $\lambda_{x \pi}$ is negative whenever expected inflation is bad news for the real economy $\left(\rho_{c \pi}<0\right)$. Furthermore, the market prices of short-, $\lambda_{\sigma_{c}}$, and long-run, $\lambda_{\sigma_{x c}}$, real uncertainties are negative, as well as the market price of the long-run inflation volatility, $\lambda_{\sigma_{x \pi}}$. The price of the time preference shocks risk, $\lambda_{\lambda}$, is guaranteed to be positive whenever $\theta \leq 0$ provided that $\rho_{\lambda}>0$. Note that when $\gamma=\frac{1}{\psi}$ all

\footnotetext{
${ }^{5}$ It is important to note that in the estimation the process for the volatilities is log-normal and given in equation 44. The approximation serves only to obtain an analytical solution for bond prices, which facilitates the state-space representation and estimation of the model. For a detail discussion of these two approximations see Schorfheide et al. (2017).
} 
prices with the exception of $\lambda_{c}$ and $\lambda_{\lambda}$ are zero. Finally, the nominal market prices of risk are all equal to their real counterparts except for $\lambda_{\pi}^{\$}$, which now equals one.

We proceed to price the equilibrium nominal bond yields, while Appendix A.2 provides a solution to $p c_{t}$ and shows an analytical expressions for the equilibrium nominal SDF in terms of the state variables of the economy.

2.3.1 Equilibrium nominal bond yields. Let $P_{t, n}^{\$}$ be the price at time $t$ of an $n$-period zerocoupon nominal bond that pays one unit of the numeraire in $n$ periods. Using the Euler equation, the price can be written recursively as

$$
P_{t, n}^{\$}=E_{t}\left[M_{t+1}^{\$} P_{t+1, n-1}^{\$}\right]
$$

and can be solved by conjecturing that the logarithm of the nominal bond yields, $y_{t, n}^{\$}=-\frac{1}{n} \log P_{t, n}^{\$}$, is linear in the state variables:

$$
y_{t, n}^{\$}=\frac{1}{n}\left(\left(B_{0, n}^{\$}+B_{c, n}^{\$} x_{c, t}+B_{\pi, n}^{\$} x_{\pi, t}+B_{\lambda, n}^{\$} x_{\lambda, t}+B_{\sigma_{c}, n}^{\$} \sigma_{c, t}^{2}+B_{\sigma_{\pi}, n}^{\$} \sigma_{\pi, t}^{2}+B_{\sigma_{x c}, n}^{\$} \sigma_{x c, t}^{2}+B_{\sigma_{x \pi}, n}^{\$} \sigma_{x \pi, t}^{2}\right) .\right.
$$

In Appendix A.4 we provide an analytical expression for the $B$ s, which measure the sensitivity of bond prices to systemic risks. In order to develop economic intuition in terms of the size and sign of each of these coefficients, Figure 1 shows the equilibrium nominal bond yield loadings (evaluated at the posterior median estimates) as a function of bond maturity $n$.

\section{[Place Figure 1 about here]}

Nominal bonds hedge expected consumption risk. A higher expected consumption growth today decreases bond prices and increases nominal yields $\left(B_{c, n}^{\$}>0\right)$, due to the agent's motive to smooth consumption intertemporally. In this scenario, the agent wants to borrow to increase today's consumption and since bonds are in zero net supply, aggregate borrowing cannot increase. In equilibrium, returns on bonds must increase to induce the agent to borrow less. Alternatively, with preference for early resolution of uncertainty $\left(\gamma>\frac{1}{\psi}\right)$, shocks to expected consumption growth are priced. An increase in real volatility increases the agent's uncertainty about future growth and as a result wants to hedge by buying risk-free assets, and in equilibrium, bond yields fall $\left(B_{\sigma_{c}, n}^{\$}, B_{\sigma_{x c}, n}^{\$}<0\right)$. Since shocks to expected consumption growth have larger long-term effects, the 
sensitivity of bond prices to this risk is an order of magnitude larger (in absolute value) than the beta of short-run real volatility risk (see the straight black line in panels (b) and (c) of Figure 1). Furthermore, the longer the bond maturity, the higher the insurance the bond provides against these types of risks and hence the higher its price. This mechanism generates a downward-sloping nominal yield curve. In contrast, the nominal and preference risk channels counteract this mechanism and generate an upward-sloping yield curve.

To understand the nominal channel as described by Bansal and Shaliastovich (2013) and Eraker, Shaliastovich, and Wang (2016), it is useful to consider the following Fisher-type equation:

$$
y_{t}^{\$,(n)}=y_{t}^{(n)}+\frac{1}{n} E_{t} \pi_{t \rightarrow t+n}-\frac{1}{2} \frac{1}{n} \operatorname{Var}_{t} \pi_{t \rightarrow t+n}+\frac{1}{n} \operatorname{Cov}_{t}\left(m_{t \rightarrow t+n}, \pi_{t \rightarrow t+n}\right),
$$

The expected inflation term, $E_{t} \pi_{t \rightarrow t+n}$, directly impacts the valuation of nominal bond prices; nominal yields rise with positive shocks to expected inflation $\left(B_{\pi, n}^{\$}>0\right)$. In addition, if expected inflation reduces expected consumption growth $\left(\rho_{c \pi}<0\right)$, times of high long-term inflation rates are times of low consumption growth. Hence, the covariance term in the above equation is positive and increases with maturity. This term captures the inflation premium in the economy. With preference for early resolution of uncertainty, shocks to expected inflation are also priced, which makes longterm bonds particular risky since shocks that produce a persistent increase in this component generate persistently low real yields and low expected consumption growth. This mechanism is crucial for generating an upward-sloping nominal yield curve.

In contrast to Bansal and Shaliastovich (2013), in this model we include an extra state variable determined by the time preference shocks. As shown in panel (a) of Figure 1, nominal yields are decreasing functions of $x_{\lambda, t}$. When $x_{\lambda, t}$ rises, the representative household values tomorrow's consumption more, relative to the present, and wants to increase savings. In equilibrium, bond prices have to rise $\left(B_{\lambda, n}^{\$}<0\right)$ to clear bond markets. This model is also able to generate an upwardsloping nominal yield curve (note that $B_{\lambda, m}^{\$}>B_{\lambda, n}^{\$}$ if $m>n$ ) via the time preference shocks risk, given that longer maturity bonds have a higher exposure to it. This implies that the model does not rely entirely on the expected inflation channel to reproduce this feature of the data. Furthermore, time preference shocks are a promising mechanism to drive volatile and persistent fluctuations in expected short-term real rates which in turn might generate sizable variations in nominal yield shocks without requiring large fluctuations in expected inflation news. We turn to this issue next. 
2.3.2 Inflation variance ratios. Following Campbell and Ammer (1993) and Duffee (2016), we decompose an $n$-period yield into expectations of average ex-ante real rates, future average inflation, and average expected excess returns over the life of the bond:

$$
y_{t, n}^{\$}=\frac{1}{n} \sum_{i=1}^{n} E_{t} r_{t+i-1,1}+\frac{1}{n} \sum_{i=1}^{n} E_{t} \pi_{t+i}+\frac{1}{n} \sum_{i=1}^{n} E_{t} r x_{r \rightarrow t+1, n-i+1}^{\$},
$$

where the ex-ante real rate is defined as the yield on a one period nominal bond minus expected inflation $r_{t}=y_{t, 1}^{\$}-E_{t} \pi_{t+1}$. The last term on the right is often described as the bond's nominal term premium, which we write as $t p_{t, n}^{\$}$.

Innovations to the $n$-maturity yield from $t-1$ to $t$ are equal to the sum of news about these three components:

$$
\epsilon_{y^{\S}, t}^{(n)}=\epsilon_{r, t}^{(n)}+\epsilon_{\pi, t}^{(n)}+\epsilon_{t p^{\Phi, t}}^{(n)}
$$

where the shocks are defined as

$$
\begin{aligned}
& \epsilon_{y^{\S}, t}^{(n)}=E_{t} y_{t, n}^{\$}-E_{t-1} y_{t, n}^{\$}, \quad \epsilon_{r, t}^{(n)}=\frac{1}{n} \sum_{i=1}^{n} E_{t} r_{t+i-1,1}-\frac{1}{n} \sum_{i=1}^{n} E_{t-1} r_{t+i-1,1} . \\
& \epsilon_{t p^{\$, t}}^{(n)}=E_{t} t p_{t, n}^{\$}-E_{t-1} t p_{t, n}^{\$}, \quad \epsilon_{\pi, t}^{(n)}=\frac{1}{n} \sum_{i=1}^{n} E_{t} \pi_{t+i}-\frac{1}{n} \sum_{i=1}^{n} E_{t-1} \pi_{t+i} .
\end{aligned}
$$

Appendix A.7 provides an analytical expression for these shocks in terms of model parameters. Duffee (2016) defines the unconditional inflation variance ratio of an $n$-period nominal bond as the ratio of variance of inflation news to the variance of yield shocks:

$$
\text { inflation variance ratio } \equiv \frac{\operatorname{Var}\left(\epsilon_{\pi, t}^{(n)}\right)}{\operatorname{Var}\left(\epsilon_{y^{\S}, t}^{(n)}\right)},
$$

and measures the size of expected inflation shocks relative to nominal yield shocks. As shown by Duffee (2016), at quarterly frequency, variances of news about expected inflation account for around 20 percent of variances of yield shocks. This percentage is strongly at odds with standard macro-finance model-implied ratios which often exceed 100 percent. The main problem is that these models do not allow for a large real-rate or excess return channel to generate variations in yield shocks.

This model generates variations in shocks to real rates via four components. To see this it is 
useful to explicitly write the model-implied expressions for $\epsilon_{r, t}^{(n)}$ as

$$
\begin{aligned}
\epsilon_{r, t}^{(n)} & =\frac{1}{n} \frac{1}{\psi} \frac{1-\rho_{c c}^{n}}{1-\rho_{c c}} \sigma_{x c, t-1} \eta_{x c, t}+\frac{1}{n} \frac{\rho_{c \pi}}{1-\rho_{c c}} \frac{1}{\psi}\left[\left(1-\rho_{c c}^{n-1}\right)+\rho_{\pi \pi}\left(1-\rho_{c c}^{n-2}\right)+\cdots+\rho_{\pi \pi}^{n-2}\left(1-\rho_{c c}\right)\right] \sigma_{x \pi, t-1} \eta_{x \pi, t} \\
& +\frac{1}{n} \sum_{i \in\{c, \pi, x c, x \pi\}} B_{\sigma_{i}, 1}^{\$} \frac{1-\nu_{i}^{n}}{1-\nu_{i}} \sigma_{\omega_{i}} \omega_{i, t}-\frac{1}{n} \rho_{\lambda} \frac{1-\rho_{\lambda}^{n}}{1-\rho_{\lambda}} \sigma_{\lambda} \eta_{\lambda, t}
\end{aligned}
$$

The first component is related to persistent movements in expected consumption growth. If $\sigma_{x c, t-1}$ is small and the intertemporal elasticity of substitution, $\psi$, is high, then short-term real rates do not vary much via this component. The second component is associated with the nonneutral effect of expected inflation on expected consumption growth. Bad news for expected inflation translates into bad news for expected consumption growth, which in turn lowers expected future real rates. Hence, variations in nominal yields via this component are mitigated by the negative covariation that it shares with expected inflation shocks, $\epsilon_{\pi, t}^{(n)}$, provided that $\rho_{c \pi}<0$ (i.e., $\operatorname{cov}\left(\epsilon_{r, t}^{(n)}, \epsilon_{\pi, t}^{(n)}\right)<0$ ). In some models, this negative covariance is able to decrease the volatility of nominal yield shocks below the volatility of inflation news, which generates inflation variance ratios above 100 percent. The third component comes from shocks to the volatility processes and affects shocks to real rates through the precautionary savings channel. However, since $\sigma_{\omega_{i}}$ are usually small, they do not contribute much to variations in nominal yield shocks. Finally, the last component is associated with innovations to the time preference shocks. Innovations to $x_{\lambda}$ affect the agents' decisions towards saving, which in turn move short-term real rates. If these innovations are volatile and persistent, then they will generate similar movements in real rates. Section 4.2 shows that this is an important mechanism to match the observed volatility of yield shocks without requiring a counterfactually high volatility of inflation expectations innovations. It also highlights that there is a clear trade-off between matching the inflation variance ratios and other bond market features such as the mean, standard deviation and slope of the nominal interest.

2.3.3 Expected excess bond returns. The presence of stochastic volatility gives rise to time variations in bond risk premia. To see this, consider the one-period excess log return on buying an $n$-period bond at time $t$ and selling it at time $t+1$ as an $n-1$ period bond:

$$
r x_{t \rightarrow t+1, n}^{\$}=n y_{t, n}^{\$}-(n-1) y_{t+1, n-1}^{\$}-y_{t, 1}^{\$}
$$


The one-period expected excess return on nominal bonds is determined by the negative of the covariance between the innovations to excess log returns and the innovations to the nominal SDF:

$$
\begin{aligned}
E_{t} r x_{r \rightarrow t+1, n}^{\$}+\frac{1}{2} V_{t} r x_{r \rightarrow t+1, n}^{\$} & =-\operatorname{cov}_{t}\left(m_{t+1}^{\$}, r x_{r \rightarrow t+1, n}^{\$}\right) \\
& =\underbrace{-\sum_{i \in\left\{\sigma_{c}, \sigma_{\pi}, \sigma_{x c}, \sigma_{x \pi}\right\}} B_{i, n-1}^{\$} \lambda_{i} \sigma_{\omega_{i}}^{2}}_{\text {Volatility risk }} \underbrace{-B_{\lambda, n-1}^{\$} \lambda_{\lambda} \sigma_{\lambda}^{2}}_{\text {Preference risk }} \underbrace{-B_{c, n-1}^{\$} \lambda_{x c} \sigma_{x c, t}^{2}}_{\text {long-run growth risk }} \underbrace{-B_{\pi, n-1}^{\$} \lambda_{x \pi} \sigma_{x \pi, t}^{2}}_{\text {long-run inflation risk }}
\end{aligned}
$$

Hence, the one-period conditional excess return on nominal bonds can be attributed to (i) short- and long-run volatility risks, (ii) preference risk, iii) long-run growth risk, and (iv) long-run inflation risk. The bond risk premium is time-varying and driven by compensations for expected consumption and inflation shocks. Note that nominal bond risk premium decreases with real uncertainty $\left(B_{c, n}^{\$}>0\right.$ and $\left.\lambda_{x c}>0\right)$ and increases at times of high inflation uncertainty $\left(B_{\pi, n}^{\$}>0\right.$ and $\left.\lambda_{x \pi}<0\right)$. In addition, long-term bonds also command a constant positive risk premium induced by the time preference shocks risk $\left(B_{\lambda, n-1}^{\$}<0\right.$ and $\left.\lambda_{\lambda}>0\right)$.

2.3.4 Term premium. Solving $t p_{t, n}^{\$}$ in equation 12 delivers an expression for the $n$-maturity nominal bond term premium:

$$
t p_{t, n}^{\$}=\mathrm{const}+\frac{1}{n}\left(B_{\sigma_{x c}, n}^{\$}-B_{\sigma_{x c}, 1}^{\$} \frac{1-\nu_{x c}^{n}}{1-\nu_{x c}}\right) \sigma_{x c, t}^{2}+\frac{1}{n}\left(B_{\sigma_{x \pi}, n}^{\$}-B_{\sigma_{x \pi}, 1}^{\$} \frac{1-\nu_{x \pi}^{n}}{1-\nu_{x \pi}}\right) \sigma_{x \pi, t}^{2}
$$

The term premium measures the additional compensation a risk averse investor needs to be indifferent between purchasing an $n$-period bond and holding it until maturity or rolling over one-period bonds for a total of $n$ periods. Under the expectation hypothesis the term premium is constant. In this model, the term premium is time-varying due to variations in bond risk premia driven by long-run real and nominal uncertainties. In Section 4.4 we show that the term premium implied by the model is able to account for the evidence of bond return predictability documented in Cochrane and Piazzesi (2005) and for the failure of the expectation hypothesis, as identified in Campbell and Shiller (1991). Furthermore, in Section 4.6, we show that the model is able to generate sizable variations in term premium. Finally, note that from this expression it is straight forward to compute 
the term premium shocks component, $\epsilon_{t p^{\Phi, t}}^{(n)}$, given by

$$
\epsilon_{t p^{\Phi}, t}^{(n)}=\frac{1}{n} \sum_{i \in\{x c, x \pi\}}\left(B_{\sigma_{i}, n}^{\$}-B_{\sigma_{i}, 1}^{\$} \frac{1-\nu_{i}^{n}}{1-\nu_{i}}\right) \sigma_{\omega_{i}} \omega_{i, t} .
$$

This channel cannot contribute much to the variance of yield shocks, since, as described before, $\sigma_{\omega_{i}}$ is usually small. Moreover, note that time preference shocks do not contribute to the term premium given the assumption of homoskedastic innovations in agents' rate of preferences.

\subsection{State-space representation of the model}

The state-space representation of the model consists of two equations. The first is the measurement equation, which links the observed variables with the model-implied ones and adds measurement errors to capture discrepancies that might be present between these two components. In addition, measurement errors account for the stochastic singularity problem that arises when the number of observables is greater than the number of state variables. The second is the state transition equation, which describes the law of motion of the state variables.

2.4.1 Measurement equation. To distinguish observed variables from model-implied ones, we use the superscript $o$. The observables are consumption growth, $\Delta c^{o}$, inflation, $\pi^{o}$, and zero-coupon nominal bond yields, $y^{\$, o}$.

Measurement equation for consumption growth. To aid the identification of time-varying volatilities, we use the highest frequency available which is monthly (e.g., Drost and Nijman 1993). However, monthly consumption data has nontrivial measurement errors masking the actual dynamics of consumption (e.g., Wilcox 1992). Following Schorfheide et al. (2017), we include monthly measurement errors in the process of consumption that average out at the quarterly frequency and which allow the identification of the persistent component of consumption growth as well as time-varying short- and long-run volatilities.

Let the subscript $t$ represent the monthly time as $t=3(j-1)+m$, where $m$ indexes the 
month within quarter $j$ and $m=1,2,3$. Specifically, we assume that:

$$
\begin{aligned}
\Delta c_{3(j-1)+1}^{o}= & \Delta c_{3(j-1)+1}+\sigma_{\epsilon}\left(\epsilon_{3(j-1)+1}-\epsilon_{3(j-2)+3}\right) \\
& -\frac{1}{3} \sum_{m=1}^{3} \sigma_{\epsilon}\left(\epsilon_{3(j-1)+m}-\epsilon_{3(j-2)+m}\right)+\sigma_{\epsilon}^{q}\left(\epsilon_{(j)}^{q}-\epsilon_{(j-1)}^{q}\right) \\
\Delta c_{3(j-1)+m}^{o}= & \Delta c_{3(j-1)+m}+\sigma_{\epsilon}\left(\epsilon_{3(j-1)+m}-\epsilon_{3(j-2)+m-1}\right) \text { for } m=2,3 \text { and } \epsilon, \epsilon^{q} \sim N(0,1) .
\end{aligned}
$$

$\sigma_{\epsilon}$ and $\sigma_{\epsilon}^{q}$ denote the standard deviation of monthly and quarterly consumption measurement errors. Note that under this specification, aggregating the monthly consumption series to the quarterly frequency according to

$$
\Delta c_{j}^{q, o}=c_{j}^{q, o}-c_{j-1}^{q, o}=\sum_{\tau=1}^{5} \frac{3-|\tau-3|}{3} \Delta c_{3 j-\tau+1}^{o}
$$

averages out the monthly measurement errors; $\Delta c_{j}^{q, o}=\Delta c_{j}^{q}+\sigma_{\epsilon}^{q}\left(\epsilon_{(j)}^{q}-\epsilon_{(j-1)}^{q}\right)$. Under this specification, the levels of monthly consumption are constructed by distributing quarterly consumption over the three months of a quarter; this distribution is based on a noisy monthly proxy series. Furthermore, monthly consumption growth rates are proportional to the growth rates of the proxy series and monthly consumption adds up to quarterly consumption. At quarterly frequency, consumption growth is strongly positively autocorrelated, while, at the monthly frequency, it exhibits a significant negative autocorrelation, which provides evidence for a negative moving average component. The assumed measurement error model for consumption is able to reconcile these features.

Measurement equation for inflation. We assume that inflation is measured without any errors and define

$$
\pi_{t+1}^{o}=\pi_{t+1}
$$

because, at the estimation stage, there was no clear way to disentangle the measurement errors from the transitory iid shocks.

Measurement equation for Nominal bond yields. We assume that nominal bond yields are contaminated by iid measurement errors and define

$$
y_{t+1, n}^{\$, o}=y_{t+1, n}^{\$}+\sigma_{y_{n}} \epsilon_{y_{n}, t+1}, \quad \text { with } \quad \epsilon_{y_{n}, t+1} \sim N(0,1)
$$


where $\sigma_{y_{n}}$ denotes the standard deviation of the yields measurement error. Assuming small yield measurement errors is not a strong modeling assumption, since zero-coupon Treasury yields are not directly observed for long maturities and have to be bootstrapped from observed bond prices.

Altogether, the measurement equation of the state-space representation stacks each particular measurement equation and is given by:

$$
Y_{t+1}^{o}=A_{t+1}\left(D+Z s_{t+1}+Z^{v} s_{t+1}^{v}\left(h_{t+1}\right)+\Sigma^{u} u_{t+1}\right), \quad \text { with } \quad u_{t+1} \sim N(0, I)
$$

where the vector of observables $Y_{t+1}^{o}$ contains consumption growth, $\Delta c^{o}$, inflation, $\pi^{o}$, and nominal bond yields, $y^{\$, o}$. The assumed measurement errors and the model solution provide the link between $Y_{t+1}^{o}$ and the vectors of state variables $s_{t+1}$ and $s_{t+1}^{v}\left(h_{t+1}\right)$. Broadly, $s_{t+1}$ includes the predictable components of consumption growth, $x_{c, t}$, and inflation, $x_{\pi, t}$, as well as the time preference shocks, $x_{\lambda, t}$, innovations to fundamentals, and the measurement errors of consumption. The vector $s_{t+1}^{v}\left(h_{t+1}\right)$ is a function of the $\log$ volatilities $h_{t+1}=\left[h_{x c, t+1}, h_{x \pi, t+1}, h_{c, t+1}, h_{\pi, t+1}\right]^{\prime}$ and stacks the short-, and long-run real and nominal uncertainties. The vector $u_{t+1}$ consists of bondspecific measurement errors where the diagonal of $\Sigma^{u}$ contains the standard deviation of the yields measurement errors, $\sigma_{y_{n}}$. Finally, $A_{t+1}$ is a selection matrix that accounts for changes in the data availability induced by the specification of the measurement error model of consumption growth.

2.4.2 State transition equation. The state transition equation provides the dynamics of the state variables broadly given by the assumed process of the time preference shocks in (2) and by the predictable component of consumption growth and inflation in (3). It can be written as:

$$
s_{t+1}=\Phi s_{t}+v_{t+1}\left(h_{t}\right), \quad \text { and } \quad h_{t+1}=\Psi h_{t}+\Sigma_{h} \omega_{t+1} \quad \text { with } \quad \omega_{t+1} \sim N(0, I)
$$

where the variance of $v_{t+1}\left(h_{t}\right)$ depends on the log volatilities at time $t$. In Appendix B, we describe each particular component of the state-space representation in detail. We now proceed to describe the estimation procedure and results. 


\section{Estimation}

In Section 3.1 and 3.2 , we present the estimation procedure and the data set used. In Section 3.3 . we analyze the prior and posterior distribution of the model parameters, while in Section 3.4 , we describe the smoothed latent state estimates.

\subsection{Estimation procedure}

We use a Metropolis-Hastings sampler for posterior inference. We make inference on two different sets of parameters. One set corresponds to the assumed process for the macroeconomic dynamics denoted by:

$$
\begin{gathered}
\Theta_{\text {macro }}=\left\{\Theta_{c \pi}, \Theta_{h}\right\} \quad \text { with } \\
\Theta_{c \pi}=\left\{\mu_{c}, \sigma, \varphi_{x c}, \rho_{c c}, \rho_{c \pi}, \mu_{\pi}, \varphi_{\pi}, \varphi_{x \pi}, \rho_{\pi \pi}, \sigma_{\epsilon}, \sigma_{\epsilon}^{q}\right\}, \quad \text { and } \quad \Theta_{h}=\left\{\rho_{h_{c}}, \sigma_{h_{c}}, \rho_{h_{\pi}}, \sigma_{h_{\pi}}, \rho_{h_{x c}}, \sigma_{h_{x c}}, \rho_{h_{x \pi}}, \sigma_{h_{x \pi}}\right\} .
\end{gathered}
$$

The second set of parameters is related to the preference of the representative household:

$$
\Theta_{\text {pref }}=\left\{\delta, \psi, \gamma, \rho_{\lambda}, \sigma_{\lambda}, \sigma_{y^{n}}\right\}
$$

We include the yields measurement errors in this second set, and define the union of $\Theta_{\text {macro }}$ and $\Theta_{\text {pref }}$ as follows:

$$
\Theta=\left\{\Theta_{\text {macro }}, \Theta_{\text {pref }}\right\}
$$

It is important to differentiate these two sets of parameters since we estimate $\Theta_{\text {macro }}$ with and without considering bond prices in the estimation. If $\Theta_{\text {macro }}$ does not change much between these two different informational contends, this suggests that there is enough information in the macroeconomic variables to price the nominal yield curve. However, if the difference in parameter estimates is large, the estimation might be achieving a better fit for bond yields while sacrificing the fit of consumption growth and inflation.

To make Bayesian inference about $\Theta$ and the latent state vectors, we adopt the econometric approach proposed by Schorfheide et al. (2017). We specify a prior distribution $p(\Theta)$ and update our a priori beliefs about the parameter vector $\Theta$, in view of the sample information $Y^{o}$. The state of knowledge regarding $\Theta$ is summarized by the posterior distribution $p(\Theta \mid Y)$ and Bayes Theorem 
provides the formal link:

$$
p\left(\Theta \mid Y^{o}\right)=\frac{p\left(Y^{o} \mid \Theta\right) p(\Theta)}{p\left(Y^{o}\right)} .
$$

Given the presence of nonlinearities induced by the volatility states it is not possible to directly evaluate the likelihood function, $p\left(Y^{o} \mid \Theta\right)$, via the Kalman filter. Instead, we use a sequential Monte Carlo filter to approximate $p\left(Y^{o} \mid \Theta\right)$. In essence, we represent the distribution of volatilities using a swarm of particles; conditional on these particles, the model has a linear and Gaussian state-space representation that allows us to apply the Kalman filter to equation 222 and 23). Once we are able to draw from the posterior distribution $p\left(\Theta \mid Y^{o}\right)$, we use a random-walk Metropolis-Hasting algorithm to draw the parameter vector $\left\{\Theta^{(s)}\right\}_{s=1}^{N_{s i m}}$ as in Fernández-Villaverde and Rubio-Ramírez (2007) and Andrieu, Doucet, and Holenstein (2010). Detailed steps of the algorithm are available in Appendix B.3 and for further reference regarding the implementation of the particle filter see Herbst and Schorfheide (2015).

\subsection{Data}

The data is standard in the literature and consists of monthly observations of real per-capita consumption growth, inflation, and nominal bond yields with maturities of between one and five years. The sample period is determined by the availability of the consumption growth series and spans from 1960:M1 to 2014:M12 ${ }^{6}$ Specifically, we use the per-capita series of real consumption expenditure on nondurables and services from the National Income and Product Account published by the Bureau of Economic Analysis. We downloaded CPI inflation from the Federal Reserve Bank of St. Louis. We constructed growth rates for consumption and inflation by computing the differences of the log series. Lastly, monthly nominal bond yields were obtained from the CRSP Fama-Bliss zero coupon bond files.

\subsection{Prior and posterior distribution}

We will first describe our choice of priors and then we proceed to analyze the posterior estimates.

\footnotetext{
${ }^{6}$ Monthly consumption growth starts in 1959:M2. However, to have non-missing values for all years we decided to start in 1960:M1.
} 
3.3.1 Prior distribution. In general, we attempted to restrict the parameter space to economically plausible values while at the same time be as uninformative as possible. For example, our prior distribution for the persistence parameter of the predictable component of consumption growth cover values that imply a near iid or a unit root process for consumption growth. Similarly, we do not restrict the preference of the representative household to have early, $\theta<0$, or late, $\theta>0$, resolution of uncertainty. The first columns of Table 1 show the assumed prior distribution, as well as the 5 and 95 percentiles, while we relegate to Appendix B.4 a detail discussion of the priors.

\section{[Place Table 1 about here]}

3.3.2 Posterior distribution. The last six columns of Table 1 show the 5, 50, and 95 percentiles of the posterior distribution under two different scenarios. Columns 5 to 7 show the posterior distribution if bond prices are included in the estimation, while columns 8 to 10 only consider the information contained in macro data..$^{7}$ It is important to highlight that the posterior intervals are considerably narrower if bond prices are considered in the estimation, suggesting that bond yields provide additional information that reduces the parameters posterior uncertainty. We will first describe the parameters related to the agent's preference, $\Theta_{\text {pref }}$, and then proceed to analyze the parameter estimates in $\Theta_{\text {macro }}$.

The coefficient of risk aversion, $\gamma$, and the elasticity of substitution, $\psi$, have a posterior median value of around 8.0 and 1.80, respectively. These estimates are consistent with the parameter values highlighted in the long-run risk literature and imply preferences for early resolution of uncertainty. It is also worth noting that the posterior median value of the persistence parameter of the time preference shocks, $x_{\lambda, t}$, is around 0.95. Furthermore, a value of $\varphi_{\lambda} \approx 0.25$ suggests that they are considerable less volatile than consumption growth.

With respect to the parameters associated with the macroeconomic dynamics, $\Theta_{\text {macro }}$, we found that the parameter estimates that incorporate bond prices in the estimation are in close

\footnotetext{
${ }^{7}$ To estimate the model that only includes macro data in the estimation, we cast equation 3 into a state-space form and use Metropolis-within-Gibbs sampler for posterior inference iterating over the three conditional distributions. In Appendix C.3 we provide detailed steps of the Metropolis-within-Gibbs sampler algorithm. We also assume a common stochastic volatility process (i.e., $h_{c, t}=h_{x c, t}$ and $h_{\pi, t}=h_{x \pi, t}$ ). Allowing for four different stochastic volatilities, the 90 percent confidence interval increased considerably for most of the parameters and the log marginal data density decreased from 6055 to 5761 . With these evidence, we conclude that there seems not to be enough information in consumption growth and inflation to reliably identify four different stochastic volatilities.
} 
agreement with the ones that only consider macro variables. Some features are worth noting. First, the posterior median estimates of $\left(\rho_{c c}, \rho_{c \pi}, \rho_{\pi \pi}\right)$ are remarkably similar with $(0.96,-0.01,0.99)$ or without $(0.95,-0.02,0.98)$ considering bond prices in the estimation. In all cases, the posterior confidence interval of the latter contains the confidence interval of the former, suggesting that there is enough information in the macro data to identify them (adding the information in bond prices sharpens inference). This result is driven mostly by the measurement error model of consumption and the assumed stochastic volatility processes for consumption growth and inflation. Appendix B.5 shows that accounting for measurement errors in consumption reveals a persistent component in consumption growth: In the absence of measurement errors $\rho_{c c}$ drops to -0.20 (See Table B1). Appendix B.5 also shows that adding stochastic volatility to the process of the macro variables improves the model fit considerably (measured by the log marginal data density) and decreases the posterior uncertainty of the parameter estimates (measured by the 90 percent credible interval). Furthermore, there is enough information in the macro data to identify $\rho_{c \pi}$, which is negative and significant as suggested by the 95 percentile in column 10 of Table 1 . This negative nonneutral effect of inflation on growth is consistent with Piazzesi and Schneider (2007) and Bansal and Shaliastovich (2013), who used bond yields to identify $\rho_{c \pi}$.

In the model, time-variation in term premia is driven by the stochastic volatilities of expected consumption growth and expected inflation. Once bond prices were included in the estimation, the parameters governing the persistence and standard deviation of the long-run volatility processes (i.e., $\left(\rho_{h_{x c}}, \sigma_{h_{x c}}\right)$ and $\left.\left(\rho_{h_{x \pi}}, \sigma_{h_{x \pi}}\right)\right)$ increased somewhat in order to match the sizable variations observed in the term premia dynamics. Starting from an agnostic prior, $\sigma_{h_{x c}}$ and $\sigma_{h_{x \pi}}$ increased from an approximate value of 0.11 and 0.30 to 0.13 and 0.33 , respectively. Similarly, $\rho_{h_{x c}}$ and $\rho_{h_{x \pi}}$ increased from 0.97 and 0.81 to approximately 0.98 and 0.96 , respectively. Alternatively, since the high-frequency movements in consumption growth and inflation are negligible for asset prices but important for tracking the macroeconomic data, we should not expect similar changes in the posterior parameter estimates governing these dynamics 8 Consistent with this idea, the posterior median estimates of $\left(\rho_{h_{c}}, \sigma_{h_{c}}\right)$ and $\left(\rho_{h_{\pi}}, \sigma_{h_{\pi}}\right)$ were quite similar between these two scenarios.

\footnotetext{
${ }^{8}$ By adding short-run conditional heteroskedasticity by allowing for time variation in $\sigma_{c, t}$ and $\sigma_{\pi, t}$ and shutting down the variation in $\sigma_{x c, t}$ and $\sigma_{x \pi, t}$, the log marginal data density increased from 5870 to 6054 , which is almost as high as the value obtained by assuming a common stochastic volatility process (i.e., $h_{c, t}=h_{x c, t}$ and $h_{\pi, t}=h_{x \pi, t}$ ) equal to 6055 . Hence, we conclude that short-run conditional heteroskedasticity improves considerably the model fit of the assumed process of the macro variables.
} 
Finally, the scale variance parameters associated with consumption growth and inflation decreased in order to counteract the increased variation of the predictable components of consumption growth and inflation: The posterior median of $\varphi_{x c}, \varphi_{x \pi}$, and $\varphi_{\pi}$, decreased from $0.22,0.22$, and 0.98 to $0.19,0.1$, and 0.98 , respectively. Table 2 illustrates this point by showing the 5 , 50, and 95 percentiles of the implied unconditional mean of the stochastic volatilities, $E\left[\sigma_{i, t}\right]$, which is a function of $\rho_{h_{i}}, \sigma_{h_{i}}$, and $\varphi_{i}$, and can be written as:

$$
E\left[\sigma_{i, t}\right]=\varphi_{i} \sigma_{c} \exp \left(0.5 \frac{\sigma_{h_{i}}^{2}}{1-\rho_{h_{i}}^{2}}\right)
$$

For instance, note that although the estimates of $\rho_{h_{x c}}$ and $\sigma_{h_{x c}}$ increased once we consider bond prices in the estimation to better capture the dynamics of the term premium, $\varphi_{x c}$, decreased enough such that the unconditional mean of the process of $\sigma_{x c, t}$ remained remarkable similar to the estimates that do not consider the information contained in bond prices: The $90 \%$ credible interval contains the posterior median of $E\left[\sigma_{x c, t}\right]$ from the macro-only estimation. The same holds for the remaining three volatility process.

\section{[Place Table 2 about here]}

Overall, the parameter estimates that incorporate bond prices in the estimation are in close agreement with the ones that only consider macro aggregates. The fact our macro based estimates are similar to those that also incorporate bond yields is important since it demonstrates they are not completely driven by the financial variables 9 Specifically, adding bond prices contributes to smaller credible intervals and allows the identification of four separate volatility components: two drive the dynamics of the predictable components of consumption growth and inflation, and the remaining two govern temporary and independent shocks to the realization of the macro aggregates. The former two are important for bond prices, while the latter ones are important for tracking the macro data. Next we show that the filtered macro state variables follow very closely the dynamics of the observed macro data, while in Section 4 we show the model is able to match a rich set of macro and bond market features.

\footnotetext{
${ }^{9}$ Relative to Creal and $\left.\mathrm{Wu} \sqrt{2016}\right)$ this result is primarily driven by incorporating the measurement error structure and accounting for temporal aggregations. They also primarily focus on the term premium properties while our estimation also successfully targets the failure of the expectation hypothesis, risk premia predictability and inflation variance ratios.
} 


\subsection{Latent state variables}

This section describes the filtered estimates of the latent state variables. In panels (a) and (b) of Figure 2, we show the smoothed expected consumption growth, $x_{c, t}$, and expected inflation, $x_{\pi, t}$, obtained from the estimation that includes bond prices. We also overlay the observed monthly consumption growth and inflation series. In addition, the shaded areas denote the National Bureau of Economic Research (NBER) recession dates. $x_{c, t}$ exhibits declines during each recession, with the largest decline during the recent financial crisis. On the other hand, $x_{\pi, t}$ has its highest point during the Great Inflation period and decreases afterwards. Note that recessions during the 1970s and 1980s were accompanied by an increase in the expected inflation component, and that this behavior suddenly reversed during the last three crises, where $x_{\pi, t}$ remained constant or slightly declined.

\section{[Place Figure 2 about here]}

In panels (c) and (d) of Figure 2, we plot the volatilities of the long-run growth, $h_{x c}$, and inflation rate, $h_{x \pi}$, respectively. Both series vary considerably: $h_{x c}$ starts high and shows a decreasing trend with spikes during the crises of 1975 and 1980. It remained at low levels until the late 1990s, after which it started to increase and has stayed at relatively high levels ever since. $h_{x \pi}$ displays different properties. In the 1960s it was at low levels; it jumped around during the 1970s with peaks between 1980 and 1985 and remained at high values until 1990, when it started to decrease with wide fluctuations around 2000. Similarly, in panels (e) and panel (f), we plot the smoothed series for the high frequency components of consumption growth, $h_{c}$, and inflation, $h_{\pi}$. The first thing to note is that the magnitude of both series is remarkably smaller. Also of note is that $h_{c}$ captures the drop in growth volatility that occurred around the 1980s, also known as the Great Moderation period and it shows a decreasing pattern from that date onwards. On the other hand, $h_{\pi}$ practically spikes at every recession, but periods of an increase in $h_{\pi}$ also occur during expansions.

\section{[Place Figure 3 about here]}

Time preference shocks determine the attitudes of the representative household towards savings and they are, by assumption, orthogonal to the real economy. With this in mind, an interesting 
candidate to correlate the smoothed demand shocks estimates is the adjusted National Financial Conditions Index (adjusted FCI) published by the Federal Reserve of Chicago. This index measures risk, liquidity, and leverage in money, debt, and equity markets, and isolates a component of financial conditions uncorrelated with economic conditions 10 Figure 3 , shows the posterior median of the smoothed time preference shocks along with the negative value of the adjusted FCI divided by a constant to have similar magnitudes ${ }^{11}$ Given these adjustments, a positive value of the adjusted FCI index suggests loose financial markets relative to an historical average. The correlation between these series is remarkably high, with a value of around 50 percent ${ }^{12}$ suggesting that tighter financial conditions today are associated with a lower willingness of the agents to save for future periods.

We assume that the time preference shocks are orthogonal to consumption growth and to its predictable component. In a general equilibrium framework this will not likely be the case, since innovations to the time preference parameter will affect the household decisions towards consumption, saving, and investment, which in turn will affect production outcomes and the expected path of consumption growth. To address this concern, we computed the 90 percent confidence interval of the correlation between innovations to the time preference shocks, $\sigma_{\lambda} \eta_{\lambda, t+1}$, and shocks to consumption growth, $\sigma_{c, t} \eta_{c, t+1}$, and between $\sigma_{\lambda} \eta_{\lambda, t+1}$ and shocks to expected consumption growth, $\sigma_{x c, t} \eta_{x c, t+1}$. These correlations are small, ranging from -0.08 to 0.10 and from 0.01 to 0.15 , respectively. These results are consistent with Schorfheide et al. (2017), who also assume uncorrelated shocks between the preference shocks and the cash flow components and conclude that there is no strong evidence against the assumption of orthogonal preference shocks.

In sum, the latent state variables are directly linked to macroeconomic risk factors or are correlated to measures of risk, liquidity, and leverage in financial markets uncorrelated with economic conditions. In lieu of these findings, we now turn to analyze how well does the model match key features of the term structure.

\footnotetext{
${ }^{10}$ The Adjusted National Financial Conditions Index is provided by the Federal Reserve Bank of Chicago; the series starts in 1973 and can be downloaded at https://alfred.stlouisfed.org/series?seid=ANFCI

${ }^{11}$ In sum, we divided the original adjusted FCI series by -1200 .

${ }^{12}$ This correlation increases to 65 percent if we remove the high frequency components of the series by taking a twelve month moving average.
} 


\section{Asset pricing implications}

In Section 4.1, we analyze how well the model matches standard macro and bond yield moments. In Section 4.2 , we quantify to what extent the volatility of nominal yield shocks is due to the volatility of expected inflation news. In Section 4.3, we analyze the relative contribution of each risk factor to movements in nominal bond yields. In Section 4.4, we study the bond predictability results implied by the model. Sections 4.5 and 4.6 show the relative contribution of risk factors to movements in expected excess bond returns and term premium, respectively.

\subsection{Model Implications for macro and bond yield moments}

While we are targeting the joint distribution of macroeconomic and bond yield variables, it is informative to document how close the model-implied moments are to those observed in the data. Table 3 shows the percentiles of the posterior predictive distribution for several sample moments 13 based on parameters drawn from the posterior distribution that considers bond prices (columns 3 to 5), and from that which only considers macro data (columns 6 to 8). For the latter, we use the posterior medians of the preference parameters $\Theta_{\text {pref }}$. Finally, in the second column, we show the same moments computed from the observed series. All moments are annualized.

\section{[Place Table 3 about here]}

The model does a good job of reproducing the macro moments while at the same time accounting for the level and slope of the nominal yield curve as well as the standard deviation of nominal yields. All of the data moments are well within the 90 percent credible interval. In the results of the estimation that includes bond prices, the model performs well in matching the macroeconomic moments: the model-implied posterior median values are fairly close to their data estimates. Regarding the bond yield moments, the model-implied posterior medians match the decreasing pattern in the standard deviation of bond yields across maturities and at the same time captures the upward-sloping unconditional mean of the nominal term-structure. It also does a good job in accounting for the difference between the long and the short end (i.e., slope) of the yield curve: in

\footnotetext{
${ }^{13}$ To this end, we sampled $M$ draws of $\Theta$ from its posterior distribution and simulated the model for 660 periods, which corresponds to the number of monthly observations in our estimation sample. This gave us $M$ simulated trajectories of $Y^{o}$. For each one of them, we computed several statistics. In the implementation we set $M=10,000$.
} 
the data and in the model it is around 60 and 80 basis points, respectively. In the results of the estimation that does not include bond prices, the model is still able to match these features of the data and generate credible intervals that contain the sample data moments, as shown in the last three columns of Table 3, although the standard deviation of the nominal yields is somewhat lower than the moments obtained by also including bond prices in the estimation.

\subsection{Model implications for inflation variance ratios}

To compute the data-implied inflation variance ratios for long horizons, we need to rely on a statistical model for expected inflation and bond yields. In Section 4.2.1, we extend Duffee's analysis by assuming heteroskedasticity in both inflation news and yield shocks, instead of splitting the estimation in different subperiods, and increase the frequency of the data from quarterly to monthly observations. Accounting for conditional information brings the data and model implied moments in closer agreement. Overall, we show that conditional inflation variance ratios tend to be higher (around 33 percent) than unconditional inflation variance ratios (around 26 percent). Finally, in Section 4.2 .2 , we present the model-implied inflation variance ratios.

4.2.1 Inflation variance ratios: Data. Since survey inflation forecasts are focused at relatively short horizons (usually less than one year), we obtained data estimates of the inflation variance ratios from a statistical model. For consistency, and following Stock and Watson (2007), we extracted oneto five-year inflation forecast innovations, $\epsilon_{\pi, t}^{(n)}$, from the process of inflation specified in equation (3). Following Duffee (2016), we also included information from the surveys of market practitioners that are released at a different (quarterly) frequency. As argued by Ang, Bekaert, and Wei (2007), surveybased inflation forecasts are more accurate than model-based ones, which sharpens the inference about $\epsilon_{\pi, t}^{(n)}$. Specifically, we used the CPI forecast from the Survey of Professional Forecasters (SPF) of one to four quarters ahead. Since this series starts in 1983Q3 and the estimation period of the structural model begins in 1960Q1, we also include one to four quarter ahead SPF forecasts of the Price Index for Gross National Product (PGDP) from 1968Q to 1983Q2 ${ }^{14}$ Hence, the estimation period of the statistical model goes from 1968Q4 to 2014Q14.

\footnotetext{
${ }^{14}$ Although these two different price forecasts are highly autocorrelated (around 0.97), we allow for different measurement errors once we cast the model into the State-space form. Appendix C provides further details.
} 
Similarly, to compute shocks to the $n$-period yield, $\epsilon_{y^{\Phi}, t}^{(n)}$, we follow Duffee 2016) and assume that bond yields follow martingales

$$
\begin{gathered}
y_{t+1}^{(n)}=y_{t}^{(n)}+\sigma_{y^{(n)}, t} \eta_{y^{(n)}, t+1} \quad \text { and } \quad \eta_{y^{(n)}, t+1} \sim i . i . d . N(0,1) \quad \text { where } \\
\sigma_{y^{(n)}, t}=\sigma_{y^{(n)}} \exp \left(h_{y, t}\right), \quad h_{y^{(n)}, t+1}=\rho_{h_{y}^{(n)}} h_{y^{(n)}, t}+\sigma_{h_{y}^{(n)}} \omega_{y^{(n)}, t+1}, \quad \text { and } \quad \omega_{y, t+1} \sim \text { i.i.d.N }(0,1),
\end{gathered}
$$

where $\epsilon_{y^{\S}, t}^{(n)}=\sigma_{y^{(n)}, t} \eta_{y^{(n)}, t+1}$. For bond yields, the martingale assumption generates lower forecast errors than both, survey based (e.g., Cieslak 2016) and parametrized models (e.g., Duffee 2002). We also allow for bond yield shocks, $\eta_{y^{(n)}, t+1}$, and expected inflation shocks, $\eta_{x \pi, t+1}$, to be correlated. Finally, given an estimate of $\epsilon_{\pi, t}^{(n)}$ and $\epsilon_{y^{\S}, t}^{(n)}$, it is straightforward to compute the inflation variance ratios. In Appendix C, we provide details with respect to the estimation procedure.

\section{[Place Table 4 about here]}

The last three columns of Table 4 shows the inflation variance ratios along with the 90 percent confidence interval for maturities of one to five years. For the sake of completeness, we also report the estimates based on the homoskedastic version of the model and, in Appendix C.3. we provide the parameter estimates together with the assumed prior distribution. In the case of homoskedastic innovations, the posterior medians of the unconditional inflation variance ratios for all bond maturities were around 26 percent whereas the 95 percentiles were below 37 percent. These results are by and large consistent with the estimates reported by Duffee (2016), and can be viewed as a corroboration of his findings. Nevertheless, a careful inspection of the innovations suggests that these vary through time and exhibit heteroskedasticity. Therefore, instead of doing subperiod analysis, in the last three columns of Table 4 we report the estimates of the model that allows for stochastic volatility. Allowing for heteroskedasticity in inflation shocks and innovations to bond yields improves the model fit significantly, as evidenced by the log marginal data density reported at the end of Table C1. It also shifts the distribution of the unconditional inflation variance ratios towards higher values: the posterior medians were around 32 percent for a one-year bond and 34 percent for a five-year bond. However, the 95 percentiles were all below 62 percent. These ratios are still at odds with corresponding values from standard macro-finance term structure models 15

\footnotetext{
${ }^{15}$ The inflation variance ratios are expected to increase under the stochastic volatility case due to a Jensen's term,
} 
Robustness. As a robustness check on our findings, we repeated the same exercise for a quarterly version of the statistical model considering different measures of inflation expectations reported by SPF. In Appendix C.4 we report the estimates in detail, while briefly describing the results here. Overall, for all bond maturities considered, expected inflation news does not contribute much to the variation of bond innovations. The posterior medians were around 12 percent. Allowing for stochastic volatility almost doubles the inflation variance ratios relative to the homoskedastic case, improves the model fit; furthermore, the 95 percentile of the inflation variance ratios for all SFP measures considered are below 50 percent.

\subsubsection{Inflation variance ratios: Model-implied. In panel (a) through (e) of Figure 4. we} plot the probability density distribution of the predictive distribution of the model-implied inflation variance ratios for the one to five year bond, respectively ${ }^{16}$ The dark shaded density is based on the macro-finance model that includes time preference shocks (Model w/ TPS), while the light shaded density does not include them (Model w/o TPS) ${ }^{17}$ The straight and dashed black lines denote the posterior median inflation variance ratios from the statistical model that assumes homoskedastic (Data w/o SV) and heteroskedastic shocks (Data w/SV) also reported in Table 4.

\section{[Place Figure 4 about here]}

The model-implied inflation variance ratios from the model that assumes time preference shocks are in close agreement with the data estimates. Based on this evidence, we conclude that the model does not resort to an expected inflation channel that counterfactually dominates the variation in nominal yield shocks. Interestingly, if we turn the time preference shocks channel off, the model provided that the correlation between $\operatorname{Var}_{t}\left(\epsilon_{\pi, t}^{(n)}\right)$ and $\operatorname{Var}_{t}\left(\epsilon_{y^{\$}, t}^{(n)}\right)$ is not too high. To see this, note that

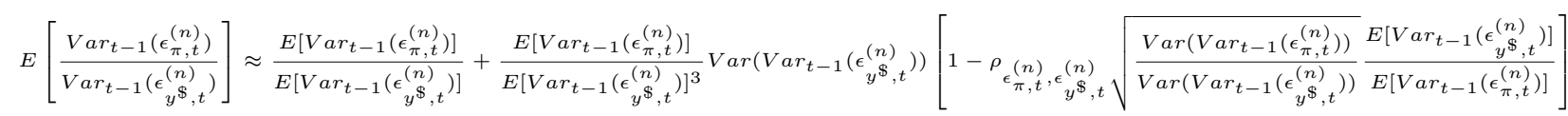

where $\rho_{\epsilon_{\pi, t}^{(n)}, \epsilon_{y^{\mathbb{S}, t}}^{(n)}}$ denotes the correlation between the corresponding variance terms. The first term on the right hand side corresponds to the inflation variance ratios under homoskedastic shocks.

${ }^{16}$ Specifically, we sample $M$ draws of $\Theta$ from its posterior distribution and simulate the models for 660 periods, which corresponds to the number of monthly observations in the estimation sample. For each one of these simulated trajectories and for each time period $t$ we computed the implied inflation variance ratio and took the average across time for a total of $M$ inflation variance ratios. We set $M=10,000$.

${ }^{17}$ To this end, we solved the model without time preference shocks, cast it into the state-space form, and estimated it under the same estimation procedure. We made sure that the model estimation matched the same data moments and predictability results as the model that assumes time preference shocks. Results available upon request. 
is not able to match this moment of the data. Note that the probability density distributions shift to the right (light shaded area) to inflation variance ratios values centered approximately around 80 percent. Furthermore, the posterior median population values also increased from 17 and 39 percent to 69 and 86 percent for the one and five year bond, respectively. These values are somewhat smaller than the ones implied in the estimation of Bansal and Shaliastovich (2013), which range from 76 to 113 percent.

4.2.3 Standard Deviation of Inflation and Yield News: Model-implied. So far we have shown that the model is able to match the sample inflation variance ratios. Now we ask if the model is also able to match the sample volatilities of inflation and yield shocks. To this end, Table 5 reports the 5, 50, and 95 percentiles of the posterior distribution of the standard deviation of shocks to average expected inflation over the life of the bond (SD Inflation News), standard deviation of yield shocks (SD Yield News), and the inflation variance ratios. We report these estimates for the model that includes time preference shocks. For completeness, we also report the posterior median values computed from the statistical model that does not assume (w/o SV) and does assume (w/ SV) stochastic volatility. These latter values are also reported in Table 4.

\section{[Place Table 5 about here]}

In the model and in the data, the standard deviation of inflation and yield shocks decline as maturity increases. Regarding the standard deviation of expected inflation news, note that the credible intervals contain the data moments. With respect to the standard deviation of yield news, we observe that the data moments are slightly to the left of the 5 percentile obtained from the structural model. At this point is important to recall that the inflation variance ratios are not readily available and require the use of a statistical model to compute them, which is also subject to parameter uncertainty as well as uncertainty about the latent state variables. Next we show that the macro-finance model is also able to match these two moments once we take these uncertainties into account.

Figure 5 plots the joint distribution of the annualized standard deviation of inflation and yield

news implied by the statistical model. Each point in the scatter plot is obtained by computing the standard deviation of these two shocks from a single model simulation from the posterior distribution 
of the statistical model. The solid and dash ellipsoids indicate the $90 \%$ posterior coverage for the statistical model that assumes homoskedastic (Data w/o SV) and heteroskedastic shocks (Data w/ SV), respectively. Finally, the intersection of the dashed lines denote the posterior median value of the macro-finance model that assumes time-preference shocks (Model w/ TPS).

\section{[Place Figure 5 about here]}

Many aspects of this plot are noteworthy, here we just mention two. First, the standard deviation of inflation and yield news computed from the statistical model are reliable, in the sense that the $90 \%$ posterior coverage are reasonably tight. Second, for every bond maturity, the standard deviations from the macro-finance model are well inside the ellipsoids that assume heteroskedastic shocks. These results suggest that the model is also able to jointly generate plausible values of the numerator and denominator of the inflation variance ratios.

4.2.4 Importance of the time preference shocks. It can be argued that the model will be able to generate sufficiently small inflation variance ratios by including persistent-enough shocks to the investors' time rate of preference. To address this concern, it is important to recognize that the time preference shocks not only generate news about real rates, but also affect other bond market features such as the mean, standard deviation and slope of the nominal interest rates as well as the predictability of bond returns. Appendix B.6 provides precise evidence on the trade-off between enlarging the role of the time preference shocks to match the inflation variance ratios and the model fit along other important bond yield moments. These other moments can be seen as the over-identifying restrictions that would lead us to reject the time preference shocks that allowed the model to match the inflation variance ratios.

Overall, in the data and in the model, shocks to expected inflation are not the main driver of yield innovations. We now proceed to analyze the relative contribution of each risk factor to movements in the nominal yield curve.

\subsection{Model implications for bond yield dynamics}

Even though variations in innovations to expected inflation play a limited role in driving yield shocks, the estimation of the model matches the yields well. The mean absolute pricing errors range from 
4.3 to 6.3 basis points for the different bond yields considered. These values are comparable with the pricing errors reported in the literature on macro-finance term structure models (e.g., Bikbov and Chernov 2010; Doh 2013). To highlight the importance of each state variable, in Figure 6, we show the contribution of expected consumption growth, $x_{c, t}$, expected inflation $x_{\pi, t}$, time preference shocks, $x_{\lambda, t}$, and long-run real, $\sigma_{x c, t}^{2}$, and nominal, $\sigma_{x \pi, t}^{2}$, uncertainties to the 5-Year bond yield. We do not explicitly show the contribution of the short-run real, $\sigma_{c, t}^{2}$, and nominal, $\sigma_{\pi, t}^{2}$, uncertainties because their share is too small (less than 0.5 percent) to distinguish them clearly in the graph.

\section{[Place Figure 6 about here]}

The unconditional mean of the 5-Year bond yield is 5.97 percent; however, the yield exhibits substantial variations induced by movements in the state variables. The constant $\frac{1}{n} B_{0, n}^{\$}$ sets the level at roughly 5.93 percent, and from there on the yield goes up or down depending on the relative contribution of each risk factor. In the 1970s, the decade of inflation in the United States, the 5-Year bond yield was primarily above 5.93 percent due to a rise in inflation expectations (recall that $B_{\pi}^{\$}>0$ ). In the $1980 \mathrm{~s}$, inflation expectations began to decline (see Figure 2 as a result of the program of disinflation under Chairman Volcker; however, nominal uncertainty, $\sigma_{x \pi, t}^{2}$, was at an historic high level which moved the long-term yields $\left(B_{\sigma_{x c}, n}^{\$}>0\right.$ for high $\left.n\right)$ in the opposite direction. During that period, time preference shocks were big and negative (see Figure 3), probably related to tighter financial conditions, which increased the nominal yield even more. The 1990s and 2000s were periods of relative stability in prices and, as such, $x_{\pi, t}$ and $\sigma_{x \pi, t}^{2}$ took a secondary role in explaining movements in bond yields. The 2000s were characterized by high real volatility (see Figure 2 which increased the uncertainty about future growth. To hedge this risk, the demand for risk-free assets increased, and in equilibrium, pushed the yield below 5.93 percent. $\sigma_{x c, t}^{2}$ was the dominant risk factor during the 2000s. A similar reasoning applies to the remaining bond maturities. Now we proceed to analyze the model implications for bond risk premia.

\subsection{Model implications for bond return predictability}

The model is also able to match the bond predictability results documented in Campbell and Shiller (1991) and Cochrane and Piazzesi (2005). Table6 shows that the model-implied distribution of the slope coefficients in the term spread regression of Campbell and Shiller (1991) are in line with 
the estimates based on observed data. The posterior median estimates are negative, decline with maturity, and are considerably close to the point estimates obtained from observed bond yields. Furthermore, we ran the same regressions as in Cochrane and Piazzesi (2005) and concluded that the model-simulated yields also exhibit excess bond return predictability. The posterior median coefficients were able to match the sign, pattern, and magnitude of the point estimates based on observed bond yields. The same holds for the $R^{2}$, although the posterior median estimates are somewhat larger than the ones based on observed yields.

\section{[Place Table 6 about here]}

In Section 3.3, we document that in order to match the large variations observed in the term premium dynamics, the parameters governing the standard deviation of the volatility process increased. To assess the asset pricing implications of these parameter differences, the last three columns of Table 6 replicate the same set of regressions considering only information contained in the macroeconomic variables 18 Regarding the Campbell-Shiller regressions slope, the model is unable to replicate the sign nor the increasing pattern (in absolute value) observed in the data estimates. In fact, the posterior median estimates are close to one, which is exactly the value predicted by the expectation hypothesis. However, the model is still able to generate some excess return predictability as evidenced by the $R^{2}$, though the level is considerably smaller than the one estimated with bond prices. The inclusion of asset prices in the estimation alleviates these problems by generating the extra variability needed in the stochastic volatilities of expected consumption growth and expected inflation to match the term premium dynamics.

\subsection{Model implications for expected excess returns}

In the model, movements in the one-period expected excess nominal bond returns reflect compensation for innovations to expected consumption growth and to expected inflations driven by their stochastic volatilities. Figure 7 depicts the posterior median contribution of preference risk, longrun real and inflation risk for a one-period expected excess return on a 5-Year bond 19 The total

\footnotetext{
${ }^{18}$ To this end, we use the posterior medians of the preference parameters $\Theta_{\text {pref }}$ along with the posterior distribution of $\Theta_{\text {macro }}$ obtained by only considering macro data in the estimation.

${ }^{19}$ We do not explicitly show the contribution of volatility risk since its share is too small (less than 0.55 percent) to distinguish it clearly in the graph.
} 
annualized unconditional expected excess return is positive at around 0.062 percent. However, the conditional return varies substantially in the time period considered, ranging from -0.23 percent to 0.33 percent. In the 1980s to early 1990s the major source of risk premium for holding long maturity bonds was long-run inflation risk, which accounted for approximately 71 percent of the expected excess bond returns. However, inflation-related risk premia has been practically zero since 1995. In the 1960s and 2000s the risk premium was negative and driven mostly by long-run real risk. In a stable inflation environment, interest variation comes mostly from changes in the real rates which makes long-term bonds safer assets for long-term investors. Short term bonds have higher exposure to the reinvestment risk caused by changes in the short-term real rates, and as such, they should bear a bond risk premia. As explained in the model section, bonds also command a positive risk premium driven by the preference shocks. This source of risk is constant across time and its contribution to the expected excess return is around 0.065 percent.

\section{[Place Figure 7 about here]}

\subsection{Model implications for bond term premium}

As in Bansal and Shaliastovich (2013), this model generates variability in term premia through time-varying quantity of risk driven by the long-run real and inflation volatilities. Conversely, reduced form Gaussian affine term structure models (ATSM) produce time-variation in term premia via time-varying prices of risk (e.g., Kim and Wright 2005, Bauer, Rudebusch, and Wu 2012 , Adrian et al. 2013). Central banks around the world rely on reduced form models because of their tractability and reliability. It is still an empirical open question whether time-varying quantity of risk is able to mimic the term premia dynamics based on reduced form models.

In panel (a) of Figure 8, we start by plotting the one- to five-years estimated posterior median

term premiums. As expected, the level and variation of the term premium increases with the maturity of the bond. During most of the 1960s the term premium component was practically zero; it increased throughout the 1970s, peaked in the early 1980s, and then began a declining trend for the rest of the time period. According to our estimates, the term premium became negative since the onset of the financial crisis which might be traced back to a flight to quality effect or to the large-scale asset purchases by the Federal Reserve. In this environment, negative term premium 
makes sense. We should expect a positive term premium in periods with unstable inflation and relative constant real rates such as during the 1970s and 1980s, since short-term bonds have a lower exposure to the former and a higher loading on the latter (see Figure 1). In this environment, it is safer to roll over short-term bonds that adapt faster to inflation news, than to buy long term bonds.

\section{[Place Figure 8 about here]}

In panel (b), we plot the 5-Year term premium from the model along with the estimates of Adrian et al. 2013 (ACM) and Kim and Wright 2005 (KW) for the same bond maturity. These last two models belong to ATSM class and are published by the Federal Reserve Banks of New York and St. Louis, respectively ${ }^{20}$ Panel (b) shows that the model is able to account for the level and variability of the term premium and even matches the estimates of reduced form ATSM. In fact, the correlation between the long-run risk model-implied term premium and the estimates of $\mathrm{ACM}$ and KW is about 80 and 98 percent, respectively. The correlation between ACM and KW is 69 percent. Note that the ACM estimate has been considerably higher since the last crisis, which implies a lower future path of short-term interest rates, relative to KW or our estimates.

An advantage of using a macro-finance model is that we are able trace back the movements in the term premium to changes in macroeconomic risks, while ACM and $\mathrm{KW}$ have to rely on correlations. With this in mind, panel (c) of Figure 8, depicts the contribution of long-run real, $\sigma_{x c, t}^{2}$, and nominal uncertainties, $\sigma_{x \pi, t}^{2}$, to the 5 -Year nominal term premium. Without stochastic volatilities, the term premium is constant at around 1 percent. From the 1970s to the late 1990s, long-run inflation uncertainty increased the term premium above that threshold, while real uncertainties decreased it to below 1 percent during the 1960s and even to negative values in the late 2000s. Finally, in panel $(\mathrm{d})$ we show the decomposition of the 5-Year yield into the term premium component and the expected path of short-term Treasury yields over the next five years. On average, the term premia is positive and accounts for 6 percentage of the 1-Year bond yield; it goes up to 18 percent for the 5-Year bond yield. It is instructional to note that the term premium for all bond maturities considered strongly co-moves with observed yields, which can be seen as a graphical and informal way to corroborate the failure of the expectations hypothesis presented in Table 6 .

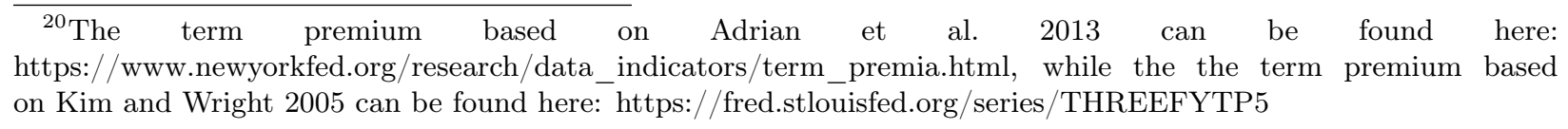


Overall, recursive preference with time-varying quantity of risk is able to generate plausible term premia dynamics without compromising the fit of the macroeconomic variables. The term premium is driven by real and nominal uncertainties and the key driver is time dependent. For the last two decades, however, nominal uncertainties have not played an important role in driving bond risk premia dynamics, while real uncertainties have played the predominant role 21

\section{Conclusion}

We develop and estimate nonlinear Bayesian state-space macro-finance endowment model that accounts for key bond market features, without resorting to an expected inflation channel that overly dominates the variation in nominal yield shocks and without sacrificing the fit of the macroeconomic aggregates. The key ingredients of the model include time preference shocks, time variation in the predictable component of consumption growth and inflation, non-neutral effects of inflation on growth, multiple stochastic volatility processes, and measurement errors and time-aggregation of consumption. With preference for early resolution of uncertainty, variations in bond risk premia are driven entirely by fluctuations in expected growth and expected inflation uncertainty. The shocks to time rate of preference, on the other hand, generate volatile and persistent fluctuations in expected short-term real rates. This in turn, allows the model to match the empirical volatility of nominal yield shocks without relying too much on the volatility of expected inflation news. Finally, we show that the time-varying volatilities in the iid component of consumption growth and inflation, the measurement error structure, and time-aggregation of consumption are negligible features for the nominal yield curve but are important for the tracking the macroeconomic series.

To assess the empirical validity of these different channels, we estimate the model using a Bayesian MCMC particle filter approach, which, among other things, addresses one crucial tradeoff inside the model: Although time preference shocks help generate news about real rates, they significantly deteriorate the model implications for bond return predictability. Importantly, the model estimation delivers smoothed estimates of the state variables, which allows us to decompose

\footnotetext{
${ }^{21}$ For parsimony, and to be conservative about the ability of macro-finance models to match term structure facts with low inflation variance ratios, our analysis has focused on the term structure market without resorting to further movements in both equity and bond market that might arise from changes in monetary policy (e.g., Campbell, Pflueger, and Viceira 2014; Song 2017) - as this is beyond the scope of this paper and, moreover, these models with monetary regimes still seem to rely too heavily on the expected inflation channel.
} 
the risk premia into their economic components in real time. We find that, for the last two decades, inflation-related risk factors have not played a predominant role in driving either expected excess bond returns or the term premium component of the nominal yield curve. The main driver seems to come from the real side of the economy.

Finally, we show that the smoothed time preference shocks series is highly correlated with the adjusted National Financial Conditions Index published by the Federal Reserve of Chicago, which measures liquidity and stresses in financial markets. Such information can be used, for instance, to help model a liquidity provider intermediary-sector to New Keynesian asset-pricing models (e.g., Kung 2015; Rudebusch and Swanson 2012) which might enlarge the real-rate channel without distorting the model implications along other dimensions. Thus, the estimation output is helpful not only for economic interpretation, but also for guiding future research by shedding light on the type of features that are worth pursuing in macro-finance models.

\section{Appendix}

\section{A Economic Model}

Appendix A is organized as follows. In Section A.1, we introduce the model set-up. In Section A.2. we present the solution to the price-consumption ratio. In Section A.3, we write the equilibrium real and nominal stochastic discount factor (SDF). In Section A.4 we solve the equilibrium nominal bond yield loadings. In sections A.5 and A.6, we derive the equilibrium expected excess bond returns and the term premium dynamics. Finally, in Section A.7, we solve the model-implied inflation variance ratios.

\section{A.1 Model set-up}

In this section, we provide a solution for the economic model. The assumed stochastic process for

the logarithm of consumption growth, $\Delta c_{t+1}=\log \frac{C_{t+1}}{C_{t}}$, and the logarithm of the inflation rate, $\pi_{t+1}$ are:

$$
\begin{aligned}
\Delta c_{t+1} & =\mu_{c}+x_{c, t}+\sigma_{c, t} \eta_{c, t+1} \\
\pi_{t+1} & =\mu_{\pi}+x_{\pi, t}+\sigma_{\pi, t} \eta_{\pi, t+1}
\end{aligned}
$$


where the process for the predictable components and the stochastic volatilities are given by:

$$
\begin{aligned}
& x_{c, t+1}=\rho_{c c} x_{c, t}+\rho_{c \pi} x_{\pi, t}+\sigma_{x c, t} \eta_{x c, t+1} \\
& x_{\pi, t+1}=\quad+\rho_{\pi \pi} x_{\pi, t}+\sigma_{x \pi, t} \eta_{x \pi, t+1}
\end{aligned}
$$

and

$$
\sigma_{i, t}=\varphi_{i} \sigma \exp \left(h_{i, t}\right), \quad \text { with } \quad h_{i, t+1}=\rho_{h_{i}} h_{i, t}+\sigma_{h_{i}} \omega_{i, t+1}
$$

All innovations are distributed according to

$$
\eta_{i, t+1}, \quad \omega_{i, t+1}, \quad \epsilon_{i, t+1} \quad \sim \text { i.i.d.N }(0,1) \quad \text { for } \quad i=\{c, \pi, x c, x \pi\}
$$

Given the assumed preference for the representative agent, the logarithm of the real stochastic discount factor $(\mathrm{SDF})$ is given by

$$
m_{t+1}=\theta \log \delta+\theta x_{\lambda, t}-\frac{\theta}{\psi} \Delta c_{t+1}+(\theta-1) r_{c, t+1}
$$

with $\theta$ defined as

$$
\theta=\frac{1-\gamma}{1-\frac{1}{\psi}}
$$

and where the time preference shocks evolve as:

$$
x_{\lambda, t+1}=\rho_{\lambda} x_{\lambda, t}+\sigma_{\lambda} \eta_{\lambda, t+1} \quad \text { with } \quad \eta_{\lambda, t+1} \sim i . i . d . N(0,1)
$$

To entertain an analytical model solution we rely on two different approximations. The first, proposed by Campbell and Shiller (1988), involves a log-linear Taylor expansion to $r_{c, t+1}$ :

$$
r_{c, t+1}=\kappa_{0}+\kappa_{1} p c_{t+1}-p c_{t}+\Delta c_{t+1}
$$

where $p c_{t+1}$ is the log price-to-consumption ratio, and $\kappa_{0}$ and $\kappa_{1}$ are constants determined endogenously by the unconditional mean of the price-consumption ratio, $\bar{p} c$, in the economy and are given by

$$
\kappa_{1}=\frac{\exp (\bar{p} c)}{1+\exp (\bar{p} c)} \quad \text { and } \quad \kappa_{0}=\log (1+\exp (\bar{p} c))-\kappa_{1} \overline{p c}
$$


The second approximation, considers a linear approximation of the volatility process around the unconditional mean of $h$ as in Schorfheide et al. (2017):

$$
\begin{aligned}
\sigma_{i, t+1}^{2} & \approx\left(\varphi_{i} \sigma\right)^{2}+2\left(\varphi_{i} \sigma\right)^{2} h_{i, t+1} \\
& =\left(\varphi_{i} \sigma\right)^{2}+2\left(\varphi_{i} \sigma\right)^{2} \rho_{h_{i}} h_{i, t}+2\left(\varphi_{i} \sigma\right)^{2} \sigma_{h_{i}} \omega_{i, t+1} \\
& \approx\left(\varphi_{i} \sigma\right)^{2}+\rho_{h_{i}}\left(\sigma_{i, t}^{2}-\left(\varphi_{i} \sigma\right)^{2}\right)+2\left(\varphi_{i} \sigma\right)^{2} \sigma_{h_{i}} \omega_{i, t+1} \\
& =\sigma_{i, 0}^{2}+\nu_{i}\left(\sigma_{i, t}^{2}-\sigma_{i, 0}^{2}\right)+\sigma_{\omega_{i}} \omega_{i, t+1} \quad \text { for } \quad i=\{c, \pi, x c, x \pi\}
\end{aligned}
$$

where we defined

$$
\nu_{i}=\rho_{h_{i}}, \quad \sigma_{i, 0}^{2}=\left(\varphi_{i} \sigma\right)^{2}, \quad \text { and } \quad \sigma_{\omega_{i}}=2\left(\varphi_{i} \sigma\right)^{2} \sigma_{h_{i}}
$$

The Euler equation is

$$
E_{t}\left[\exp \left(m_{t+1}+r_{c, t+1}\right)\right]=1
$$

which can be used to price any asset in the economy.

\section{A.2 Solution for the price-consumption ratio}

In equilibrium, the price-consumption ratio is linear in the state variables:

$$
p c_{t}=A_{0}+A_{c} x_{c, t}+A_{\pi} x_{\pi, t}+A_{\lambda} x_{\lambda, t}+A_{\sigma_{c}} \sigma_{x, t}^{2}+A_{\sigma_{\pi}} \sigma_{\pi, t}^{2}+A_{\sigma_{x c}} \sigma_{x c, t}^{2}+A_{\sigma_{x \pi}} \sigma_{x \pi, t}^{2}
$$

To solve for the coefficients $A$ s we use the log-linear approximation for $r_{c, t+1}$ in A.5 and substitute it along with the SDF in A.3 in the Euler equation in A.7). Using the method of undetermined coefficient it follows that:

$$
\begin{gathered}
A_{c}=\frac{1-\frac{1}{\psi}}{1-\kappa_{1} \rho_{c c}}, \quad A_{\pi}=\kappa_{1} \rho_{c \pi} \frac{1-\frac{1}{\psi}}{\left(1-\kappa_{1} \rho_{c c}\right)\left(1-\kappa_{1} \rho_{\pi \pi}\right)}, \quad A_{\lambda}=\frac{\rho_{\lambda}}{1-\kappa_{1} \rho_{\lambda}} \\
A_{\sigma_{c}}=\frac{(1-\gamma)\left(1-\frac{1}{\psi}\right)}{2\left(1-\kappa_{1} \nu_{c}\right)}, \quad A_{\sigma_{\pi}}=0, \quad A_{\sigma_{x c}}=\theta \frac{\left(\kappa_{1} A_{c}\right)^{2}}{2\left(1-\nu_{x c}\right)}, \quad A_{\sigma_{x \pi}}=\theta \frac{\left(\kappa_{1} A_{\pi}\right)^{2}}{2\left(1-\nu_{x \pi}\right)}
\end{gathered}
$$

and the constant is given by

$$
A_{0}=\left(1-\kappa_{1}\right)^{-1}\left\{\log \delta+\left(1-\frac{1}{\psi}\right) \mu_{c}+\kappa_{0}+\frac{1}{2} \theta\left(1+\kappa_{1} A_{\lambda}\right)^{2} \sigma_{\lambda}^{2}+\kappa_{1}\left[\sum_{i \in\left\{\sigma_{c}, \sigma_{\pi}, \sigma_{x c}, \sigma_{x \pi}\right\}}\left(1-\nu_{i}\right) A_{i} \sigma_{0, i}^{2}+\frac{1}{2} \kappa_{1} \theta A_{i}^{2} \sigma_{\omega_{i}}^{2}\right]\right\}
$$


To complete the derivation of the equilibrium price-consumption ratio we need to solve for steady state value of $p c_{t}$. To this end, we need to solve the following system of equations:

$$
\begin{aligned}
\overline{p c} & =A_{0}\left(\kappa_{1}, \kappa_{0}\right)+\sum_{i \in\left\{\sigma_{c}, \sigma_{\pi}, \sigma_{x c}, \sigma_{x \pi}\right\}} A_{i}\left(\kappa_{1}\right) \sigma_{i, 0}^{2} \\
\kappa_{1} & =\frac{\exp (\bar{p} c)}{1+\exp (\bar{p} c)} \\
\kappa_{0} & =\log (1+\exp (\bar{p} c))-\kappa_{1} \overline{p c}
\end{aligned}
$$

which can be done numerically. Having solved these constants, we now proceed to derive an expression for the SDF which we will use to price the term structure of interest rates.

\section{A.3 Expression for the real and nominal SDF}

Evaluating $r_{c, t+1}$ with the solution that we just derived for $p c_{t}$ in $\mathrm{A} .9$ and $\mathrm{A} .10$ and using the assumed process for consumption growth we can rewrite the real SDF in terms of the state variables and the underlying shocks (risk). In particular, we have:

$$
\begin{aligned}
m_{t+1}= & m_{0}+m_{c} x_{c, t}+m_{\pi} x_{\pi, t}+m_{\lambda} x_{\lambda, t}+m_{\sigma_{c}} \sigma_{x, t}^{2}+m_{\sigma_{\pi}} \sigma_{\pi, t}^{2}+m_{\sigma_{x c}} \sigma_{x c, t}^{2}+m_{\sigma_{x \pi}} \sigma_{x \pi, t}^{2} \\
& -\lambda_{c} \sigma_{c, t} \eta_{c, t+1}-\lambda_{\pi} \sigma_{\pi, t} \eta_{\pi, t+1}-\lambda_{\lambda} \sigma_{\lambda, t} \eta_{\lambda, t+1}-\lambda_{x c} \sigma_{x c, t} \eta_{x c, t+1}-\lambda_{x \pi} \sigma_{x \pi, t} \eta_{x \pi, t+1} \\
& -\sum_{i \in\left\{\sigma_{c}, \sigma_{\pi}, \sigma_{x c}, \sigma_{x \pi}\right\}} \lambda_{i} \sigma_{\omega_{i}} \omega_{i, t+1}
\end{aligned}
$$

Where the $\lambda$ 's represent the market price of each source of risk. The discount factor parameters and market price of risks are equal to:

$$
\begin{aligned}
m_{c}=-\frac{1}{\psi}, \quad m_{\pi}=0, \quad m_{\lambda}=\rho_{\lambda}, \quad m_{\sigma_{c}}=(1-\theta)\left(1-\kappa_{1} \nu_{c}\right) A_{\sigma_{c}}, \quad m_{\sigma_{\pi}}=0 \\
m_{\sigma_{x c}}=(1-\theta)\left(1-\kappa_{1} \nu_{x c}\right) A_{\sigma_{x c}}, \quad m_{\sigma_{x \pi}}=(1-\theta)\left(1-\kappa_{1} \nu_{x \pi}\right) A_{\sigma_{x \pi}}
\end{aligned}
$$

the constant is given by

$$
m_{0}=\log \delta-\frac{1}{\psi} \mu_{c}+\frac{1}{2}(1-\theta) \theta\left(1+\kappa_{1} A_{\lambda}\right)^{2} \sigma_{\lambda}^{2}+\frac{1}{2}(1-\theta) \theta \kappa_{1}^{2}\left[\sum_{i \in\left\{\sigma_{c}, \sigma_{\pi}, \sigma_{x c}, \sigma_{x \pi}\right\}} A_{i}^{2} \sigma_{\omega_{i}}^{2}\right]
$$


and the market prices of risk are

$$
\begin{gathered}
\lambda_{c}=\gamma, \quad \lambda_{\pi}=0, \quad \lambda_{\lambda}=\frac{\kappa_{1} \rho_{\lambda}-\theta}{1-\kappa_{1} \rho_{\lambda}}, \quad \lambda_{x c}=(1-\theta) \kappa_{1} A_{c}, \quad \lambda_{x \pi}=(1-\theta) \kappa_{1} A_{\pi} \\
\lambda_{\sigma_{c}}=(1-\theta) \kappa_{1} A_{\sigma_{c}}, \quad \lambda_{\sigma_{\pi}}=0, \quad \lambda_{\sigma_{x c}}=(1-\theta) \kappa_{1} A_{\sigma_{x c}}, \quad \lambda_{\sigma_{x \pi}}=(1-\theta) \kappa_{1} A_{\sigma_{x \pi}}
\end{gathered}
$$

To price nominal payoffs is useful to specify the nominal discount factor which is equal to the real one minus the inflation rate:

$$
m_{t+1}^{\$}=m_{t+1}-\pi_{t+1}
$$

We can derive a similar expression for $m_{t+1}^{\$}$ as in equation A.11, where the nominal discount factor parameters and the nominal market price of risks are equal to the real counterparts with the exception of $m_{0}^{\$}, m_{\pi}^{\$}$ and $\lambda_{\pi}^{\$}$, which are equal to:

$$
m_{0}^{\$}=m_{0}-\mu_{p}, \quad m_{\pi}^{\$}=m_{\pi}-1 \quad \text { and } \quad \lambda_{\pi}^{\$}=\lambda_{\pi}+1
$$

For convenience, it is useful to express the nominal version of equation A.11 in terms of a time $t$ expected component and innovations to the nominal SDF:

$$
\begin{aligned}
m_{t+1}^{\$}-E_{t} m_{t+1}^{\$} & =\underbrace{-\lambda_{c}^{\$} \sigma_{c, t} \eta_{c, t+1}}_{\text {short-run consumption risk }<0} \underbrace{-\lambda_{\pi}^{\$} \sigma_{\pi, t} \eta_{\pi, t+1}}_{\text {short-run inflation risk }>0} \underbrace{-\lambda_{\lambda}^{\$} \sigma_{\lambda, t} \eta_{\lambda, t+1}}_{\text {preference risk }<0} \\
& \underbrace{-\lambda_{x c}^{\$} \sigma_{x c, t} \eta_{x c, t+1}}_{\text {long-run consumption risk }<0} \underbrace{-\lambda_{x \pi}^{\$} \sigma_{x \pi, t} \eta_{x \pi, t+1}}_{\text {long-run inflation risk }>0} \\
& \underbrace{-\sum_{i \in\left\{\sigma_{c}, \sigma_{\pi}, \sigma_{x c}, \sigma_{x \pi}\right\}} \lambda_{i}^{\$} \sigma_{\omega_{i}} \omega_{i, t+1}}_{\text {Volatility risk }>0}
\end{aligned}
$$

Using the nominal discount factor in equation A.15, we can solve the equilibrium nominal yields in the economy.

\section{A.4 Solution for equilibrium nominal bond yields}

In this section we solve the yields of nominal zero-coupon bonds of different maturities. Let $P_{t, n}^{\$}$ be the time- $t$ price of an $n$-period zero-coupon nominal bond that pays one unit of numeraire in $n$ 
periods. Using the Euler equation we can write the price in logs recursively as

$$
p_{t, n}^{\$}=\log E_{t} \exp \left(m_{t+1}^{\$}+p_{t+1, n-1}^{\$}\right)
$$

where we define $p_{t, n}^{\$}=\log P_{t, n}^{\$}$. Conjecture that $p_{t, n}^{\$}$ is a linear function of the state variables

$$
p_{t, n}^{\$}=-\left(B_{0, n}^{\$}+B_{c, n}^{\$} x_{c, t}+B_{\pi, n}^{\$} x_{\pi, t}+B_{\lambda, n}^{\$} x_{\lambda, t}+B_{\sigma_{c}, n}^{\$} \sigma_{x, t}^{2}+B_{\sigma_{\pi}, n}^{\$} \sigma_{\pi, t}^{2}+B_{\sigma_{x c}, n}^{\$} \sigma_{x c, t}^{2}+B_{\sigma_{x \pi}, n}^{\$} \sigma_{x \pi, t}^{2}\right)
$$

To solve the $B$ s we again use the method of undetermined coefficients and it follows that:

$$
\begin{aligned}
B_{c, n}^{\$} & =B_{c, n-1}^{\$} \rho_{c c}-m_{c}^{\$} \\
B_{\pi, n}^{\$} & =B_{c, n-1}^{\$} \rho_{c \pi}+B_{\pi, n-1}^{\$} \rho_{\pi \pi}-m_{\pi}^{\$} \\
B_{\lambda, n}^{\$} & =B_{\lambda, n-1}^{\$} \rho_{\lambda}-m_{\lambda}^{\$} \\
B_{\sigma_{c}, n}^{\$} & =B_{\sigma_{c}, n-1}^{\$} \nu_{c}-\frac{1}{2}\left(\lambda_{c}^{\$}\right)^{2}-m_{\sigma_{c}}^{\$} \\
B_{\sigma_{\pi}, n}^{\$} & =B_{\sigma_{\pi}, n-1}^{\$} \nu_{\pi}-\frac{1}{2}\left(\lambda_{\pi}^{\$}\right)^{2}-m_{\sigma_{\pi}}^{\$} \\
B_{\sigma_{x c}, n}^{\$} & =B_{\sigma_{x c}, n-1}^{\$} \nu_{x c}-\frac{1}{2}\left(\lambda_{c}^{\$}+B_{c, n-1}^{\$}\right)^{2}-m_{\sigma_{x c}}^{\$} \\
B_{\sigma_{x \pi}, n}^{\$} & =B_{\sigma_{x \pi}, n-1}^{\$} \nu_{x \pi}-\frac{1}{2}\left(\lambda_{\pi}^{\$}+B_{\pi, n-1}^{\$}\right)^{2}-m_{\sigma_{x \pi}}^{\$} \\
B_{0, n}^{\$} & \left.=B_{0, n-1}^{\$}-\frac{1}{2}\left(\lambda_{\lambda}+B_{\lambda, n-1}^{\$}\right)^{2} \sigma_{\lambda}^{2}-\sum_{i \in\left\{\sigma_{c}, \sigma_{\pi}, \sigma_{x c}, \sigma_{x \pi}\right\}}\left[\left(B_{i, n-1}^{\$}\right) \sigma_{i, 0}^{2}\left(1-\nu_{i}\right)+\frac{1}{2}\left(\lambda_{i}+B_{i, n-1}^{\$}\right)\right)^{2} \sigma_{\omega_{i}}^{2}\right]-m_{0}^{\$}
\end{aligned}
$$

with initial conditions $B_{c, n}^{\$}=B_{\pi, n}^{\$}=B_{\lambda, n}^{\$}=B_{\sigma_{c}, n}^{\$}=B_{\sigma_{\pi}, n}^{\$}=B_{\sigma_{x c}, n}^{\$}=B_{\sigma_{x \pi}, n}^{\$}=B_{0, n}^{\$}=0$. Given the price of a zero-coupon bond, bond yields are defined $y_{t, n}^{\$}=-\frac{1}{n} \ln P_{t, n}^{\$}$. Hence,

$$
y_{t, n}^{\$}=\frac{1}{n}(B_{0, n}^{\$}+\underbrace{B_{c, n}^{\$}}_{>0} x_{c, t}+\underbrace{B_{\pi, n}^{\$}}_{>0} x_{\pi, t}+\underbrace{B_{\lambda, n}^{\$}}_{<0} x_{\lambda, t}+\underbrace{B_{\sigma_{c}, n}^{\$}}_{<0} \sigma_{c, t}^{2}+\underbrace{B_{\sigma_{\pi}, n}^{\$}}_{<0} \sigma_{\pi, t}^{2}+\underbrace{B_{\sigma_{x c}, n}^{\$}}_{<0} \sigma_{x c, t}^{2}+\underbrace{B_{\sigma_{x \pi}, n}^{\$}}_{>0 \text { for high } n} \sigma_{x \pi, t}^{2})
$$

\section{A.5 Expected excess bond returns}

Define the excess log return on buying an $n$ period bond at time $t$ and selling it at time $t+1$ as an $n-1$ period bond:

$$
r x_{r \rightarrow t+1, n}^{\$}=n y_{t, n}^{\$}-(n-1) y_{t+1, n-1}^{\$}-y_{t, 1}^{\$}
$$

As we did for the nominal SDF, the excess log return can be expressed in terms of a time $t$ expected component and return innovations:

$$
r x_{r \rightarrow t+1, n}^{\$}-E_{t} r x_{r \rightarrow t+1, n}^{\$}=-B_{c, n-1}^{\$} \sigma_{x c, t} \eta_{x c, t+1}-B_{\pi, n-1}^{\$} \sigma_{x \pi, t} \eta_{x \pi, t+1}-B_{\lambda, n-1}^{\$} \sigma_{\lambda} \eta_{\lambda, t+1}-\sum_{i \in\left\{\sigma_{c}, \sigma_{\pi}, \sigma_{x c}, \sigma_{x \pi}\right\}} B_{i, n-1}^{\$} \sigma_{\omega_{i}} \omega_{i, t+1}
$$


In the model, one-period expected excess return on nominal bonds is determined by the negative covariation between the innovations to excess log returns and the innovations to the nominal SDF in equation (A.16):

$$
\begin{aligned}
E_{t} r x_{r \rightarrow t+1, n}^{\$}+\frac{1}{2} V_{t} r x_{r \rightarrow t+1, n}^{\$}= & -\operatorname{cov}_{t}\left(m_{t+1}, r x_{r \rightarrow t+1, n}^{\$}\right) \\
& =\underbrace{-\underbrace{}_{i \in\left\{\sigma_{c}, \sigma_{\pi}, \sigma_{x c}, \sigma_{x \pi}\right\}} B_{i, n-1}^{\$} \lambda_{i} \sigma_{\omega_{i}}^{2}}_{\text {Volatility risk }} \underbrace{-B_{\lambda, n-1}^{\$} \lambda_{\lambda} \sigma_{\lambda}^{2}}_{\text {Preference risk }} \underbrace{-B_{c, n-1}^{\$} \lambda_{x c} \sigma_{x c, t}^{2}}_{\text {long-run growth risk long-run inflation risk }} \underbrace{-B_{\pi, n-1}^{\$} \lambda_{x \pi} \sigma_{x \pi, t}^{2}}
\end{aligned}
$$

\section{A.6 Term Premium}

We can decompose the $n$-period yield into expectations of average expected future short rates and average excess returns over the life of the bond:

$$
y_{t, n}^{\$}=\frac{1}{n} \sum_{i=1}^{n} E_{t} y_{t+i-1,1}^{\$}+\frac{1}{n} \sum_{i=1}^{n} E_{t} r x_{r \rightarrow t+1, n-i+1}^{\$}
$$

The last term on the right is often described as the bond's nominal term premium, and we will denote it as $t p_{t, n}^{\$}$. In this model, the term premia is time-varying due to variations in bond risk premia driven by short and long-run real and inflation volatilities. Specifically,

$$
\begin{aligned}
t p_{t, n}^{\$} & =y_{t, n}^{\$}-\frac{1}{n} \sum_{i=1}^{n} E_{t} y_{t+i-1,1}^{\$} \\
& =\frac{1}{n} B_{0, n}^{\$}-B_{0,1}^{\$}-\frac{1}{n} \sum_{i \in\{c, \pi, x c, x \pi\}} B_{\sigma_{i}, 1}^{\$}\left(n-\nu_{i} \frac{1-\nu_{i}^{n}}{1-\nu_{i}}\right) \sigma_{i, 0}^{2}+\frac{1}{n} \sum_{i \in\{x c, x \pi\}}\left(B_{\sigma_{i}, n}^{\$}-B_{\sigma_{i}, 1}^{\$} \frac{1-\nu_{i}^{n}}{1-\nu_{i}}\right) \sigma_{i, t}^{2}
\end{aligned}
$$

\section{A.7 Inflation variance ratios: Model-implied}

Equation A.21 decomposes an $n$-period yield into expectations of average expected future short rates and a term premium component. If we define the ex-ante real rate as the yield of a one-period nominal bond minus expected inflation,

$$
r_{t}=y_{t, 1}^{\$}-E_{t} \pi_{t+1}
$$

We can further decompose the bond yield as

$$
y_{t, n}^{\$}=\frac{1}{n} \sum_{i=1}^{n} E_{t} r_{t+i-1,1}+\frac{1}{n} \sum_{i=1}^{n} E_{t} \pi_{t+i}+t p_{t, n}^{\$}
$$


From this last equation it is clear that innovations in the $n$ - maturity yield from $t-1$ to $t$ are equal to the sum of news about ex-ante real rates, expected average inflation and term premium shocks:

$$
\epsilon_{y^{\$}, t}^{(n)}=\epsilon_{r, t}^{(n)}+\eta_{\pi, t}^{(n)}+\epsilon_{t p^{\$, t}}^{(n)}
$$

with

$$
\begin{array}{r}
\epsilon_{y^{\Phi}, t}^{(n)}=E_{t} y_{t, n}^{\$}-E_{t-1} y_{t, n}^{\$}, \quad \epsilon_{r, t}^{(n)}=\frac{1}{n} \sum_{i=1}^{n} E_{t} r_{t+i-1,1}-\frac{1}{n} \sum_{i=1}^{n} E_{t-1} r_{t+i-1,1} . \\
\epsilon_{t p^{\Phi, t}}^{(n)}=E_{t} t p_{t, n}^{\Phi}-E_{t-1} t p_{t, n}^{\Phi}, \quad \epsilon_{\pi, t}^{(n)}=\frac{1}{n} \sum_{i=1}^{n} E_{t} \pi_{t+i}-\frac{1}{n} \sum_{i=1}^{n} E_{t-1} \pi_{t+i} .
\end{array}
$$

Given the equilibrium solution of bond yields and the assumed process for the inflation rate we are able to derive analytical expressions for each one of this shocks. In particular, ex-ante real rates are given by

$$
\begin{aligned}
\frac{1}{n} \sum_{i=1}^{n} E_{t} r_{t+i-1,1} & =\frac{1}{n}\left(B_{0,1}^{\$}-\mu_{\pi}+\sum_{i \in\{c, \pi, x c, x \pi\}} B_{\sigma_{i}, 1}^{\$}\left(n-\nu_{i} \frac{1-\nu_{i}^{n}}{1-\nu_{i}}\right) \sigma_{i, 0}^{2}\right)+\frac{1}{n} B_{c, 1}^{\$} \frac{1-\rho_{c c}^{n}}{1-\rho_{c c}} x_{c, t}+\frac{1}{n} B_{\lambda, 1}^{\$} \frac{1-\rho_{\lambda}^{n}}{1-\rho_{\lambda}} x_{\lambda, t} \\
& +\frac{1}{n} \frac{\rho_{c \pi}}{1-\rho_{c c}} B_{c, 1}^{\$}\left[\left(1-\rho_{c c}^{n-1}\right)+\rho_{\pi \pi}\left(1-\rho_{c c}^{n-2}\right)+\rho_{\pi \pi}^{2}\left(1-\rho_{c c}^{n-3}\right)+\cdots+\rho_{\pi \pi}^{n-2}\left(1-\rho_{c c}\right)\right] x_{\pi, t} \\
& +\frac{1}{n} \sum_{i \in\{c, \pi, x c, x \pi\}} B_{\sigma_{i}, 1}^{\$} \frac{1-\nu_{i}^{n}}{1-\nu_{i}} \sigma_{i, t}^{2}
\end{aligned}
$$

Thus, we can write the innovations to this component as

$$
\begin{aligned}
\epsilon_{r, t}^{(n)} & =\frac{1}{n} B_{c, 1}^{\$} \frac{1-\rho_{c c}^{n}}{1-\rho_{c c}} \sigma_{x c, t-1} \eta_{x c, t}+\frac{1}{n} B_{\lambda, 1}^{\$} \frac{1-\rho_{\lambda}^{n}}{1-\rho_{\lambda}} \sigma_{\lambda} \eta_{\lambda, t} \\
& +\frac{1}{n} \frac{\rho_{c \pi}}{1-\rho_{c c}} B_{c, 1}^{\$}\left[\left(1-\rho_{c c}^{n-1}\right)+\rho_{\pi \pi}\left(1-\rho_{c c}^{n-2}\right)+\rho_{\pi \pi}^{2}\left(1-\rho_{c c}^{n-3}\right)+\cdots+\rho_{\pi \pi}^{n-2}\left(1-\rho_{c c}\right)\right] \sigma_{x \pi, t-1} \eta_{x \pi, t} \\
& +\frac{1}{n} \sum_{i \in\{c, \pi, x c, x \pi\}} B_{\sigma_{i}, 1}^{\$} \frac{1-\nu_{i}^{n}}{1-\nu_{i}} \sigma_{\omega_{i}} \omega_{i, t+1}
\end{aligned}
$$

Similarly, expected average inflation is equal to

$$
\frac{1}{n} \sum_{i=1}^{n} E_{t} \pi_{t+i}=\mu_{\pi}+\frac{1}{n} B_{\pi, 1}^{\$} \frac{1-\rho_{\pi \pi}^{n}}{1-\rho_{\pi \pi}} x_{\pi, t}
$$

with shocks given by

$$
\epsilon_{\pi, t}^{(n)}=\frac{1}{n} B_{\pi, 1}^{\$} \frac{1-\rho_{\pi \pi}^{n}}{1-\rho_{\pi \pi}} \sigma_{x \pi, t-1} \eta_{x \pi, t}
$$

Furthermore, equation A.22 provides an expression for the term premia. It follows that shocks to this component are

$$
\epsilon_{t p^{\Phi, t}}^{(n)}=\frac{1}{n} \sum_{i \in\{x c, x \pi\}}\left(B_{\sigma_{i}, n}^{\$}-B_{\sigma_{i}, 1}^{\$} \frac{1-\nu_{i}^{n}}{1-\nu_{i}}\right) \sigma_{\omega_{i}} \omega_{i, t+1}
$$


It is straightforward to show that the sum of $\epsilon_{r, t}^{(n)}, \epsilon_{\pi, t}^{(n)}$ and $\epsilon_{t p^{\$, t}}^{(n)}$ (given by equations A.25, A.26 and (A.27) is equal to innovations in the $n$ - maturity yield:

$$
\epsilon_{y^{\$}, t}^{(n)}=B_{c, n}^{\$} \sigma_{x c, t-1} \eta_{x c, t}+B_{\pi, n}^{\$} \sigma_{x \pi, t-1} \eta_{x \pi, t}+B_{\lambda, n}^{\$} \sigma_{\lambda} \eta_{\lambda, t}+\sum_{i \in\{c, \pi, x c, x \pi\}} B_{\sigma_{i}, n}^{\$} \sigma_{\omega_{i}} \omega_{i, t+1}
$$

Finally, the model-implied inflation variance ratios are equal to the variance of A.26 divided by the variance of A.28.

\section{B State-space representation of the macro-finance model}

In this section we describe a state-space representation of the model and its estimation procedure. In Section B.1, we derive the measurement equation, while in Section B.2, we show the state transition equation. In Section B.3, we describe the algorithm for posterior inference. Section B.4 discusses the assumed priors for the model parameters. Section B.5 and B.6 highlight the importance of the measurement error model of consumption and stochastic volatility, and the time-preference shocks, respectively.

\section{B.1 Measurement equation}

The goal of this section is to derive the coefficients of the measurement equation described in Section 2.4.1 given by:

$$
Y_{t+1}^{o}=A_{t+1}\left(D+Z s_{t+1}+Z^{v} s_{t+1}^{v}\left(h_{t+1}\right)+\Sigma^{u} u_{t+1}\right), \quad \text { with } \quad u_{t+1} \sim N(0, I)
$$

To have an expression of the observed variables in terms of model parameters and measurement errors, recall the assumed process for the macroeconomic variables and the equilibrium solutions for bond yields presented in section $\mathrm{A}$, together with the measurement-error structure described in Section 2.4.1. In particular suppose that $t+1$ is the last month of quarter $m$ and write: 


$$
\begin{aligned}
\Delta c_{t+1}^{o} & =\mu_{c}+x_{c, t}+\sigma_{c, t} \eta_{c, t+1}+\sigma_{\epsilon}\left(\epsilon_{t+1}-\epsilon_{t}\right) \\
\Delta c_{t}^{o} & =\mu_{c}+x_{c, t-1}+\sigma_{c, t-1} \eta_{c, t}+\sigma_{\epsilon}\left(\epsilon_{t}-\epsilon_{t-1}\right) \\
\Delta c_{t-1}^{o} & =\mu_{c}+x_{c, t-2}+\sigma_{c, t-2} \eta_{c, t-1}-\frac{1}{3} \sigma_{\epsilon}\left(\epsilon_{t+1}+\epsilon_{t}\right)+\frac{2}{3} \sigma_{\epsilon} \epsilon_{t-1}-\frac{2}{3} \sigma_{\epsilon} \epsilon_{t-2}+\frac{1}{3} \sigma_{\epsilon}\left(\epsilon_{t-3}+\epsilon_{t-4}\right)+\sigma_{\epsilon}^{q}\left(\epsilon_{t+1}^{q}+\epsilon_{t-2}^{q}\right) \\
\pi_{t+1}^{o} & =\mu_{\pi}+x_{\pi, t}+\sigma_{\pi, t} \eta_{\pi, t+1} \\
y_{t+1, n}^{\$, o} & =\frac{1}{n}\left(\left(B_{0, n}^{\$}+B_{c, n}^{\$} x_{c, t+1}+B_{\pi, n}^{\$} x_{\pi, t+1}+B_{\lambda, n}^{\$} x_{\lambda, t+1}+B_{\sigma_{c}, n}^{\$} \sigma_{c, t+1}^{2}+B_{\sigma_{\pi}, n}^{\$} \sigma_{\pi, t+1}^{2}+B_{\sigma_{x c}, n}^{\$} \sigma_{x c, t+1}^{2}+B_{\sigma_{x \pi}, n}^{\$} \sigma_{x \pi, t+1}^{2}\right)+\sigma_{y_{n}} \epsilon_{y_{n}, t+1}\right.
\end{aligned}
$$

where to simplify, the notation $y_{t+1, n}^{\$, o}$ denotes the one- to five-year zero-coupon bonds. Given these expressions, it is just a matter of ordering all the pieces adequately. The state vector $s_{t+1}$ and $s_{t+1}^{v}$ are given by:

$$
\begin{gathered}
s_{t+1}=\left[x_{c, t+1}, x_{c, t}, x_{c, t-1}, x_{c, t-2} \sigma_{c, t} \eta_{c, t+1}, \sigma_{c, t-1} \eta_{c, t}, \sigma_{c, t-2} \eta_{c, t-1}, \sigma_{\epsilon} \epsilon_{t+1}, \sigma_{\epsilon} \epsilon_{t}, \sigma_{\epsilon} \epsilon_{t-1}, \sigma_{\epsilon} \epsilon_{t-2}, \sigma_{\epsilon} \epsilon_{t-3}, \sigma_{\epsilon} \epsilon_{t-4}\right. \\
\left.\sigma_{\epsilon}^{q} \epsilon_{t+1}^{q}, \sigma_{\epsilon}^{q} \epsilon_{t}^{q}, \sigma_{\epsilon}^{q} \epsilon_{t-1}^{q}, \sigma_{\epsilon}^{q} \epsilon_{t-2}^{q}, x_{\pi, t+1}, x_{\pi, t}, \sigma_{\pi, t} \eta_{\pi, t+1}, x_{\lambda, t+1}, x_{\lambda, t}\right]^{\prime},
\end{gathered}
$$

and

$$
s_{t+1}^{v}=\left[\sigma_{x c, t+1}^{2}, \sigma_{x \pi, t+1}^{2}, \sigma_{c, t+1}^{2}, \sigma_{\pi, t+1}^{2}\right]^{\prime}
$$

Given these state vectors, we write the matrices $D, Z, Z^{v}$ and $\Sigma^{u}$ as follows:

$$
\begin{gathered}
Z=\left[\begin{array}{ccccccccccccccccccccccc}
0 & 1 & 0 & 0 & 1 & 0 & 0 & 1 & -1 & 0 & 0 & 0 & 0 & 0 & 0 & 0 & 0 & 0 & 0 & 0 & 0 & 0 \\
0 & 0 & 1 & 0 & 0 & 1 & 0 & 0 & 1 & -1 & 0 & 0 & 0 & 0 & 0 & 0 & 0 & 0 & 0 & 0 & 0 & 0 \\
0 & 0 & 0 & 1 & 0 & 0 & 1 & 0 & -\frac{1}{3} & -\frac{1}{3} & \frac{2}{3} & -\frac{2}{3} & \frac{1}{3} & \frac{1}{3} & 1 & 0 & 0 & -1 & 0 & 0 & 0 & 0 \\
0 & 0 & 0 & 0 & 0 & 0 & 0 & 0 & 0 & 0 & 0 & 0 & 0 & 0 & 0 & 0 & 0 & 0 & 1 & 1 & 0 & 0 \\
\frac{1}{n} B_{c, n}^{\$} & 0 & 0 & 0 & 0 & 0 & 0 & 0 & 0 & 0 & 0 & 0 & 0 & 0 & 0 & 0 & 0 & 0 & \frac{1}{n} B_{\pi, n}^{\$} & 0 & \frac{1}{n} B_{\lambda, n}^{\$} & 0
\end{array}\right] \\
D=\left[\begin{array}{c}
\mu_{c} \\
\mu_{c} \\
\mu_{c} \\
\mu_{\pi} \\
\frac{1}{n} B_{0, n}^{\$}
\end{array}\right], Z^{v}=\left[\begin{array}{cccccccc}
0 & 0 & 0 & 0 \\
0 & 0 & 0 & 0 \\
0 & 0 & 0 & 0 \\
0 & 0 & 0 & 0 \\
\frac{1}{n} B_{\sigma_{x c}, n}^{\$} & \frac{1}{n} B_{\sigma_{x \pi}, n}^{\$} & \frac{1}{n} B_{\sigma_{c}, n}^{\$} & \frac{1}{n} B_{\sigma_{\pi}, n}^{\$}
\end{array}\right], \quad \Sigma^{u}=\left[\begin{array}{cccccc}
0 & 0 & 0 & 0 & 0 \\
0 & 0 & 0 & 0 & 0 \\
0 & 0 & 0 & 0 & 0 \\
0 & 0 & 0 & 0 & 0 \\
0 & 0 & 0 & 0 & \sigma_{y_{n}}
\end{array}\right]
\end{gathered}
$$

Finally, the vector of observables $Y_{t+1}^{o}$ and the selection matrix $A_{t+1}$ can be written as:

- If $\mathrm{t}+1$ is the last month of the quarter:

$$
Y_{t+1}^{o}=\left[\begin{array}{c}
\Delta c_{t+1}^{o} \\
\Delta c_{t}^{o} \\
\Delta c_{t-1}^{o} \\
\pi_{t+1}^{o} \\
y_{t+1, n}^{\$}
\end{array}\right], \quad A_{t+1}=\left[\begin{array}{lllll}
1 & 0 & 0 & 0 & 0 \\
0 & 1 & 0 & 0 & 0 \\
0 & 0 & 1 & 0 & 0 \\
0 & 0 & 0 & 1 & 0 \\
0 & 0 & 0 & 0 & 1
\end{array}\right]^{\prime}
$$

- If $\mathrm{t}+1$ is not the last month of the quarter:

$$
Y_{t+1}^{o}=\left[\begin{array}{c}
\pi_{t+1}^{o} \\
y_{t+1, n}^{\$}
\end{array}\right], \quad A_{t+1}=\left[\begin{array}{lllll}
0 & 0 & 0 & 1 & 0 \\
0 & 0 & 0 & 0 & 1
\end{array}\right] .
$$




\section{B.2 State transition equation}

The goal of this section is to derive the coefficients of the measurement equation described in Section 2.4 .2 given by:

$$
s_{t+1}=\Phi s_{t}+v_{t+1}\left(h_{t}\right)
$$

and

$$
h_{t+1}=\Psi h_{t}+\Sigma_{h} \omega_{t+1}, \quad h_{t+1}=\left[h_{x c, t+1}, h_{x \pi, t+1}, h_{c, t+1}, h_{\pi, t+1}\right]^{\prime} \quad \text { with } \quad \omega_{t+1} \sim N(0, I) .
$$

The state variables evolve according to

$$
\begin{aligned}
& x_{c, t+1}=\rho_{c c} x_{c, t}+\rho_{c \pi} x_{\pi, t}+\sigma_{x c, t} \eta_{x c, t+1} \\
& x_{\pi, t+1}=\quad+\rho_{\pi \pi} x_{\pi, t}+\sigma_{x \pi, t} \eta_{x \pi, t+1} \\
& x_{\lambda, t+1}=\rho_{\lambda} x_{\lambda, t}+\sigma_{\lambda} \eta_{\lambda, t+1}
\end{aligned}
$$

Hence, $\Phi$ and $v_{t+1}\left(h_{t}\right)$ just make sure that these dynamics are preserved with zeros and ones in the adequate places:

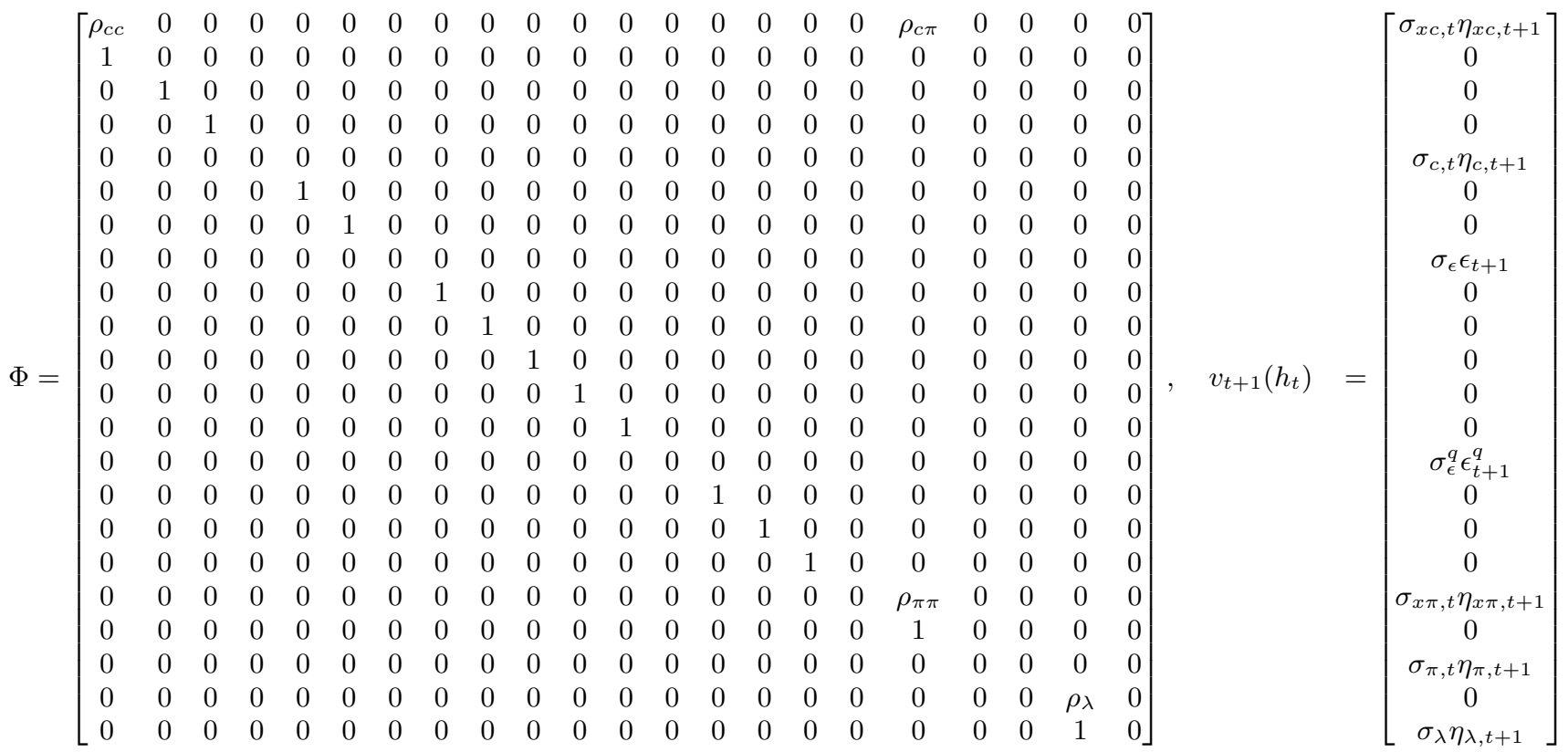

Finally, $\Psi, \Sigma_{h}$ and $\omega_{t+1}$ can be written as 


$$
\Psi=\left[\begin{array}{cccc}
\rho_{h_{x c}} & 0 & 0 & 0 \\
0 & \rho_{h_{x \pi}} & 0 & 0 \\
0 & 0 & \rho_{h_{c}} & 0 \\
0 & 0 & 0 & \rho_{h_{\pi}}
\end{array}\right], \quad \Psi=\left[\begin{array}{cccc}
\sigma_{h_{x c}} & 0 & 0 & 0 \\
0 & \sigma_{h_{x \pi}} & 0 & 0 \\
0 & 0 & \sigma_{h_{c}} & 0 \\
0 & 0 & 0 & \sigma_{h_{\pi}}
\end{array}\right], \quad \omega_{t+1}=\left[\begin{array}{c}
\omega_{x c, t+1} \\
\omega_{x \pi, t+1} \\
\omega_{c, t+1} \\
\omega_{\pi, t+1}
\end{array}\right]
$$

with

$$
\sigma_{i, t}=\varphi_{i} \sigma \exp \left(h_{i, t}\right), \quad \text { for } \quad i=\{c, \pi, x c, x \pi\}
$$

and we normalized $\varphi_{c}=1$.

\section{B.3 Posterior inference}

For posterior inference we use a Bayesian MCMC particle filter to the state-space representation described by equations B.1, B.2 and B.3, which is linear conditional on the log volatilities $h_{t}$. We briefly summarize the main steps below; for further details, refer to Algorithm 13 in Herbst and Schorfheide (2015).

1. We initialize the Markov Chain at some parameter values $\Theta^{0}$.

2. Given $\Theta^{k}$

(a) Initialization. At time $t=0$, we draw $M$ particles $\left\{h_{0}^{j}\right\}_{j=1}^{M}$ from the unconditional distribution of equation B.3. Conditional on each particle $h_{0}^{j}$, we generate $s_{0}^{j}$ from the unconditional distribution of equation B.2. We set each particle weight to $\pi_{0}^{j}=\frac{1}{M}$, $j=1, \ldots, M$.

(b) Recursion. For $t=1, \ldots, T$ :

i. Forecasting $s_{t}$. We propagate each particle $h_{t-1}^{j}$ using the law of motion in equation B.3 to get $h_{t}^{j}$. Given $s_{t-1}^{j}$ and $\left(h_{t-1}^{j}, h_{t}^{j}\right)$ we run one iteration of the Kalman filter using the state-space system given by equations B.1 and B.2, which is conditionally linear. This step delivers the distribution of $s_{t}^{j}$, denoted by $p\left(s_{t} \mid y_{t}^{o}, s_{t-1}^{j}, h_{t-1}^{j}, h_{t}^{j}\right)$, which is normal with mean $s_{t \mid t}^{j}$ and variance $P_{t \mid t}^{j}$. Hence,

$$
s_{t}^{j} \sim N\left(s_{t \mid t}^{j}, P_{t \mid t}^{j}\right) .
$$

ii. Forecasting $y_{t}$. We compute the incremental weights $\tilde{\omega}_{t}^{j}$ according to

$$
\tilde{\pi}_{t}^{j}=\pi_{t-1}^{j} \times p\left(y_{t}^{o} \mid \tilde{y}_{t \mid t-1}^{j}, F_{t \mid t-1}^{j}\right)
$$

where the Kalman filter step in $i$ delivers $\tilde{y}_{t \mid t-1}^{j}$ and $F_{t \mid t-1}^{j}$. The likelihood $p\left(y_{t}^{o} \mid \tilde{y}_{t \mid t-1}^{j}, F_{t \mid t-1}^{j}\right)$ is conditionally Gaussian and follows from the measurement equation B.1.

iii. Updating. We define the normalized weights by

$$
\pi_{t}^{j}=\frac{\tilde{\pi}_{t}^{j}}{\sum_{i=1}^{M} \tilde{\pi}_{t}^{i}}
$$


and resample the particles $h_{t}^{j}$ and states $s_{t}^{j}$ using multinomial resampling with weights $\pi_{t}^{j}$.

(c) Likelihood Approximation. The approximation of the log-likelihood function is given by

$$
\ln \hat{p}\left(y_{t}^{o} \mid Y_{1: t-1}^{o}\right)=\ln \hat{p}\left(y_{t-1}^{o} \mid Y_{1: t-2}^{o}\right)+\ln \left(\sum_{i=1}^{M} \tilde{\pi}_{t}^{j}\right)
$$

(d) Metropolis-Hastings algorithm Once we are able to approximate the likelihood function, we use a standard random walk Metropolis-Hastings algorithm to obtain a new parameter draw $\Theta^{k+1}$. See Algorithm 18 in Herbst and Schorfheide (2015) for further details regarding this step.

(e) We repeat steps (a) to (b) $N_{\text {sim }}$ times.

In the implementation, we used 10,000 particles $(M=10,000)$, generated 50,000 draws $\left(N_{\text {sim }}=50,000\right)$ and set the burn in period at 25,000 . We targeted an acceptance rate of approximately 30 percent. In addition, we checked that the results do not changed if we increase the number of particles.

\section{B.4 Prior distribution}

In general, we attempted to restrict the parameter space to economically plausible values while at the same time be as uninformative as possible. The first columns of Table 1 show the assumed prior distribution, as well as the 5 and 95 percentiles. We will first discuss the priors of the macroeconomic parameters, $\Theta_{\text {macro }}$, and then continue with the priors of the preference parameters $\Theta_{\text {pref }}$.

The priors for the persistence parameters of the predictable components of consumption growth, $\rho_{c c}$, and inflation, $\rho_{\pi, \pi}$, as well as the prior for the parameter that measures the nonneutral effect of inflation on growth, $\rho_{c \pi}$, are distributed uniformly over the interval $(-1,1)$. This range includes magnitudes that imply a zero effect of expected inflation on expected consumption growth, as well as a near iid or unit root process for the macroeconomic variables. The parameter $\varphi_{\pi}$ sets the standard deviation of the iid component of inflation relative to consumption growth. Here we use a prior that is uniform on the interval $(0,3)$ and encompasses values that allows $\pi_{t}$ to be more or less volatile than $\Delta c_{t}$. A similar logic applies for $\varphi_{x c}$ and $\varphi_{x \pi}$ with respect to the conditional means of consumption, $x_{c, t}$, and inflation, $x_{\pi, t}$, respectively. The priors for average annualized consumption growth, $\mu_{c}$, and inflation, $\mu_{\pi}$, are normally distributed with a 90 percent interval ranging from \pm 10 percent. 
Regarding the preferences parameters, $\Theta_{\text {pref }}$, our priors are quite agnostic in that they cover values commonly reported in other studies and try not to affect the asset pricing implications of the model. For instance, we do not restrict the preference of the representative household to have early, $\theta<0$, or late, $\theta>0$, resolution of uncertainty: the prior for the intertemporal elasticity of substitution, $\psi$, and the risk aversion coefficient, $\gamma$, cover values below and above one. The prior

for the discount factor, $\delta$, covers steady state annualized values for the one period risk free rate between 0.5 and 5 percent. Furthermore, the priors for $\rho_{\lambda}$ and $\varphi_{\lambda}$ have the same support as the persistence and standard deviation parameters of the macroeconomic dynamics, where we defined $\sigma_{\lambda}=\varphi_{\lambda} \sigma_{c}$ in (2). Finally, we fixed the standard deviation of the yields measurement error, $\sigma_{y^{n}}$, at 10 percent of the yields' sample standard deviation, a value consistent with the work of Bekaert, Hodrick, and Marshall (1997).

\section{B.5 Role of measurement error model of consumption and stochastic volatility}

In Section 3.3.2 of the main text, we documented that the posterior median estimates of $\left\{\rho_{c c}, \rho_{c \pi}, \rho_{\pi \pi}\right\}$ are remarkably similar with or without including bond prices in the estimation. The result is mostly driven by the assumed measurement error model for consumption. To see this, in Table B1, we show the 5, 50, 95 percentiles of the posterior distribution of the persistent parameters under three different specifications of the consumption growth and the inflation process. In (a) we assume homoskedastic innovations and that consumption growth is measured without any errors, (i.e., $c_{t}^{o}=c_{t}$ ). In (b) we add the measurement error model of consumption, while in (c), we assume the measurement error model of consumption and consider short- and long-run stochastic volatility. In the last specification, we impose a common stochastic volatility process (i.e., $h_{c, t}=h_{x c, t}$ and $h_{\pi, t}=h_{x \pi, t}$ ). Furthermore, in the last column of the table, we report the log marginal data densities to provide formal support in terms of model fit for each specification. In all cases we only consider macro data in the estimation.

\section{[Place Table B1 about here]}

Without the measurement error model, the posterior median $\rho_{c c}$ is equal to -0.20 in order to match the monthly negative autocorrelation observed in the data of -0.17 . Nevertheless, this version of the model cannot reconcile the monthly negative autocorrelation with a positive autocorrelation 
observed at a quarterly or annual frequency. Accounting for measurement errors in consumption that average out every quarter alleviates this problem. Under this specification, the posterior median of $\rho_{c c}$ jumps to 0.90 , and the fit of the model measured by the log marginal data density improves significantly from 5855 to 5870 . At the same time, the persistence parameter associated with expected inflation, $\rho_{\pi \pi}$, remains largely unchanged at a value of 0.91 , while the parameter that measures the effect of expected inflation on expected growth, $\rho_{c \pi}$, increases from -0.13 to -0.02. Adding stochastic volatility to the process of the macro variables improves the model fit considerably, the posterior uncertainty decreases (tighter credible intervals) and the persistence parameters $\rho_{c c}$ and $\rho_{\pi \pi}$ jump to 0.94 and 0.98 , respectively ${ }^{22} \rho_{c \pi}$ remained at -0.02 . In terms of model fit, adding stochastic volatility increases the log marginal data density from 5870 to $6055{ }^{23}$ Finally, it is important to highlight that under the three different specification, $\rho_{c \pi}$, is negative and significant as suggested by the 90 percent credible interval.

\section{B.6 The importance of the time preference shocks in the nominal yield curve.}

In this subsection we provide specific evidence on the tradeoff between enlarging the role of the time preference shocks to match the inflation variance ratios and the model fit along other important bond yield moments. These other moments serve as the overidentifying restrictions that would lead us to reject the time preference shocks that allowed the model to succeed.

In this paper, the time preference shocks evolve according to:

$$
x_{\lambda, t+1}=\rho_{\lambda} x_{\lambda, t}+\sigma_{\lambda} \eta_{\lambda, t+1} \quad \text { with } \quad \eta_{\lambda, t+1} \sim i . i . d . N(0,1)
$$

The exercise consist of gradually increasing the persistence parameter, $\rho_{\lambda}$, and the standard deviation of the shocks, $\sigma_{\lambda}$, from relatively small to large values in order to analyze their effect on the dynamics of the nominal term structure. To this end, we fixed the remaining parameters at their posterior median values.

\footnotetext{
${ }^{22}$ The intuition for why the $\rho_{c c}$ and $\rho_{\pi \pi}$ increases goes as follows. The presence of stochastic volatility allows for sharp fluctuations in $\Delta c_{t+1}^{o}$ and $\pi_{t+1}^{o}$ by incurring in similar movements in their conditional variance without the need of large temporary shocks by increasing $\sigma$ and $\varphi_{\pi}$ and reducing the estimates of the persistent parameters.

${ }^{23}$ In specification (Me. Sv.) We impose a common stochastic volatility process (i.e. $h_{c, t}=h_{x c, t}$ and $h_{\pi, t}=h_{x \pi, t}$ ). If we allow for four different stochastic volatility processes, the confidence interval for most of the parameters increased considerably and the log marginal data density decreases to 5761 . With these evidence, we conclude that there is not enough information in consumption growth and inflation to identify four different stochastic volatilities
} 
B.6.1 The inflation variance ratios are decreasing in $\rho_{\lambda}$ and $\sigma_{\lambda}$. Panel A) of Table B2 shows that by making the shocks big enough and their persistence large enough, the model is able to match the model inflation variance ratios. For example, if $\rho_{\lambda}$ increases from 0.5 to 0.999 the inflation variance ratio for the 5 -Year bond decreases from 73 to 11 percent. However, enlarging these shocks have first order effects on other important bond yield moments. We will briefly describe how enlarging the time preference shocks channel distorts the slope of the term structure and decreases the model-implied predictability of bond returns.

\section{[Place Table B2 about here]}

B.6.2 The slope of the nominal term structure is increasing in $\rho_{\lambda}$ and $\sigma_{\lambda}$. Panel B) of Table B2 shows that enlarging the time preference shocks channel generates an increasingly steep term structure. Section 2.3 .2 of the paper provides the theoretical reason for this result. The slope is defined as the difference between the mean of the five-year and one-year bond yield. For example, if we increase $\rho_{\lambda}$ from 0.5 to 0.999 the slope increases from 16 to 418 basis points, which is clearly at odds with the value observed in the data of 67 basis points.

B.6.3 Bond risk premia is decreasing in $\rho_{\lambda}$ and $\sigma_{\lambda}$. Panel C) of Table B2 shows that enlarging the time preference shocks channel mutes the model-implied predictability of bond returns. This mechanism is more subtle and we address this issue next. Campbell and Shiller (1991) test the expectation hypothesis by running the following regression

$$
y_{t+12, n-12}-y_{t, n}=\alpha_{n}+\beta_{n} \frac{12}{n-12}\left(y_{t, n}-y_{t, 12}\right)+\epsilon_{t+12} \quad \text { for } \quad n \in\{24,36,48,60\}
$$

Under the expectation hypothesis $\beta_{n}=1$ which relates the slope of the term structure to expected changes in the bond yield, $y_{t, n}$, from time period $t$ to $t+12$. The second column of panel C) shows that in the data $\beta_{n}$ is not only different from 1 , but it is also negative. This result implies that a positively sloped term structure predicts a decrease in future bond yields. It follows that on average the bond term premium has to be positively related to the slope of the nominal yield curve. To see 
this note that that the following equation holds

$$
E_{t}\left[y_{t+12, n-12}-y_{t, n}\right]+t p_{t, n}=\frac{12}{n-12}\left(y_{t, n}-y_{t, 12}\right) .
$$

Since time preference shocks affect the slope of the yield curve, $y_{t, n}-y_{t, 12}$, but do not affect the bond term premium, $t p_{t, n},{ }^{24}$ it has to be the case that an increase in the slope via this channel (say by increasing $\rho_{\lambda}$ and $\sigma_{\lambda}$ to match the inflation variance ratios) will also increase the expected change in the bond yield, $E_{t}\left[y_{t+12, n-12}-y_{t, n}\right]$, which in turn will move the estimated slope, $\beta_{n}$, towards one. Panel C) of Table B2, shows these movements in the slope of the Campbell- Shiller regression. For example, if we increase $\rho_{\lambda}$ from 0.5 to 0.999 , the slope $\beta_{n}$ for the five-year bond increases from -1.68 to 0.08 . Hence, the model could fail to simultaneously match the inflation variance ratios proposed by Duffee (2016) and quantitatively account for the failure of the expectation hypothesis as identified in Campbell and Shiller (1991).

In sum, these two exercise show that there is a clear tradeoff in matching the inflation variance ratios and other bond market features.

\section{Inflation variance ratios: Data}

In this section we provide further details on how to compute the inflation variance ratios from the data. In Section C.1, we show how to compute news to expected average inflation and to nominal bond yields, which are the main ingredients for the inflation variance ratios derived from the data. In Section C.2, we write the state-space representation of the model, while in Section C.3, we present the estimation procedure and the prior and posterior distribution of the model parameters. Finally, in Section C.4, we carry out some robustnesses checks based on a quarterly version of the model.

\section{C.1 News to expected average inflation and to nominal bond yields}

From equation 12 in Section 2.3.2, we showed that innovations to the $n$ - maturity yield from $t-1$ to $t$ are equal to the sum of news about ex-ante real rates, expected average inflation and term

\footnotetext{
${ }^{24}$ In the model, variations in bond term premium are driven entirely by time-varying conditional volatilities of the predictable components of consumption growth and inflation and are not affected by the time preference shock channel.
} 
premium shocks:

$$
\epsilon_{y^{\S}, t}^{(n)}=\epsilon_{r, t}^{(n)}+\epsilon_{\pi, t}^{(n)}+\epsilon_{t p^{\Phi, t}}^{(n)}
$$

Next we provide further details on how to obtained an estimate of $\epsilon_{\pi, t}^{(n)}$. For consistency, we extracted one- to five-year inflation forecasts from the same model assumed in the macro-finance model:

$$
\begin{gathered}
\pi_{t+1}=\mu_{\pi}+x_{t}+\sigma_{\pi, t} \eta_{\pi, t} \\
x_{t}=\rho x_{t-1}+\sigma_{x, t} \eta_{x, t}
\end{gathered}
$$

with

$$
\eta_{\pi, t}, \quad \eta_{x, t} \sim i . i . d . N(0,1)
$$

Given this process for inflation, time $t$ inflation expectations for $j$ months ahead is given by

$$
E_{t} \pi_{t+j}=\mu_{\pi}+\rho^{j-1} x_{t}
$$

Hence the expected inflation innovation from $t$ to $t+1$ in period $t+j$ can be written as

$$
E_{t+1}-E_{t} \pi_{t+j}=\rho^{j-1} \sigma_{x, t} \eta_{x, t+1}
$$

From here, we can express the innovations to expected average inflation as

$$
\epsilon_{\pi, t}^{(n)}=E_{t+1}-E_{t} \frac{1}{n} \sum_{i=1}^{m} \pi_{t+j}=\frac{1}{n} \frac{1-\rho^{n}}{1-\rho} \sigma_{x, t} \eta_{x, t, t+1}
$$

Alternatively, to compute shocks to the $n$-period yield, $\epsilon_{y^{\$}, t}^{(n)}$, we assume that bond yields follow martingales

$$
y_{t}^{(n)}=y_{t-1}^{(n)}+\sigma_{y^{(n)}, t} \eta_{y^{(n)}, t+1} \quad \text { with } \quad \eta_{y^{(n)}, t+1} \sim i . i . d . N(0,1)
$$

where

$$
\epsilon_{y^{\S}, t}^{(n)}=\sigma_{y^{(n)}, t} \eta_{y^{(n), t}, t+1}
$$


Finally, the process for the stochastic volatilities is also the same as in the baseline model

$$
\sigma_{i, t}=\sigma_{i} \exp \left(h_{i, t}\right), \quad \text { with } \quad h_{i, t+1}=\rho_{h_{i}} h_{i, t}+\sigma_{h_{i}} \omega_{i, t+1} \text { and } \omega_{i, t+1} \sim i . i . d . N(0,1) \text { for } i=\{\pi, x, y\}
$$

assuming a common volatility structure for the bond yields.

\section{C.2 State-space representation}

The state-space representation is given by the measurement equation:

$$
Y_{t+1}^{o}=A_{t+1}\left(D+Z \alpha_{t+1}+H \epsilon_{t+1}\right) \quad \text { with } \quad \epsilon_{t+1} \sim N(0, I)
$$

and the state transition equation is

$$
\alpha_{t+1}=T \alpha_{t}+\eta_{t+1}\left(h_{t}\right) \quad \text { and } \quad h_{t+1}=\Psi h_{t}+\Sigma_{h} \omega_{t+1} \quad \text { with } \quad \omega_{t+1} \sim N(0, I)
$$

with $h_{t+1}=\left[h_{\pi, t+1}, h_{x, t+1}, h_{y, t+1}\right]^{\prime}$.

Following Duffee (2016), we include information from the surveys of market practitioners that are released in a different (quarterly) frequency to sharpen inference. In particular, we consider the CPI forecasts from the Survey of Professional Forecasters of one to four quarters ahead. To this end, we assume that the forecasts are published at the second month of the quarter, which is consistent with the release date of the Federal Reserve Bank of Philadelphia. To gain some insight into how to include this information, let $t$ be the second month of the quarter. Then the market practitioners make an inflation forecast for say, one quarter ahead, starting from period $t+1$, which we label as $\tilde{\pi}_{t+1}^{o, 1}$. Using the process for inflation in equation C.2 the following relation follows:

$$
\tilde{\pi}_{t+1}^{o, 1}=E_{t}\left[\pi_{t+2}+\pi_{t+3}+\pi_{t+4}\right]=3 \mu_{p}+\tilde{\rho}_{1} x_{t}
$$

where to simplify the notation we defined $\tilde{\rho}_{1}=\rho+\rho^{2}+\rho^{3}$. The same applies for the inflation forecasts

of two $\tilde{\pi}_{t+1}^{o, 2}$, three $\tilde{\pi}_{t+1}^{o, 3}$ and four $\tilde{\pi}_{t+1}^{o, 4}$ quarters ahead. Following this notation, the observed variables 
in terms of the state variables are given by

$$
\begin{gathered}
\pi_{t+1}^{o}=\mu+x_{t}+\sigma_{\pi, t} \eta_{\pi, t+1} \\
\tilde{\pi}_{t+1}^{o, 1}=3 \mu+\tilde{\rho}_{1} x_{t}+\sigma_{\epsilon}^{1} \epsilon_{t+1}^{1} \\
\tilde{\pi}_{t+1}^{o, 2}=3 \mu+\tilde{\rho}_{2} x_{t}+\sigma_{\epsilon}^{2} \epsilon_{t+1}^{2} \\
\tilde{\pi}_{t+1}^{o, 3}=3 \mu+\tilde{\rho}_{3} x_{t}+\sigma_{\epsilon}^{3} \epsilon_{t+1}^{3} \\
\tilde{\pi}_{t+1}^{o, 4}=3 \mu+\tilde{\rho}_{4} x_{t}+\sigma_{\epsilon}^{4} \epsilon_{t+1}^{4} \\
y_{t+1, n}^{\$, o}=y_{t+1, n}^{\$}+\sigma_{\epsilon, y_{n}} \epsilon_{y_{n}, t+1}
\end{gathered}
$$

where to simplify the notation $y_{t+1, n}^{\$, o}$ denotes the one- to five-year zero-coupon bonds and we assumed that the survey consensus forecasts are measured with errors. The standard deviation of the measurement errors are free parameters given by $\sigma_{\epsilon}^{i}$. To be consistent with the macro-finance model, we set the standard deviation of the yield's measurement error, $\sigma_{\epsilon, y_{n}}$, at 10 percent of their sample standard deviation, similar to the assumed value for the macro-finance model. Furthermore, we defined

$$
\tilde{\rho}_{2}=\rho^{4}+\rho^{5}+\rho^{6}, \quad \tilde{\rho}_{3}=\rho^{7}+\rho^{8}+\rho^{9}, \quad \text { and } \quad \tilde{\rho}_{4}=\rho^{10}+\rho^{11}+\rho^{12} .
$$

The state vector can be written as

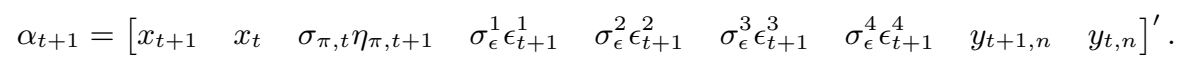

Hence, the $D, Z$ and $H$ can be written as

$$
D=\left[\begin{array}{c}
\mu \\
3 \mu \\
3 \mu \\
3 \mu \\
3 \mu \\
0
\end{array}\right], \quad Z=\left[\begin{array}{ccccccccc}
0 & 1 & 1 & 0 & 0 & 0 & 0 & 0 & 0 \\
0 & \tilde{\rho}_{1} & 0 & 1 & 0 & 0 & 0 & 0 & 0 \\
0 & \tilde{\rho}_{2} & 0 & 0 & 1 & 0 & 0 & 0 & 0 \\
0 & \tilde{\rho}_{3} & 0 & 0 & 0 & 1 & 0 & 0 & 0 \\
0 & \tilde{\rho}_{4} & 0 & 0 & 0 & 0 & 1 & 0 & 0 \\
0 & 0 & 0 & 0 & 0 & 0 & 0 & 1 & 0
\end{array}\right], \quad H=\left[\begin{array}{cccccc}
0 & 0 & 0 & 0 & 0 & 0 \\
0 & 0 & 0 & 0 & 0 & 0 \\
0 & 0 & 0 & 0 & 0 & 0 \\
0 & 0 & 0 & 0 & 0 & 0 \\
0 & 0 & 0 & 0 & 0 & 0 \\
0 & 0 & 0 & 0 & 0 & \sigma_{\epsilon, y_{n}}
\end{array}\right]
$$

Finally, the vector of observables $Y_{t+1}^{o}$ and the selection matrix $A_{t+1}$ can be written as:

- If $\mathrm{t}+1$ is the last month of the quarter:

$$
Y_{t+1}^{o}=\left[\begin{array}{c}
\pi_{t+1}^{o} \\
\tilde{\pi}_{t+1}^{o, 1} \\
\tilde{\pi}_{t+2}^{o, 2} \\
\tilde{\pi}_{t+1}^{o, 3} \\
\tilde{\pi}_{t+1}^{o, 4} \\
y_{t+1, n}^{\$, o}
\end{array}\right], \quad A_{t+1}=\left[\begin{array}{cccccc}
1 & 0 & 0 & 0 & 0 & 0 \\
0 & 1 & 0 & 0 & 0 & 0 \\
0 & 0 & 1 & 0 & 0 & 0 \\
0 & 0 & 0 & 1 & 0 & 0 \\
0 & 0 & 0 & 0 & 1 & 0 \\
0 & 0 & 0 & 0 & 0 & 1
\end{array}\right]^{\prime}
$$


- If $\mathrm{t}+1$ is not the last month of the quarter:

$$
Y_{t+1}^{o}=\left[\begin{array}{c}
\pi_{t+1}^{o} \\
y_{t+1, n}^{\$}
\end{array}\right], \quad A_{t+1}=\left[\begin{array}{llllll}
1 & 0 & 0 & 0 & 0 & 0 \\
0 & 0 & 0 & 0 & 0 & 1
\end{array}\right] .
$$

Regarding the coefficients associated with the state transition equation, we write $T$ and $\eta_{t+1}\left(h_{t}\right)$ as follows

$$
T=\left[\begin{array}{ccccccccc}
\rho & 0 & 0 & 0 & 0 & 0 & 0 & 0 & 0 \\
1 & 0 & 0 & 0 & 0 & 0 & 0 & 0 & 0 \\
0 & 0 & 0 & 0 & 0 & 0 & 0 & 0 & 0 \\
0 & 0 & 0 & 0 & 0 & 0 & 0 & 0 & 0 \\
0 & 0 & 0 & 0 & 0 & 0 & 0 & 0 & 0 \\
0 & 0 & 0 & 0 & 0 & 0 & 0 & 0 & 0 \\
0 & 0 & 0 & 0 & 0 & 0 & 0 & 0 & 0 \\
0 & 0 & 0 & 0 & 0 & 0 & 0 & 1 & 0 \\
0 & 0 & 0 & 0 & 0 & 0 & 0 & 1 & 0
\end{array}\right], \quad \eta_{t+1}\left(h_{t}\right)=\left[\begin{array}{c}
\sigma_{x, t} \eta_{x, t} \\
0 \\
\sigma_{\pi, t} \eta_{\pi, t+1} \\
\sigma_{\epsilon}^{1} \epsilon_{t+1}^{1} \\
\sigma_{\epsilon}^{2} \epsilon_{t+1}^{2} \\
\sigma_{\epsilon}^{3} \epsilon_{t+1}^{3} \\
\sigma_{\epsilon}^{4} \epsilon_{t+1}^{4} \\
\sigma_{y^{(n)}, t} \eta_{y(n), t+1}^{4} \\
0
\end{array}\right]
$$

Finally, the matrices associated with the log volatility process are:

$$
\Psi=\left[\begin{array}{ccc}
\rho_{h_{\pi}} & 0 & 0 \\
0 & \rho_{h_{x}} & 0 \\
0 & 0 & \rho_{h_{y}}
\end{array}\right], \quad \Psi=\left[\begin{array}{ccc}
\sigma_{h_{\pi}} & 0 & 0 \\
0 & \sigma_{h_{x}} & 0 \\
0 & 0 & \sigma_{h_{y}}
\end{array}\right], \quad \omega_{t+1}=\left[\begin{array}{c}
\omega_{\pi, t+1} \\
\omega_{x, t+1} \\
\omega_{y, t+1}
\end{array}\right]
$$

with

$$
\sigma_{i, t}=\sigma_{i} \exp \left(h_{i, t}\right), \quad \text { for } \quad i=\left\{\pi, x, y^{(n)}\right\}
$$

\section{C.3 Posterior inference}

We use a Metropolis-within-Gibbs sampler for posterior inference. To this end, it is useful to sample from the conditional distributions for a subset of parameters and latent variables conditional on all the remaining ones. In particular, we iterate over three conditional distributions. We summarize the parameters and latent volatilities accordingly:

$$
\begin{gathered}
\Theta_{\pi}=\left\{\mu, \sigma_{\pi}, \rho, \sigma_{x}, \sigma_{y^{(12)}}, \sigma_{y^{(24)}}, \sigma_{y^{(36)}}, \sigma_{y^{(48)}}, \sigma_{y^{(60)}}, \sigma_{\epsilon}^{1}, \sigma_{\epsilon}^{2}, \sigma_{\epsilon}^{3}, \sigma_{\epsilon}^{4},\right\}, \\
\Theta_{h}=\left\{\rho_{h_{\pi}}, \sigma_{h_{\pi}}, \rho_{h_{x}}, \sigma_{h_{x}}, \rho_{h_{y}}, \sigma_{h_{y}}\right\}, \quad H^{1: T}=\left\{h_{\pi}^{1: T}, h_{x}^{1: T}, h_{y}^{1: T}\right\}
\end{gathered}
$$

We use Markov chain Monte Carlo (MCMC) algorithm that iterates over the three conditional distributions to generate a sequence of draws

$$
\left\{\Theta_{\pi}^{(s)},\left(H^{1: T}\right)^{(s)}, \Theta_{h}^{(s)}\right\}_{s=1}^{N_{s i m}}
$$


from the posterior distribution defined by Bayes' Theorem as:

$$
p\left(\Theta_{\pi}, \Theta_{h}, H^{1: T} \mid Y^{o}\right) \propto p\left(Y^{o} \mid \Theta_{\pi}, \Theta_{h}, H^{1: T}\right) p\left(H^{1: T} \mid \Theta_{\pi}, \Theta_{h}\right) p\left(\Theta_{\pi}, \Theta_{h}\right)
$$

The main steps of the Metropolis-within-Gibbs sampler are as follows:

1. We initialize the sample starting from $\left\{\left(H^{1: T}\right)^{(0)}, \Theta_{h}^{(0)}\right\}$.

2. We use a random walk Metropolis-Hastings step to draw from the posterior of $\Theta_{\pi}$ conditional on $\left\{Y^{o},\left(H^{1: T}\right)^{(s-1)}, \Theta_{h}^{(s-1)}\right\}$. For the covariance matrix of the proposal distribution, we follow Schorfheide (2005) and use the negative inverse of the posterior density Hessian evaluated at the mode. We target an acceptance rate of approximately 30 percent by adjusting the scaling factor of the proposal distribution accordingly.

3. We sample the stochastic volatilities using the procedure of Kim, Shephard, and Chib (1998) conditioning on $\left\{Y^{o}, \Theta_{\pi}^{(s)}, \Theta_{h}^{(s-1)}\right\}$.

4. We draw $\Theta_{h}$ using a standard Gibbs sampling approach conditional on $\left\{\left(H^{1: T}\right)^{(s)}\right\}$. We retain draws that imply roots inside the unit circle.

We generated 50,000 draws and we set the burn in period at 25,000 draws. Table C1 shows the prior and posterior distribution.

\section{[Place Table C1 about here]}

\section{C.4 Robustness}

As a robustness check, we repeated the same exercise for a quarterly version of the homoskedastic and heteroskedastic model considering three different measures of inflation expectations reported by SPF. Table $\mathrm{C} 2$ shows the estimated inflation variance ratios 25 In panel (a) we replicate the same exercise as in Table 4 , but using quarterly frequency instead of using mix-frequency data. In panel (b), we use the GDP deflator (PGDP) as the measure of the inflation rate and we use the survey consensus forecasts of current-quarter inflation (nowcasts) rather than actual observed inflation as our measure of $\pi^{o}$. In panel (c), we repeated the same exercise as in panel (b) with CPI inflation instead of PGDP. Overall, expected inflation news does not contribute much to the variation of bond innovations (below 30 percent) for all bond maturities considered. Allowing for

\footnotetext{
${ }^{25}$ Parameter estimates are available upon request.
} 
stochastic volatility almost doubles the inflation variance ratios relative to the homoskedastic case, and increases the model fit; furthermore, all inflation variance ratios are below 50 percent.

[Place Table C2 about here] 


\section{References}

Adrian, T., R. K. Crump, and E. Moench. 2013. Pricing the term structure with linear regressions. Journal of Financial Economics 110:110-138.

Albuquerque, R., M. Eichenbaum, V. Luo, and S. Rebelo. 2016. Valuation Risk and Asset Pricing. The Journal of Finance 71:2861-2904.

Andrieu, C., A. Doucet, and R. Holenstein. 2010. Particle markov chain monte carlo methods. Journal of the Royal Statistical Society: Series B (Statistical Methodology) 72:269-342.

Ang, A., G. Bekaert, and M. Wei. 2007. Do macro variables, asset markets, or surveys forecast inflation better? Journal of monetary Economics 54:1163-1212.

Bansal, R., and I. Shaliastovich. 2013. A long-run risks explanation of predictability puzzles in bond and currency markets. Review of Financial Studies 26:1-33.

Bansal, R., and A. Yaron. 2004. Risks for the long run: A potential resolution of asset pricing puzzles. The Journal of Finance 59:1481-1509.

Bauer, M. D., G. D. Rudebusch, and J. C. Wu. 2012. Correcting estimation bias in dynamic term structure models. Journal of Business 86 Economic Statistics 30:454-467.

Bekaert, G., R. J. Hodrick, and D. A. Marshall. 1997. On biases in tests of the expectations hypothesis of the term structure of interest rates. Journal of Financial Economics 44:309-348.

Bikbov, R., and M. Chernov. 2010. No-arbitrage macroeconomic determinants of the yield curve. Journal of Econometrics 159:166-182.

Campbell, J. Y., and J. Ammer. 1993. What moves the stock and bond markets? A variance decomposition for long-term asset returns. The Journal of Finance 48:3-37.

Campbell, J. Y., C. Pflueger, and L. M. Viceira. 2014. Monetary policy drivers of bond and equity risks. Tech. rep., National Bureau of Economic Research.

Campbell, J. Y., and R. J. Shiller. 1988. The dividend-price ratio and expectations of future dividends and discount factors. Review of financial studies 1:195-228. 
Campbell, J. Y., and R. J. Shiller. 1991. Yield spreads and interest rate movements: A bird's eye view. The Review of Economic Studies 58:495-514.

Cieslak, A. 2016. Short-rate expectations and unexpected returns in Treasury bonds. unpublished paper, Duke University .

Cochrane, J. H., and M. Piazzesi. 2005. Bond risk premia. The American economic review 95:138160.

Creal, D. D., and J. C. Wu. 2016. Bond risk premia in consumption-based models. Tech. rep., National Bureau of Economic Research.

Doh, T. 2013. Long-Run Risks In The Term Structure Of Interest Rates: Estimation. Journal of Applied Econometrics 28:478-497.

Drost, F. C., and T. E. Nijman. 1993. Temporal aggregation of GARCH processes. Econometrica: Journal of the Econometric Society pp. 909-927.

Duffee, G. R. 2002. Term premia and interest rate forecasts in affine models. The Journal of Finance $57: 405-443$.

Duffee, G. R. 2016. Expected inflation and other determinants of Treasury yields. Johns Hopkins University, Department of Economics Working Paper .

Epstein, L. G., and S. E. Zin. 1989. Substitution, risk aversion, and the temporal behavior of consumption and asset returns: A theoretical framework. Econometrica: Journal of the Econometric Society pp. 937-969.

Eraker, B., I. Shaliastovich, and W. Wang. 2016. Durable goods, inflation risk, and equilibrium asset prices. Review of Financial Studies 29:193-231.

Fernández-Villaverde, J., and J. F. Rubio-Ramírez. 2007. Estimating macroeconomic models: A likelihood approach. The Review of Economic Studies 74:1059-1087.

Herbst, E. P., and F. Schorfheide. 2015. Bayesian Estimation of DSGE Models. Princeton University Press. 
Kim, D. H., and J. H. Wright. 2005. An arbitrage-free three-factor term structure model and the recent behavior of long-term yields and distant-horizon forward rates .

Kim, S., N. Shephard, and S. Chib. 1998. Stochastic volatility: likelihood inference and comparison with ARCH models. The Review of Economic Studies 65:361-393.

Kung, H. 2015. Macroeconomic linkages between monetary policy and the term structure of interest rates. Journal of Financial Economics 115:42-57.

Piazzesi, M., and M. Schneider. 2007. Equilibrium yield curves. In NBER Macroeconomics Annual 2006, Volume 21, pp. 389-472. MIT Press.

Rudebusch, G. D., and E. T. Swanson. 2012. The bond premium in a dsge model with long-run real and nominal. American Economic Journal: Macroeconomics 4:105-143.

Schorfheide, F. 2005. Learning and monetary policy shifts. Review of Economic dynamics 8:392-419.

Schorfheide, F., D. Song, and A. Yaron. 2017. Identifying long-run risks: A bayesian mixedfrequency approach. forthcoming, Econometrica .

Song, D. 2017. Bond market exposures to macroeconomic and monetary policy risks. The Review of Financial Studies 30:2761-2817.

Stock, J. H., and M. W. Watson. 2007. Why has US inflation become harder to forecast? Journal of Money, Credit and banking 39:3-33.

Verdelhan, A. 2010. A habit-based explanation of the exchange rate risk premium. The Journal of Finance 65:123-146.

Wachter, J. A. 2006. A consumption-based model of the term structure of interest rates. Journal of Financial Economics 79:365-399.

Wilcox, D. W. 1992. The construction of US consumption data: some facts and their implications for empirical work. The American economic review pp. 922-941. 


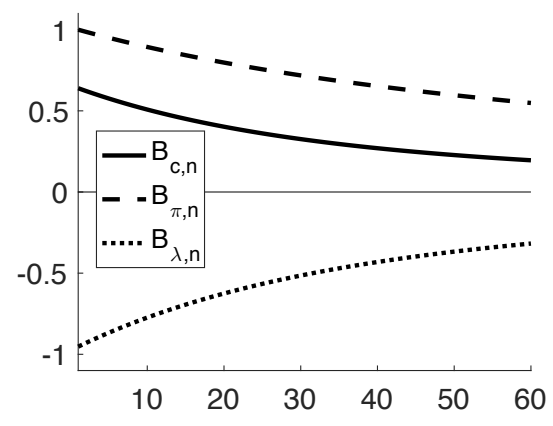

(a) $B_{c, n}^{\$}, B_{\pi, n}^{\$}$, and $B_{\lambda, n}^{\$}$

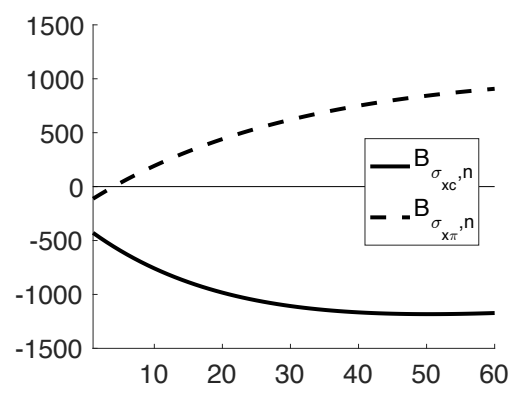

(b) $B_{\sigma_{x c}, n}^{\$}$ and $B_{\sigma_{x \pi}, n}^{\$}$

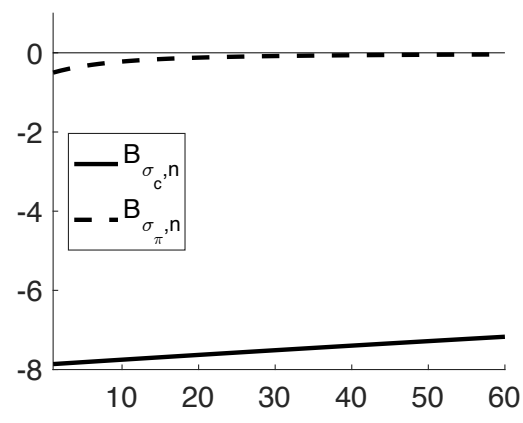

(c) $B_{\sigma_{c}, n}^{\$}$ and $B_{\sigma_{\pi}, n}^{\$}$

Figure 1.

Equilibrium nominal bond yield loadings. This figure shows model implied nominal bond yield loadings evaluated at posterior median values reported in Table1. Panel (a) shows the loadings with respect to expected consumption growth, $x_{c, t}$, expected inflation, $x_{\pi, t}$ and time preference shocks, $x_{\lambda, t}$, respectively. Panel (b) shows the loadings with respect to long-run real, $\sigma_{x c, t}^{2}$, and nominal, $\sigma_{x \pi, t}^{2}$, uncertainties. Panel (c) shows the loadings with respect to short-run real, $\sigma_{c, t}^{2}$ and nominal, $\sigma_{\pi, t}^{2}$, uncertainties. Maturity is in months. 


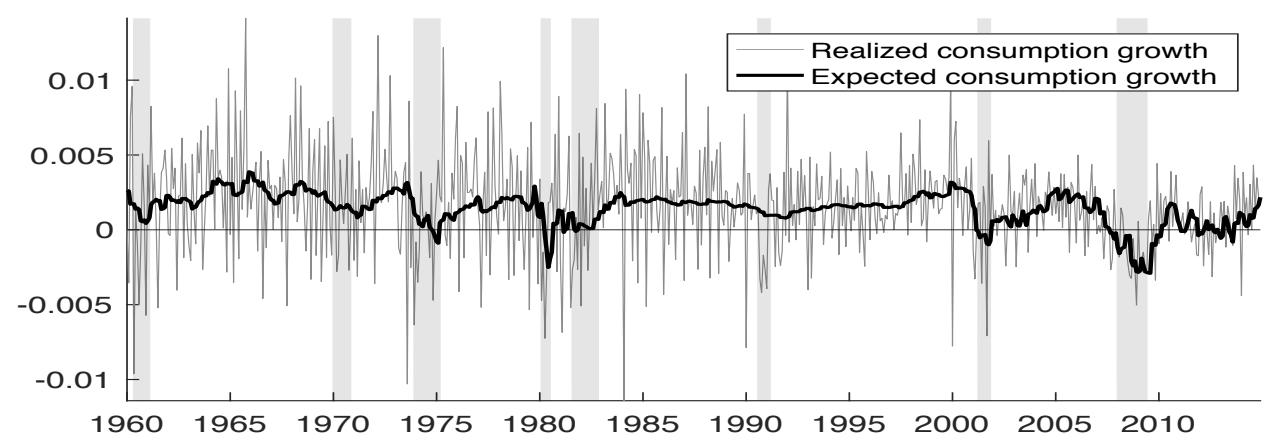

(a) Long-Run Growth $x_{c}$

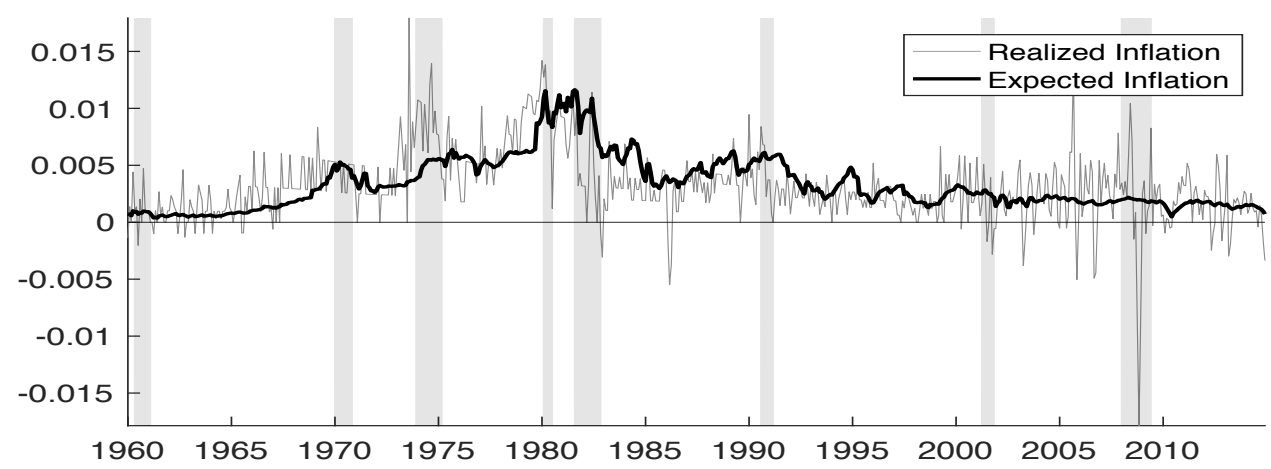

(b) Long-Run Inflation $x_{\pi}$

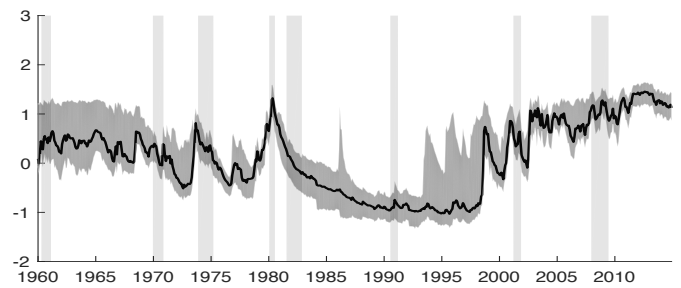

(c) Long-Run Growth Vol $h_{x c}$

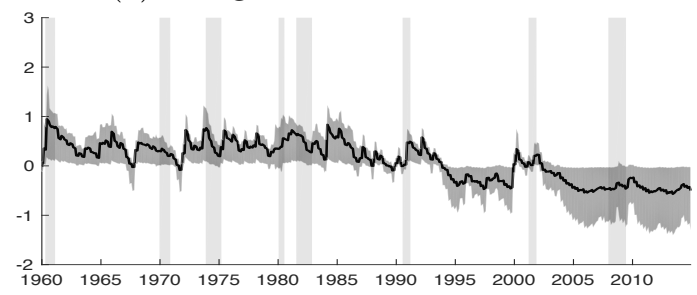

(e) Short-Run Growth Vol $h_{c}$

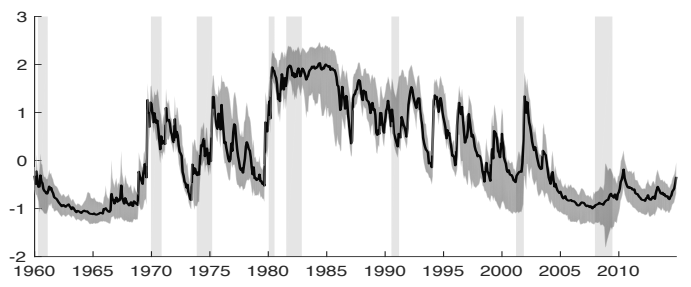

(d) Long-Run Inflation Vol $h_{x \pi}$

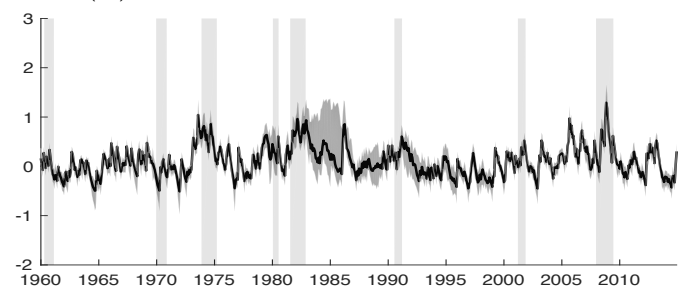

(f) Short-Run Inflation $\mathrm{Vol} h_{\pi}$

\section{Figure 2.}

Macroeconomic latent state variables. This figure shows the smoothed mean and volatility states filtered from the macro-finance model. In panels (a) and (b), we overlay include the observed series of consumption growth and inflation, respectively. In panel (c), (d), (e), and (f) we plot the filtered log volatilities. Dark gray shaded areas correspond to the 90 percent credible intervals. Light shaded bars represent recessions as defined by the National Bureau of Economic Research. The estimation sample is from 1960:M1 to 2014M12. 


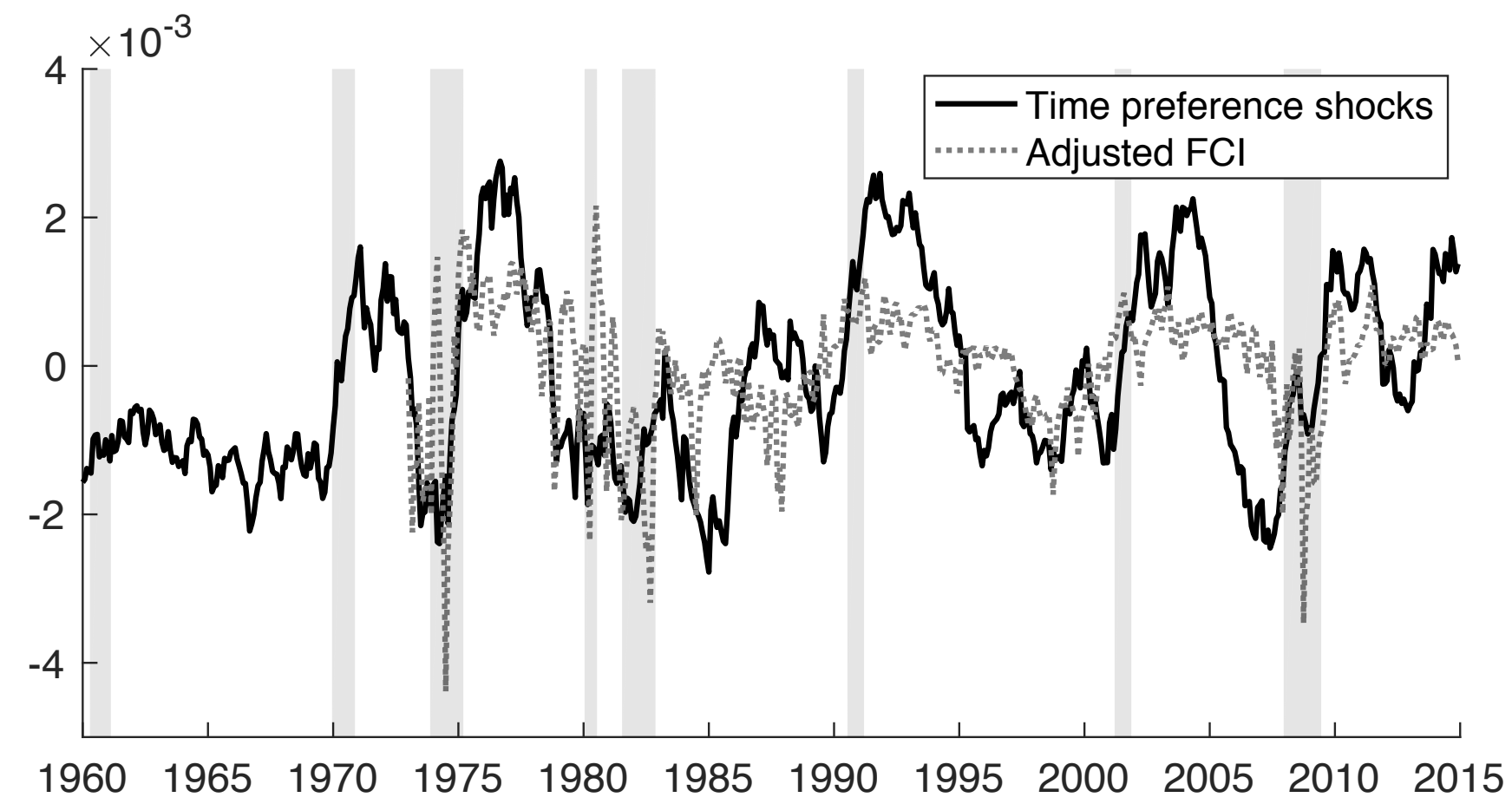

Figure 3.

Time preference shocks and financial condition Index. This figure shows the latent time preference shocks state variable filtered from the macro-finance model along with the adjusted National Financial Conditions Index published by the Federal Reserve Bank of Chicago. Light shaded bars represent recessions as defined by the National Bureau of Economic Research. The estimation sample is from 1960:M1 to 2014M12. The adjusted National Financial Conditions Index starts in 1973 and is published at https://alfred.stlouisfed.org/series?seid=ANFCI. 


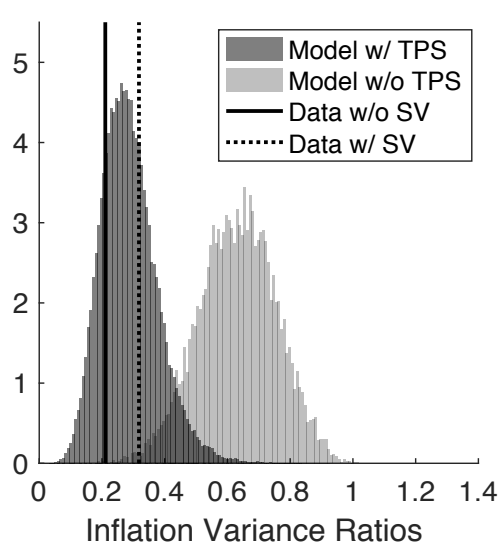

(a) $1 \mathrm{y}$

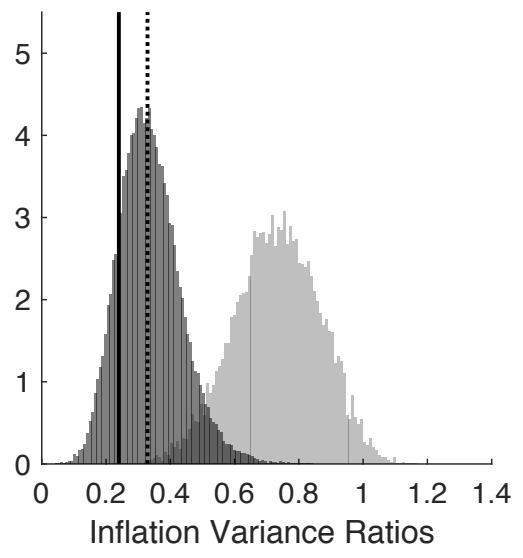

(b) $2 y$

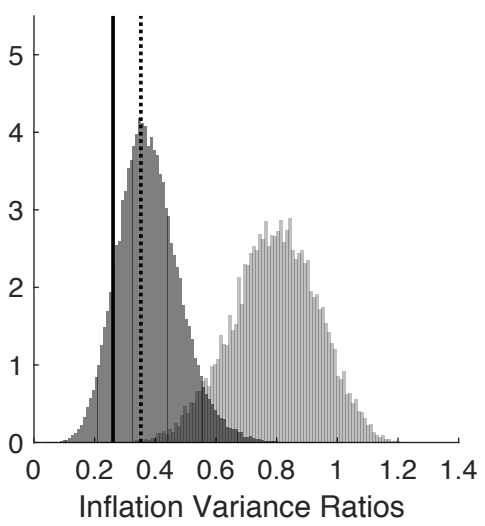

(c) $3 y$

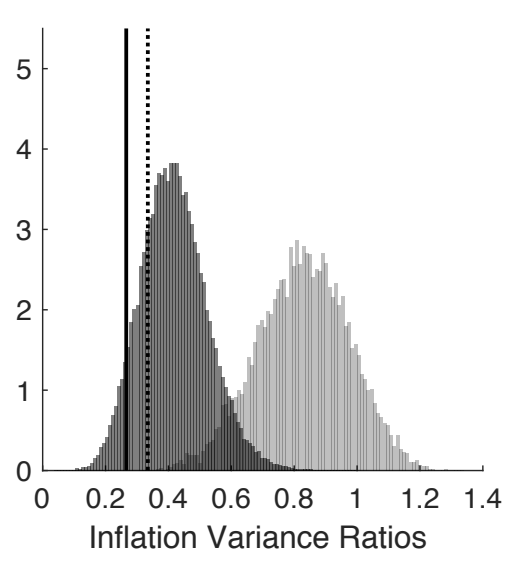

(d) $4 y$

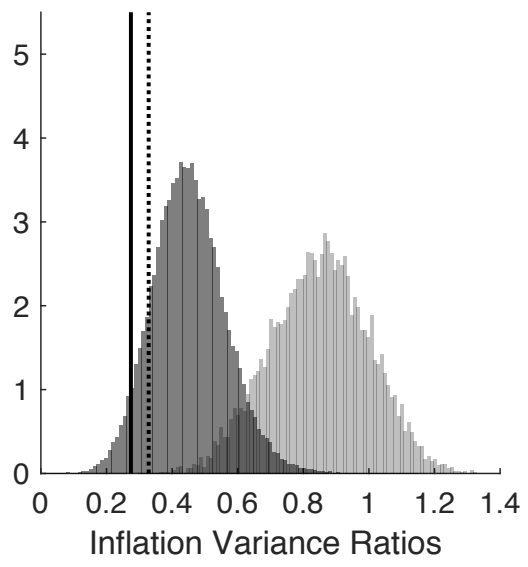

(e) $5 \mathrm{y}$

Figure 4.

Inflation variance ratios: Macro-finance model. This figure shows the probability density function of the inflation variance ratios obtained from two different versions of the macro-finance model and for different bond maturities. The dark shaded density is based on the macro-finance model that includes time preference shocks (Model w/ TPS), while the light shaded density does not include them (Model w/o TPS). The straight and dashed black lines denote the posterior median inflation variance ratios from the statistical model that assumes homoskedastic (Data w/o SV) and heteroskedastic shocks (Data w/ SV), respectively. To compute these estimates, we draw the parameters from their posterior distribution and simulate 10,000 data sets of 660 (555) periods for the macro-finance model (statistical models). 


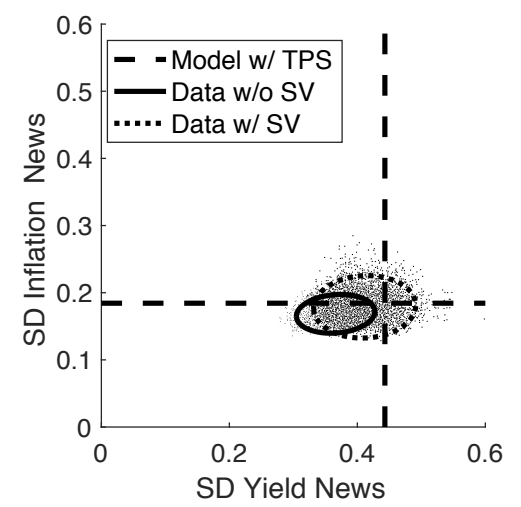

(a) $1 \mathrm{y}$

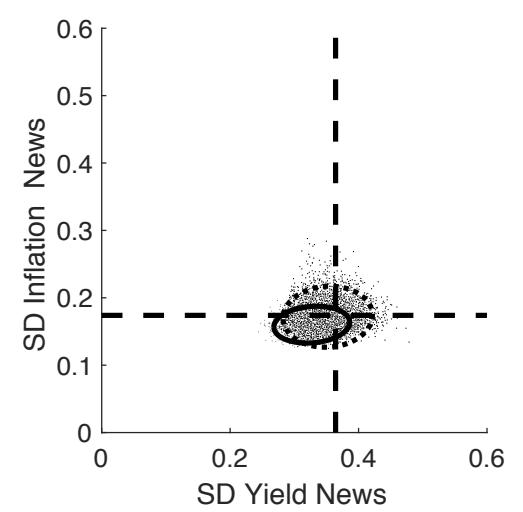

(b) $2 y$

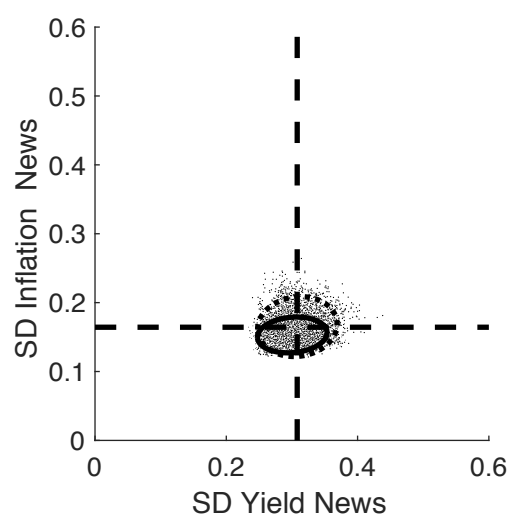

(c) $3 y$

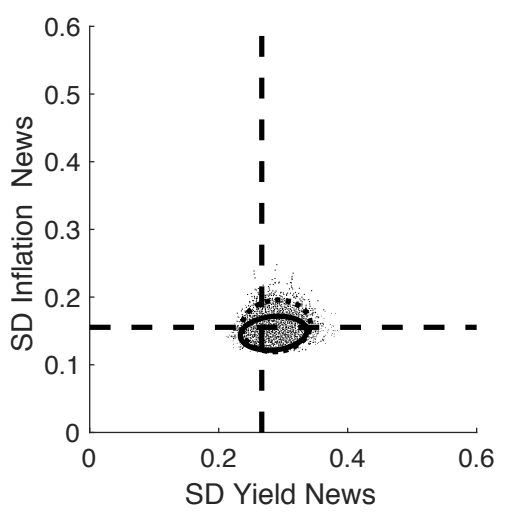

(d) $4 \mathrm{y}$

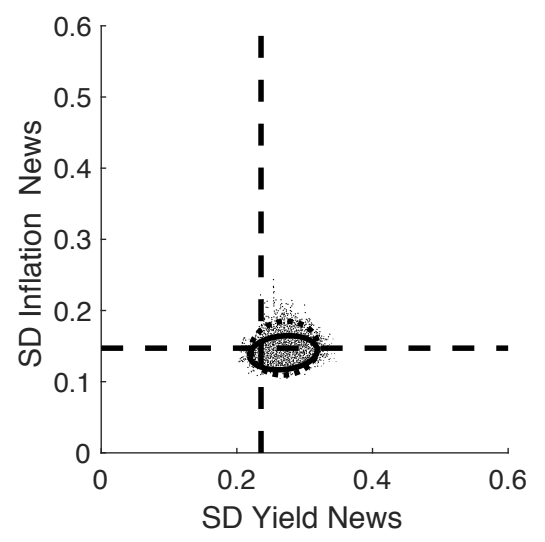

(e) $5 y$

Figure 5.

Standard deviation of yield and inflation shocks. This figure shows the model-implied joint distribution of the standard deviation of shocks to average expected inflation (SD Inflation News) and the standard deviation of yield shocks (SD Yield News) for the statistical model. Each dot is obtained by computing the standard deviation of these two shocks from a single model simulation. The solid and dash lines indicate $90 \%$ posterior coverage ellipsoids for the statistical model that assumes homoskedastic (Data w/o SV) and heteroskedastic shocks (Data w/ SV), respectively. The dashed lines denote the posterior median value of the macro-finance model that assumes timepreference shocks (Model w/ TPS). Standard deviations are in annualized percent/month. To compute these estimates, we draw the parameters from their posterior distribution and simulate 10,000 data sets of 660 (555) periods each for the macro-finance models (statistical models). 


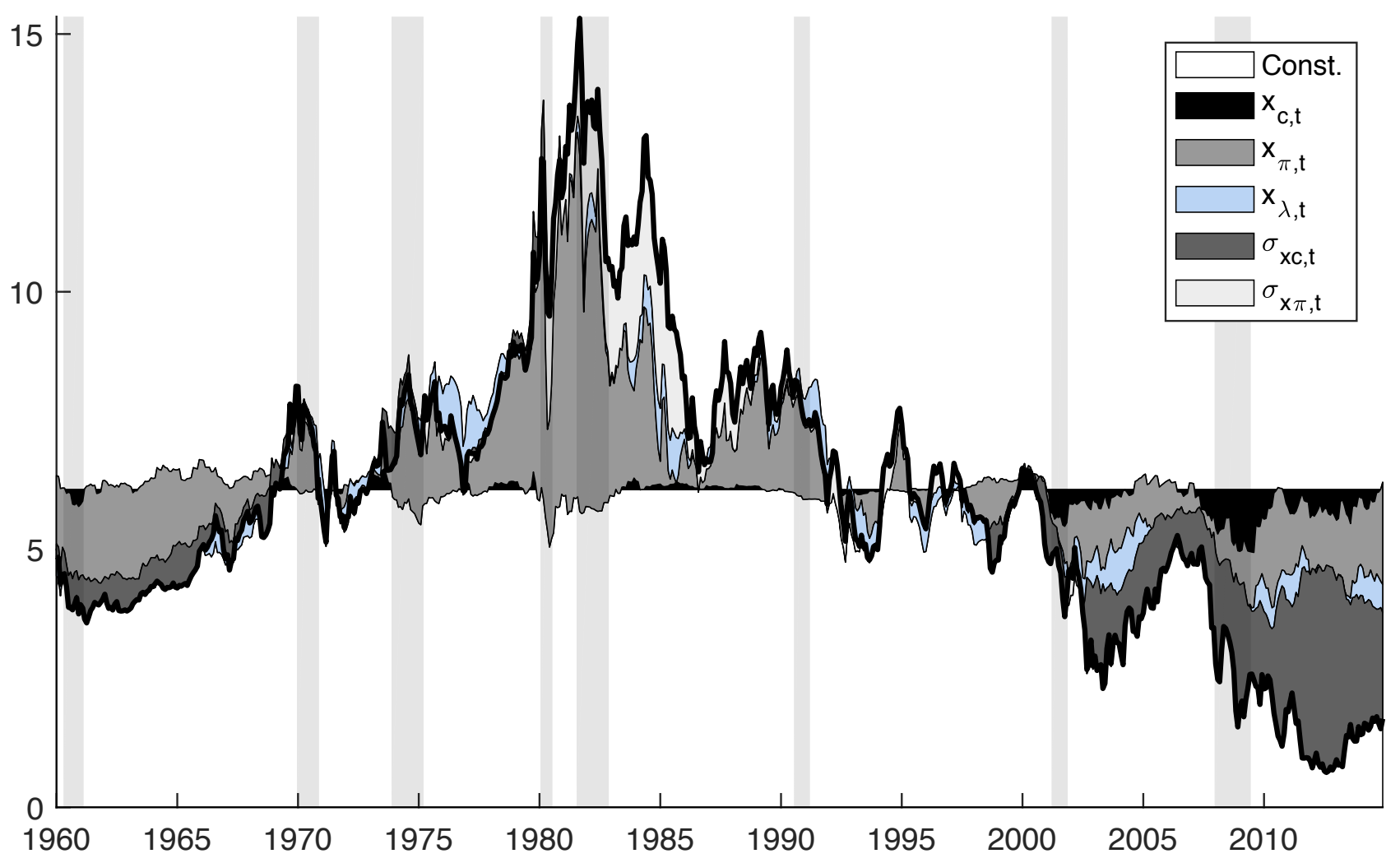

Figure 6.

Decomposition of the 5-Year bond. This figure shows the model-implied nominal bond yields. We show the posterior median contribution of each state variable to the 5-Year bond yield. Light shaded bars represent recessions as defined by the National Bureau of Economic Research. The annualized mean absolute pricing errors $E\left(\left|u_{y, t}\right| \mid Y^{o}\right)$ in basis points are equal to 5.66 (for the 1Year bond, 1y), 5.97 (2y), $5.71(3 y), 4.26$ (4y) and 6.26 (5y). The estimation sample is from 1960:M1 to $2014 \mathrm{M} 12$. 


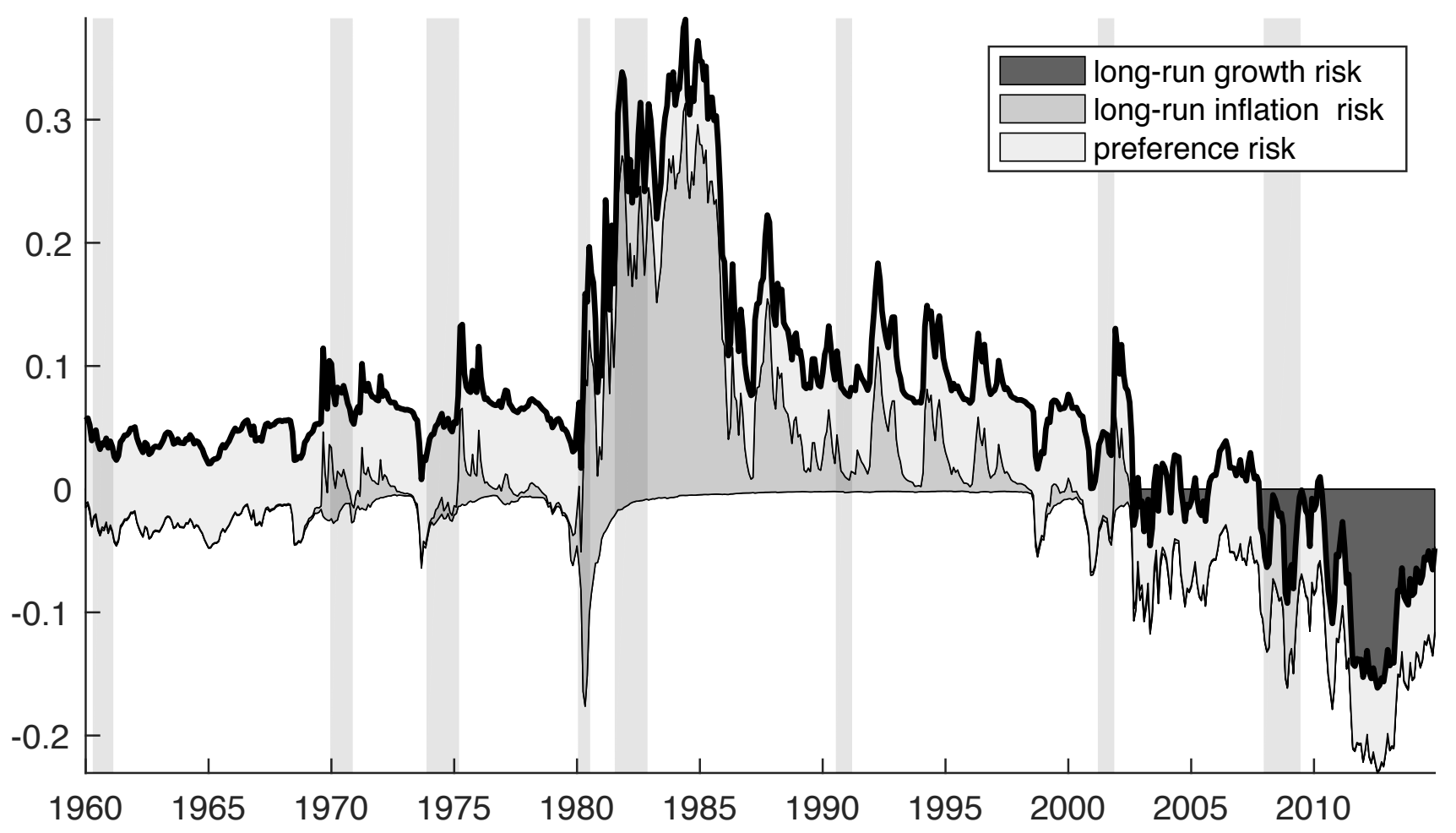

Figure 7.

Decomposition of one period expected excess bond returns for the 5-Year bond. This figure shows the contribution of long-run growth risk, long-run inflation risk, and preference risk to the one-period expected excess bond returns for the 5-Year bond. Light shaded bars represent recessions as defined by the National Bureau of Economic Research. The estimation sample is from 1960:M1 to 2014M12. 


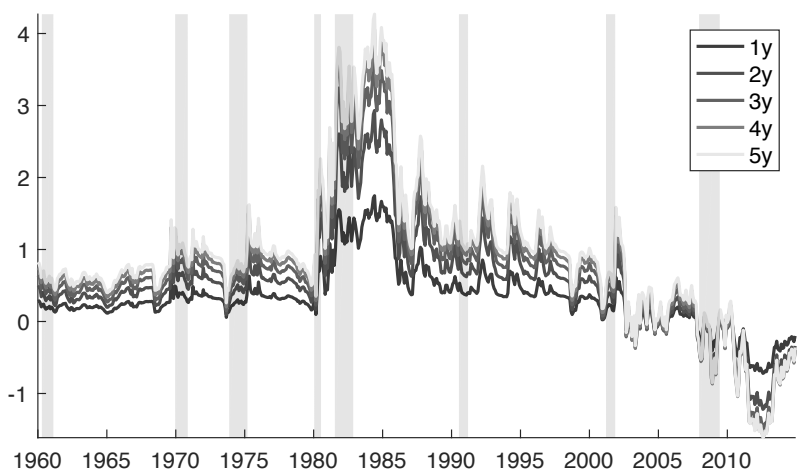

(a) Term premium by bond maturity

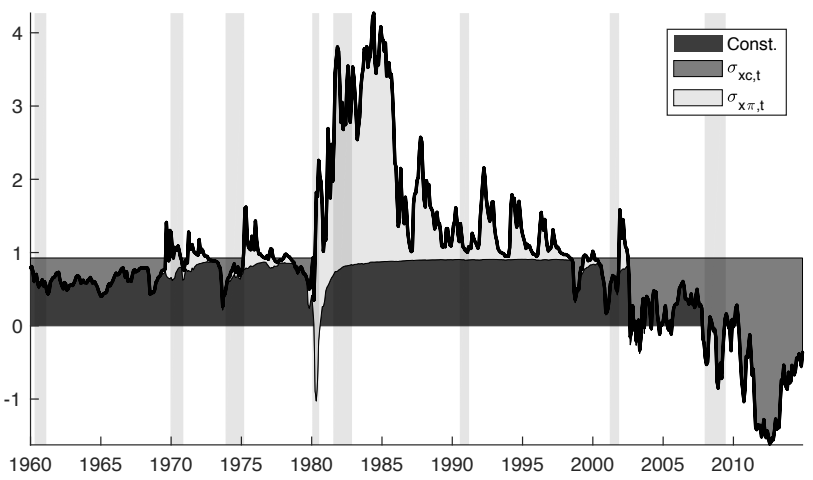

(c) Decomposition of the 5-Year Term Premium

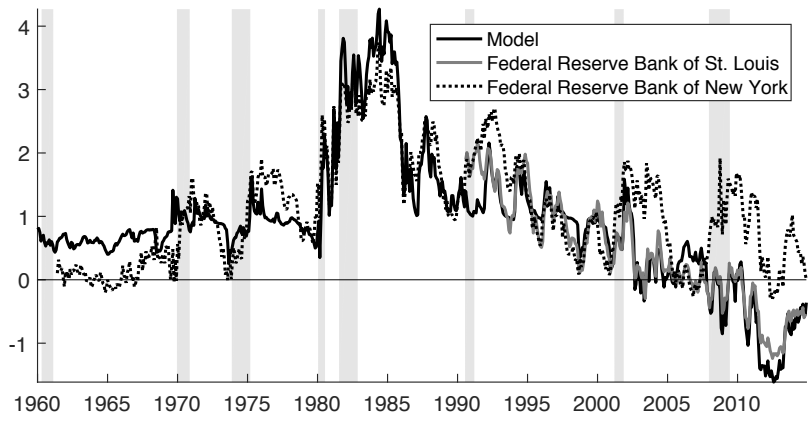

(b) 5-Year term premium: Different models

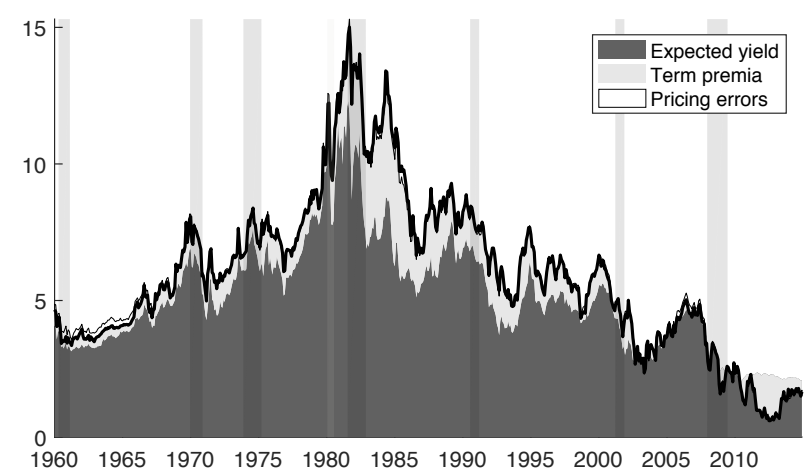

(d) 5-Year bond: Expected rates and term premium

\section{Figure 8 .}

Model-implied term premium. This figure shows the model-implied bond term premium. Panel (a) shows the posterior median term premium for different bond maturities. Panel (b) shows the posterior median model-implied term premium for the 5-Year bond together with the term premium estimated by Kim and Wright (2005) (gray line, Federal Reserve Bank of St. Louis) and Adrian et al. (2013) (black dashed line, Federal Reserve Bank of New York) for the same bond maturity. Panel (c) decomposes the posterior median of the 5-Year bond term premium into its different sources of risk. Panel (d) decomposes the 5-Year bond into expected average short-term rates and the term premium component. Light shaded bars represent recessions as defined by the National Bureau of Economic Research. The estimation sample is from 1960:M1 to 2014M12. 


\begin{tabular}{|c|c|c|c|c|c|c|c|c|}
\hline \multicolumn{3}{|c|}{ Prior Distribution } & \multicolumn{3}{|c|}{$\begin{array}{c}\text { Macro data } \\
\text { and bond prices }\end{array}$} & \multicolumn{3}{|c|}{ Macro data } \\
\hline Distr. & $5 \%$ & $95 \%$ & $5 \%$ & $50 \%$ & $95 \%$ & $5 \%$ & $50 \%$ & $95 \%$ \\
\hline \multicolumn{9}{|c|}{ Household Preferences } \\
\hline$B$ & 0.99680 & 0.99997 & - & 0.99900 & - & - & - & - \\
\hline$G$ & 0.65840 & 3.85547 & 1.50216 & 1.80972 & 2.00021 & - & - & - \\
\hline$G$ & 0.50489 & 17.34338 & 5.81235 & 8.01729 & 11.48261 & - & - & - \\
\hline \multicolumn{9}{|c|}{ Time preference shocks } \\
\hline$\rho_{\lambda} U$ & -0.89959 & 0.89988 & 0.94206 & 0.95506 & 0.96498 & - & - & - \\
\hline$\varphi_{\lambda} U$ & 0.14879 & 2.85044 & 0.21457 & 0.25022 & 0.31030 & - & - & - \\
\hline \multicolumn{9}{|c|}{ Consumption growth } \\
\hline$N$ & -0.00667 & 0.00984 & 0.00150 & 0.00164 & 0.00181 & 0.00011 & 0.00133 & 0.00203 \\
\hline$\sigma_{c} \quad I G$ & 0.00079 & 0.00611 & 0.00152 & 0.00162 & 0.00175 & 0.00124 & 0.00162 & 0.00207 \\
\hline$\varphi_{x c} U$ & 0.14874 & 2.84802 & 0.14997 & 0.19007 & 0.21532 & 0.14766 & 0.22489 & 0.34413 \\
\hline$\rho_{c c} U$ & -0.89986 & 0.89978 & 0.94056 & 0.96301 & 0.97471 & 0.89076 & 0.94603 & 0.97630 \\
\hline$\rho_{c \pi} U$ & -0.89934 & 0.89950 & -0.01585 & -0.00906 & -0.00505 & -0.04398 & -0.02090 & -0.00209 \\
\hline \multicolumn{9}{|c|}{ Inflation } \\
\hline$\mu_{\pi}$ & -0.00512 & 0.01132 & 0.00289 & 0.00314 & 0.00320 & 0.00122 & 0.00284 & 0.00403 \\
\hline$\varphi_{\pi} \quad I G$ & 0.14962 & 2.85048 & 0.80065 & 0.98890 & 1.25322 & 0.75878 & 0.98835 & 1.26624 \\
\hline$\varphi_{x \pi} I G$ & 0.14937 & 2.84935 & 0.08481 & 0.10016 & 0.12068 & 0.16288 & 0.22976 & 0.33315 \\
\hline$\rho_{\pi \pi} U$ & -0.89975 & 0.90004 & 0.98001 & 0.99005 & 0.99508 & 0.96405 & 0.98199 & 0.99485 \\
\hline \multicolumn{9}{|c|}{ Long-run volatility } \\
\hline$\rho_{h_{x c}} N^{T}$ & 0.71771 & 0.99215 & 0.97161 & 0.98049 & 0.99075 & 0.85926 & 0.97316 & 0.99478 \\
\hline$\sigma_{h_{x c}} I G$ & 0.00335 & 0.59617 & 0.11009 & 0.13958 & 0.15478 & 0.05908 & 0.11694 & 0.35035 \\
\hline$\rho_{h_{x \pi}} N^{T}$ & 0.71793 & 0.99224 & 0.95141 & 0.96183 & 0.97563 & 0.70757 & 0.81366 & 0.89535 \\
\hline$\sigma_{h_{x \pi}} I G$ & 0.00333 & 0.59384 & 0.26966 & 0.30004 & 0.32003 & 0.21978 & 0.30999 & 0.41028 \\
\hline \multicolumn{9}{|c|}{ Short-run Volatility } \\
\hline$\rho_{h_{c}} N^{T}$ & 0.71771 & 0.99215 & 0.87385 & 0.97301 & 0.98978 & 0.85926 & 0.97316 & 0.99478 \\
\hline$\sigma_{h_{c}} I G$ & 0.00335 & 0.59617 & 0.07027 & 0.11690 & 0.24964 & 0.05908 & 0.11694 & 0.35035 \\
\hline$\rho_{h_{\pi}} N^{T}$ & 0.71793 & 0.99224 & 0.75864 & 0.81348 & 0.87055 & 0.70757 & 0.81366 & 0.89535 \\
\hline$\sigma_{h_{\pi}} I G$ & 0.00333 & 0.59384 & 0.24959 & 0.31007 & 0.38971 & 0.21978 & 0.30999 & 0.41028 \\
\hline \multicolumn{9}{|c|}{ Consumption measurement errors } \\
\hline$\sigma_{\epsilon} \quad I G$ & 0.00079 & 0.00612 & 0.00133 & 0.00145 & 0.00165 & 0.00129 & 0.00155 & 0.00175 \\
\hline$\sigma_{\epsilon}^{q r t} I G$ & 0.00067 & 0.03898 & 0.00062 & 0.00080 & 0.00936 & 0.00055 & 0.00084 & 0.00111 \\
\hline
\end{tabular}

Table 1.

Prior and posterior model estimates. This table reports the 5 and 95 percentiles of the prior distribution of the model parameter along with the 5, 50, and 95 percentiles of their posterior distribution. We present the posterior distribution with (Macro data and bond prices) and without (Macro data) bond prices included in the estimation. The assumed prior distributions are the following: $B$ - beta, $G$-gamma, $I G$ - inverse gamma, $N$-normal , $N^{T}$ - truncated normal outside of the $(-1,1)$ interval and $U$ - uniform. We assume that the measurement errors of consumption average out every quarter. We also assume a common stochastic volatility process (i.e., $h_{c, t}=h_{x c, t}$ and $\left.h_{\pi, t}=h_{x \pi, t}\right)$ in the estimation with only considering macro data. The estimation sample is from 1960:M1 to 2014M12. 


\begin{tabular}{cccccccc}
\hline & \multicolumn{3}{c}{$\begin{array}{c}\text { Macro data } \\
\text { and bond prices }\end{array}$} & & \multicolumn{3}{c}{ Macro data } \\
\cline { 2 - 4 } \cline { 6 - 8 }$E\left[\sigma_{i, t}\right]$ & $5 \%$ & $50 \%$ & $95 \%$ & & $5 \%$ & $50 \%$ & $95 \%$ \\
\hline \multicolumn{4}{c}{ Unconditional long-run volatilities } \\
\hline$E\left[\sigma_{c, t}\right]$ & 0.00160 & 0.00184 & 0.00356 & & 0.00157 & 0.00191 & 0.00248 \\
$E\left[\sigma_{\pi, t}\right]$ & 0.00137 & 0.00185 & 0.00252 & & 0.00171 & 0.00184 & 0.00198 \\
$E\left[\sigma_{x c, t}\right]$ & 0.00037 & 0.00040 & 0.00045 & & 0.00031 & 0.00043 & 0.00062 \\
$E\left[\sigma_{x \pi, t}\right]$ & 0.00024 & 0.00030 & 0.00043 & & 0.00034 & 0.00043 & 0.00055 \\
\hline \hline
\end{tabular}

Table 2.

Unconditional mean volatilities This table reports the 5, 50, and 95 percentiles of the posterior distribution of unconditional mean of the volatility process $E\left[\sigma_{i, t}\right]=\varphi_{i} \sigma_{c} \exp \left(0.5 \frac{\sigma_{h_{i}}^{2}}{1-\rho_{h_{i}}^{2}}\right)$ implied by the posterior distribution of the model parameters. We present the posterior distribution with (Macro data and bond prices) and without (Macro data) bond prices included in the estimation. The estimation sample is from 1960:M1 to 2014M12. 


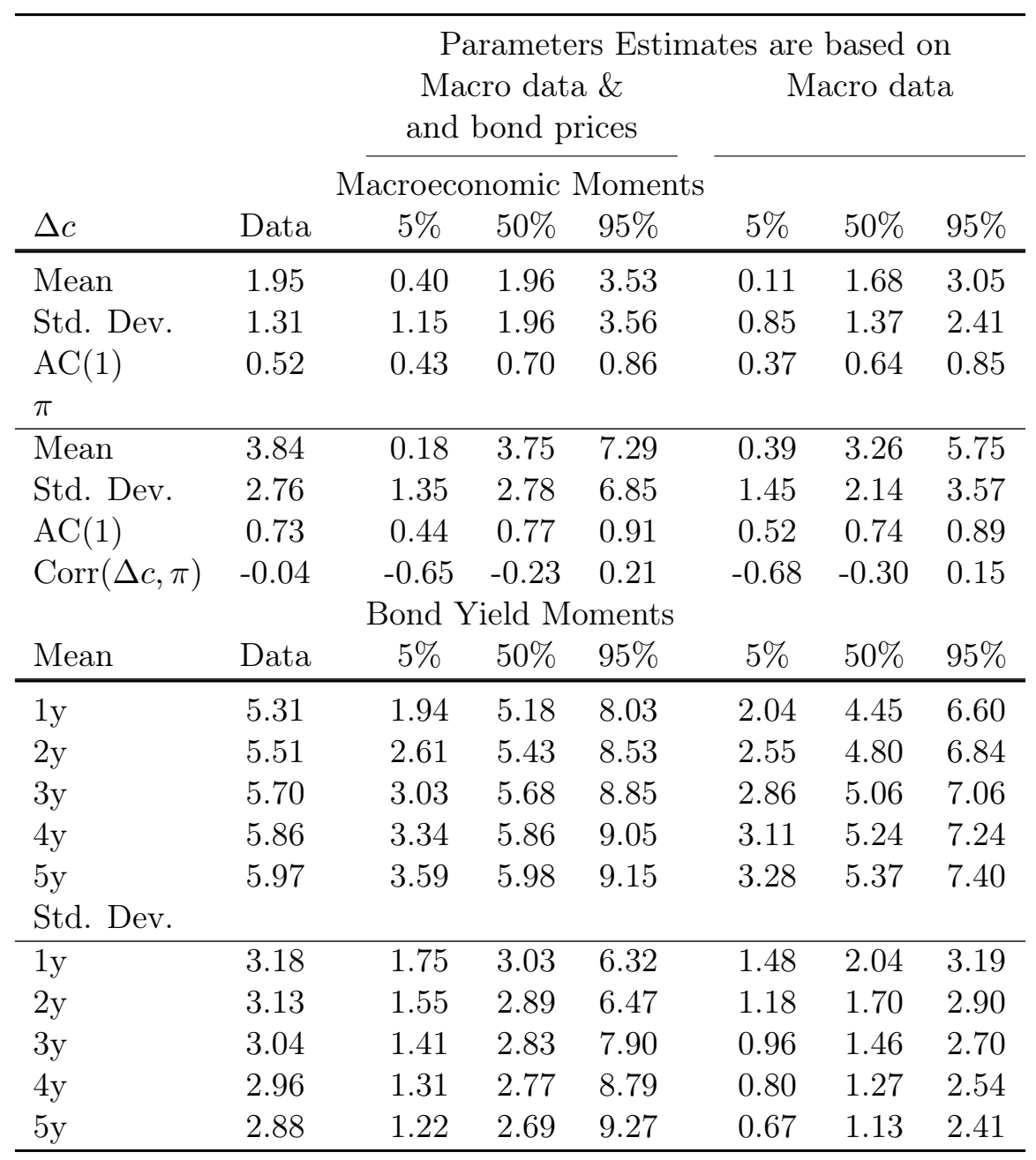

Table 3.

Moments of macroeconomic variables and bond yields: Data and model. This table reports descriptive statistics for consumption growth, inflation, and nominal yields. We included the sample data moments and the 5, 50, and 95 percentiles of the model-implied moments based on 10,000 parameter draws of the posterior distribution with and without bond prices in the estimation. We simulated the series for 660 periods, which equals the number of periods in the sample. To compute the bond yield moments with only considering macroeconomic data, we set the preference parameters $\delta, \psi, \gamma, \rho_{\lambda}$ and $\varphi_{\lambda}$ to the posterior median estimates from Table 1. The estimation sample is from $1960 \mathrm{M} 1$ to $2014 \mathrm{M} 12$. 


\begin{tabular}{|c|c|c|c|c|c|c|c|c|c|}
\hline & \multicolumn{3}{|c|}{ SD Inflation News } & \multicolumn{3}{|c|}{ SD Yield News } & \multicolumn{3}{|c|}{ Inflation Variance Ratios } \\
\hline & $5 \%$ & $50 \%$ & $95 \%$ & $5 \%$ & $50 \%$ & $95 \%$ & $5 \%$ & $50 \%$ & $95 \%$ \\
\hline \multicolumn{10}{|c|}{ Without Stochastic Volatility } \\
\hline $1 \mathrm{y}$ & 0.15 & 0.17 & 0.19 & 0.33 & 0.36 & 0.41 & 0.16 & 0.21 & 0.28 \\
\hline $2 y$ & 0.14 & 0.16 & 0.18 & 0.29 & 0.33 & 0.37 & 0.17 & 0.24 & 0.32 \\
\hline $3 y$ & 0.14 & 0.15 & 0.17 & 0.27 & 0.30 & 0.34 & 0.19 & 0.26 & 0.35 \\
\hline $4 y$ & 0.13 & 0.15 & 0.16 & 0.25 & 0.28 & 0.32 & 0.19 & 0.27 & 0.36 \\
\hline $5 y$ & 0.13 & 0.14 & 0.16 & 0.24 & 0.27 & 0.30 & 0.20 & 0.27 & 0.37 \\
\hline \multicolumn{10}{|c|}{ With Stochastic Volatility } \\
\hline $1 \mathrm{y}$ & 0.15 & 0.18 & 0.21 & 0.35 & 0.41 & 0.48 & 0.20 & 0.32 & 0.62 \\
\hline $2 y$ & 0.14 & 0.17 & 0.21 & 0.30 & 0.35 & 0.41 & 0.23 & 0.34 & 0.61 \\
\hline $3 y$ & 0.14 & 0.16 & 0.20 & 0.27 & 0.31 & 0.36 & 0.22 & 0.33 & 0.56 \\
\hline $4 y$ & 0.13 & 0.15 & 0.19 & 0.25 & 0.29 & 0.33 & 0.22 & 0.33 & 0.54 \\
\hline $5 y$ & 0.13 & 0.15 & 0.18 & 0.24 & 0.27 & 0.31 & 0.23 & 0.34 & 0.53 \\
\hline
\end{tabular}

Table 4.

Inflation variance ratios: Statistical model. This table reports the posterior distribution of the standard deviation of shocks to average expected inflation over the life of the bond (SD Inflation News), standard deviation of yield shocks (SD Yield News), and the inflation variance ratios. We include the 5, 50, and 95 percentiles based on 10,000 parameter draws of the posterior distribution from a statistical model that does not include (top panel) and does include (bottom panel) stochastic volatility. Standard deviations are in annualized percent/month. To compute these estimates, we draw the parameters from their posterior distribution and simulate 10,000 data sets of 555 periods. 


\begin{tabular}{|c|c|c|c|c|c|c|c|c|c|c|}
\hline \multicolumn{5}{|c|}{$\begin{array}{c}\text { With Time Preference Shocks } \\
\text { SD Inflation News }\end{array}$} & \multicolumn{3}{|c|}{ SD Yield News } & \multicolumn{3}{|c|}{ Inflation Variance Ratios } \\
\hline & \multicolumn{2}{|c|}{ Data } & \multicolumn{2}{|r|}{ Model } & \multicolumn{2}{|c|}{ Data } & Model & \multicolumn{2}{|c|}{ Data } & Model \\
\hline & $\mathrm{w} / \mathrm{o} \mathrm{SV}$ & $\mathrm{w} / \mathrm{SV}$ & Pop. & $5 \% \quad 50 \% 95 \%$ & $\mathrm{w} / \mathrm{o} \mathrm{SV}$ & $\mathrm{w} / \mathrm{SV}$ & Pop. $5 \% \quad 50 \% 95 \%$ & $\mathrm{w} / \mathrm{o} \mathrm{S}$ & $\mathrm{w} / \mathrm{SV}$ & Pop. $5 \% 50 \% 95 \%$ \\
\hline $1 \mathrm{y}$ & 0.17 & 0.18 & 0.18 & $\begin{array}{lll}0.16 & 0.32 & 0.78\end{array}$ & 0.37 & 0.41 & $\begin{array}{lllll}0.44 & 0.46 & 0.60 & 0.99\end{array}$ & 0.21 & 0.32 & $\begin{array}{llll}0.17 & 0.14 & 0.28 & 0.49\end{array}$ \\
\hline $2 \mathrm{y}$ & 0.16 & 0.17 & 0.17 & $\begin{array}{lll}0.15 & 0.30 & 0.70\end{array}$ & 0.33 & 0.35 & $\begin{array}{llll}0.36 & 0.38 & 0.50 & 0.84\end{array}$ & 0.24 & 0.33 & $\begin{array}{llll}0.23 & 0.17 & 0.33 & 0.55\end{array}$ \\
\hline $3 y$ & 0.15 & 0.16 & 0.16 & $\begin{array}{lll}0.14 & 0.28 & 0.64\end{array}$ & 0.30 & 0.31 & $\begin{array}{llll}0.31 & 0.32 & 0.44 & 0.74\end{array}$ & 0.26 & 0.35 & $\begin{array}{llll}0.28 & 0.20 & 0.37 & 0.60\end{array}$ \\
\hline $4 y$ & 0.15 & 0.16 & 0.16 & $\begin{array}{lll}0.13 & 0.27 & 0.60\end{array}$ & 0.28 & 0.29 & $\begin{array}{llll}0.27 & 0.28 & 0.39 & 0.66\end{array}$ & 0.27 & 0.33 & $\begin{array}{llll}0.34 & 0.22 & 0.41 & 0.65\end{array}$ \\
\hline $5 y$ & 0.14 & 0.15 & 0.15 & $\begin{array}{lll}0.12 & 0.25 & 0.56\end{array}$ & 0.27 & 0.27 & $\begin{array}{llll}0.24 & 0.24 & 0.35 & 0.60\end{array}$ & 0.27 & 0.33 & $\begin{array}{llll}0.39 & 0.25 & 0.45 & 0.69\end{array}$ \\
\hline
\end{tabular}

\section{Table 5.}

Inflation variance ratios: Macro-finance and statistical model. This table reports the posterior distribution of the standard deviation of shocks to average expected inflation over the life of the bond (SD Inflation News), standard deviation of yield shocks (SD Yield News), and the inflation variance ratios. We include the 5, 50, and 95 percentiles based on 10,000 parameter draws of the posterior distribution from a macro-finance model that does include time preference shocks. Pop. denotes the population estimates. We also report the posterior median values computed from the statistical model that does not assume (w/o SV) and does assume (w/ SV) stochastic volatility. Standard deviations are in annualized percent/month. To compute these estimates, we draw the parameters from their posterior distribution and simulate 10,000 data sets of 660 (555) periods for the macro-finance model (statistical models). 


\begin{tabular}{|c|c|c|c|c|c|c|c|}
\hline \multirow[b]{3}{*}{$n$} & \multirow[b]{3}{*}{ Data } & \multicolumn{6}{|c|}{ Parameters Estimates are based on } \\
\hline & & \multicolumn{3}{|c|}{$\begin{array}{l}\text { Macro data } \\
\text { and bond prices }\end{array}$} & \multicolumn{3}{|c|}{ Macro data } \\
\hline & & $5 \%$ & $50 \%$ & $95 \%$ & $5 \%$ & $50 \%$ & $95 \%$ \\
\hline \multicolumn{8}{|c|}{ Campbell-Shiller Regression: Slope } \\
\hline $2 y$ & -0.67 & -1.59 & -0.37 & 1.31 & 0.32 & 1.00 & 1.83 \\
\hline $3 y$ & -1.07 & -2.32 & -0.72 & 1.28 & 0.40 & 1.12 & 2.05 \\
\hline $4 y$ & -1.47 & -3.09 & -0.99 & 1.31 & 0.43 & 1.15 & 2.12 \\
\hline $5 y$ & -1.50 & -3.88 & -1.18 & 1.39 & 0.45 & 1.16 & 2.15 \\
\hline \multicolumn{8}{|c|}{ Cochrane-Piazzesi Regression: Slope } \\
\hline $2 y$ & 0.45 & 0.32 & 0.42 & 0.52 & 0.50 & 0.60 & 0.71 \\
\hline $3 y$ & 0.86 & 0.76 & 0.83 & 0.90 & 0.86 & 0.94 & 1.03 \\
\hline $4 y$ & 1.25 & 1.18 & 1.21 & 1.23 & 1.08 & 1.16 & 1.23 \\
\hline $5 y$ & 1.44 & 1.40 & 1.54 & 1.69 & 1.15 & 1.30 & 1.46 \\
\hline \multicolumn{8}{|c|}{ Cochrane-Piazzesi Regression: $R^{2}$} \\
\hline $2 y$ & 0.21 & 0.06 & 0.32 & 0.83 & 0.00 & 0.03 & 0.10 \\
\hline $3 y$ & 0.23 & 0.09 & 0.36 & 0.80 & 0.00 & 0.03 & 0.10 \\
\hline $4 y$ & 0.26 & 0.10 & 0.36 & 0.72 & 0.00 & 0.03 & 0.10 \\
\hline $5 y$ & 0.24 & 0.10 & 0.35 & 0.66 & 0.00 & 0.03 & 0.10 \\
\hline
\end{tabular}

Table 6.

Bond risk premia: Data and model. This table reports bond return predictability evidence. We included the 5, 50, and 95 percentiles of the model-implied estimates based on two different regressions as well as the estimates obtained from observed bond yields. The model-implied bond yield series is based on 10,000 parameter draws of the posterior distribution with and without considering bond prices in the estimation. We simulated the series for 660 periods, which equals the number of periods in the sample. To compute the model-implied bond yields based only on macro data, we set the preference parameters $\delta, \psi, \gamma, \rho_{\lambda}$ and $\varphi_{\lambda}$ to the posterior median estimates from Table 1. For the Campbell-Shiller regression we report the slope, $\beta_{n}$, from the following regression: $y_{t+12, n-12}-y_{t, n}=\alpha_{n}+\beta_{n} \frac{12}{n-12}\left(y_{t, n}-y_{t, 12}\right)+\epsilon_{t+12} \quad$ for $n \in\{24,36,48,60\}$. For the Cochrane-Piazzesi regression wereport the slope, $b_{n}$, and $R^{2}$ from the following regression: $r x_{r \rightarrow t+12, n}^{\$}=a_{n}+b_{n} \hat{r} x_{t}+\epsilon_{t+1, n}$ where $\hat{r} x_{t}$ is the fitted value from $\frac{1}{4} \sum_{n=2 y}^{5 y} r x_{r \rightarrow t+1, n}^{\$}=\gamma_{0}+$ $\gamma_{1} f_{t, 1 y}^{\$}+\gamma_{2} f_{t, 2 y}^{\$}+\gamma_{3} f_{t, 3 y}^{\$}+\gamma_{4} f_{t, 4 y}^{\$}+\gamma_{5} f_{t, 5 y}^{\$}+\epsilon_{t+1}$. $\quad r x_{r \rightarrow t+1, n}^{\$}$ denotes the excess log return on buying an $n$ period bond at time $t$ and selling it at time $t+1$ as an $n-1$ period bond defined by $r x_{r \rightarrow t+1, n}^{\$}=n y_{t, n}^{\$}-(n-1) y_{t+1, n-1}^{\$}-y_{t, 1}^{\$}$ for $n \in\{24,36,48,60\} . f^{\$}$ denotes the corresponding forward rates. The estimation sample is from 1960M1 to 2014M12. 


\begin{tabular}{|c|c|c|c|c|c|c|c|c|c|c|c|c|c|}
\hline \multicolumn{14}{|c|}{ Macro data } \\
\hline & \multirow{2}{*}{\multicolumn{12}{|c|}{ Posterior Distribution }} & \multirow{3}{*}{$\ln p\left(Y^{o}\right)$} \\
\hline & \multicolumn{4}{|c|}{$\rho_{c c}$} & \multicolumn{2}{|c|}{$\rho_{c \pi}$} & & & & & & & \\
\hline & $5 \%$ & $50 \%$ & $95 \%$ & Width & $5 \%$ & $50 \%$ & $95 \%$ & Width & $5 \%$ & $50 \%$ & $95 \%$ & Width & \\
\hline (a) No Me. & -0.286 & -0.206 & -0.130 & 0.156 & -0.223 & -0.128 & -0.0392 & 0.184 & 0.849 & 0.912 & 0.963 & 0.1139 & 5855 \\
\hline (b) Me. & 0.828 & 0.892 & 0.941 & 0.112 & -0.041 & -0.019 & -0.0008 & 0.040 & 0.873 & 0.9363 & 0.972 & 0.098 & 5870 \\
\hline (c) Me. Sv. & 0.890 & 0.946 & 0.976 & 0.085 & -0.044 & -0.021 & -0.002 & 0.041 & 0.964 & 0.9820 & 0.994 & 0.030 & 6055 \\
\hline
\end{tabular}

\section{Table B1.}

Posterior estimates of the persistence parameters. This table reports the 5, 50, and 95 percentiles of the posterior distribution for the persistent parameters $\rho_{c c}, \rho_{c \pi}$ and $\rho_{\pi \pi}$ for the monthly version of the model. We also include the $90 \%$ confidence width and the log marginal data density. We consider three different specifications of the process of consumption growth and inflation. No Me., consumption growth is measured without any errors, (i.e., $c_{t}^{o}=c_{t}$ ), and homoskedastic innovations; Me. assumes the measurement error model of consumption; Me. Sv., assumes the measurement error model of consumption and short- and long-run stochastic volatility imposing a common stochastic volatility process (i.e. $h_{c, t}=h_{x c, t}$ and $h_{\pi, t}=h_{x \pi, t}$ ). The estimation sample is from 1960:M1 to 2014M12.

\begin{tabular}{|c|c|c|c|c|c|c|c|c|c|c|c|}
\hline & \multicolumn{2}{|c|}{ Data } & \multirow{2}{*}{$\begin{array}{c}\text { Posterior Median } \\
\rho_{\lambda}=0.95, \sigma_{\lambda}=0.25\end{array}$} & \multicolumn{4}{|c|}{ Different values for $\rho_{\lambda}$} & \multicolumn{4}{|c|}{ Different values for $\sigma_{\lambda}$} \\
\hline & $\mathrm{w} / \mathrm{o} \mathrm{SV}$ & $\mathrm{w} / \mathrm{SV}$ & & 0.50 & 0.90 & 0.99 & 0.999 & 0.02 & 0.12 & 0.42 & 0.62 \\
\hline \multicolumn{12}{|c|}{ A) Inflation Variance Ratios } \\
\hline $1 \mathrm{y}$ & 0.21 & 0.32 & 0.17 & 0.53 & 0.36 & 0.08 & 0.04 & 0.53 & 0.36 & 0.08 & 0.04 \\
\hline $2 y$ & 0.24 & 0.33 & 0.23 & 0.60 & 0.44 & 0.11 & 0.05 & 0.60 & 0.44 & 0.11 & 0.05 \\
\hline $3 y$ & 0.26 & 0.35 & 0.28 & 0.65 & 0.51 & 0.14 & 0.07 & 0.65 & 0.51 & 0.14 & 0.07 \\
\hline $4 y$ & 0.27 & 0.33 & 0.34 & 0.70 & 0.56 & 0.18 & 0.09 & 0.70 & 0.56 & 0.18 & 0.09 \\
\hline $5 y$ & 0.27 & 0.33 & 0.39 & 0.73 & 0.61 & 0.21 & 0.11 & 0.73 & 0.61 & 0.21 & 0.11 \\
\hline \multicolumn{12}{|c|}{ B) Slope } \\
\hline Mean & 0 . & & 0.83 & 0.16 & 0.40 & 2.03 & 4.21 & 0.15 & 0.28 & 2.05 & 4.18 \\
\hline \multicolumn{12}{|c|}{ C) Campbell-Shiller Regression Slope } \\
\hline $2 \mathrm{y}$ & -0 & & -0.33 & -0.69 & -0.58 & -0.03 & 0.29 & -0.62 & -0.55 & 0.02 & 0.35 \\
\hline $3 y$ & -1 & & -0.65 & -1.09 & -0.98 & -0.25 & 0.17 & -1.02 & -0.94 & -0.19 & 0.20 \\
\hline $4 y$ & -1 & & -0.88 & -1.44 & -1.29 & -0.43 & 0.06 & -1.34 & -1.20 & -0.33 & 0.13 \\
\hline $5 y$ & -1 & & -1.03 & -1.68 & -1.52 & -0.54 & 0.00 & -1.53 & -1.41 & -0.45 & 0.08 \\
\hline
\end{tabular}

\section{Table B2.}

The importance of the time preference shocks in the nominal yield curve. This table shows data and model-implied inflation variance ratios, slope of the term structure, as well as the Campbell-Shiller regression slope. For the inflation variance ratios we report population values. For the slope and Campbell-Shiller Regression Slope we report the $50 \%$ percentile of the finite sample distribution. In all cases we used the posterior median of the parameter estimates. We are also simulating the moments by gradually varying $\rho_{\lambda}$ and $\sigma_{\lambda}$. The column labeled "Data" shows the moments based on observed data. The column labeled "Posterior Median" shows the modelimplied moments evaluated at the posterior median parameters. These model-implied moments differ slightly from the ones reported in the actual paper because in this exercise we are not taking parameter uncertainty into account. 
Posterior Median Estimates

\begin{tabular}{|c|c|c|c|c|c|c|c|c|c|c|}
\hline & \multicolumn{5}{|c|}{ Without Stochastic Volatility } & \multicolumn{5}{|c|}{ With Stochastic Volatility } \\
\hline & $1 \mathrm{y}$ & $2 \mathrm{y}$ & $3 y$ & $4 y$ & $5 y$ & $1 \mathrm{y}$ & $2 \mathrm{y}$ & $3 y$ & $4 y$ & $5 y$ \\
\hline \multicolumn{11}{|c|}{ Inflation and Bond Yields } \\
\hline$\mu$ & $4.28 \mathrm{e}-03$ & $4.19 \mathrm{e}-03$ & $4.23 \mathrm{e}-03$ & $4.22 \mathrm{e}-03$ & $4.23 \mathrm{e}-03$ & $4.07 \mathrm{e}-03$ & $3.90 \mathrm{e}-03$ & $3.70 \mathrm{e}-03$ & $3.80 \mathrm{e}-03$ & $4.14 \mathrm{e}-03$ \\
\hline$\sigma_{\pi}$ & $2.70 \mathrm{e}-03$ & $2.69 \mathrm{e}-03$ & $2.70 \mathrm{e}-03$ & $2.68 \mathrm{e}-03$ & $2.70 \mathrm{e}-03$ & $1.97 \mathrm{e}-03$ & $1.97 \mathrm{e}-03$ & $1.96 \mathrm{e}-03$ & $1.99 \mathrm{e}-03$ & $1.96 \mathrm{e}-03$ \\
\hline$\rho$ & 0.99 & 0.99 & 0.99 & 0.99 & 0.99 & 0.99 & 0.99 & 0.99 & 0.99 & 0.99 \\
\hline$\sigma_{x}$ & $1.46 \mathrm{e}-04$ & $1.46 \mathrm{e}-04$ & $1.46 \mathrm{e}-04$ & $1.47 \mathrm{e}-04$ & $1.47 \mathrm{e}-04$ & $1.16 \mathrm{e}-04$ & $1.09 \mathrm{e}-04$ & $1.12 \mathrm{e}-04$ & $1.09 \mathrm{e}-04$ & $1.18 \mathrm{e}-04$ \\
\hline$\sigma_{y}$ & $3.04 \mathrm{e}-04$ & $2.72 \mathrm{e}-04$ & $2.49 \mathrm{e}-04$ & $2.37 \mathrm{e}-04$ & $2.23 \mathrm{e}-04$ & $2.62 \mathrm{e}-04$ & $2.43 \mathrm{e}-04$ & $2.31 \mathrm{e}-04$ & $2.22 \mathrm{e}-04$ & $2.13 \mathrm{e}-04$ \\
\hline $\operatorname{corr}_{x, y}$ & 0.51 & 0.52 & 0.51 & 0.52 & 0.52 & 0.51 & 0.52 & 0.51 & 0.52 & 0.52 \\
\hline \multicolumn{11}{|c|}{ Stochastic volatilities } \\
\hline$\rho_{h_{\pi}}$ & - & - & - & - & - & 0.90 & 0.90 & 0.90 & 0.90 & 0.90 \\
\hline$\sigma_{h_{\pi}}$ & - & - & - & - & & $5.43 \mathrm{e}-02$ & $5.57 \mathrm{e}-02$ & $5.83 \mathrm{e}-02$ & $5.49 \mathrm{e}-02$ & $5.69 \mathrm{e}-02$ \\
\hline$\rho_{h_{x}}$ & - & - & - & - & - & 0.96 & 0.94 & 0.96 & 0.95 & 0.95 \\
\hline$\sigma_{h_{x}}$ & - & - & - & - & - & $1.99 \mathrm{e}-02$ & $3.92 \mathrm{e}-02$ & $2.19 \mathrm{e}-02$ & $3.35 \mathrm{e}-02$ & $2.59 \mathrm{e}-02$ \\
\hline$\rho_{h_{y}}$ & - & - & - & - & - & 0.98 & 0.98 & 0.97 & 0.95 & 0.91 \\
\hline$\sigma_{h_{y}}$ & - & - & - & - & - & $9.46 \mathrm{e}-03$ & $7.36 \mathrm{e}-03$ & $5.68 \mathrm{e}-03$ & $5.23 \mathrm{e}-03$ & $5.66 \mathrm{e}-03$ \\
\hline \multicolumn{11}{|c|}{ Measurement error SPF } \\
\hline$\sigma_{C P I}^{s}$ & $4.61 \mathrm{e}-04$ & $4.63 \mathrm{e}-04$ & $4.63 \mathrm{e}-04$ & $4.61 \mathrm{e}-04$ & $4.62 \mathrm{e}-04$ & $4.64 \mathrm{e}-04$ & $4.66 \mathrm{e}-04$ & $4.70 \mathrm{e}-04$ & $4.69 \mathrm{e}-04$ & $4.63 \mathrm{e}-04$ \\
\hline$\sigma_{P G D P}^{s}$ & $1.27 \mathrm{e}-03$ & $1.27 \mathrm{e}-03$ & $1.27 \mathrm{e}-03$ & $1.26 \mathrm{e}-03$ & $1.27 \mathrm{e}-03$ & $1.23 \mathrm{e}-03$ & $1.21 \mathrm{e}-03$ & $1.19 \mathrm{e}-03$ & $1.21 \mathrm{e}-03$ & $1.24 \mathrm{e}-03$ \\
\hline $\ln p\left(Y^{o}\right)$ & 10053 & 10082 & 10102 & 10112 & 10124 & 10075 & 10104 & 10125 & 10135 & 10145 \\
\hline
\end{tabular}

\section{Table C1.}

Posterior median estimates from the statistical models. This table reports the 50 percentiles of their posterior distribution of the statistical model that does not assume and does assume stochastic volatility. We assume non-informative priors for the model parameters. The statistical model assumes that inflation is the sum of a constant and an $\mathrm{AR}(1)$ component. Shocks to bond yields are computed assuming they are martingales. See main text for details. The estimation sample is from 1968M10 to 2014M12. 


\begin{tabular}{|c|c|c|c|c|c|c|}
\hline & \multicolumn{6}{|c|}{ Quarterly Frequency } \\
\hline & \multicolumn{3}{|c|}{ Homoskedastic } & \multicolumn{3}{|c|}{ Heteroskedastic } \\
\hline & $5 \%$ & $50 \%$ & $95 \%$ & $5 \%$ & $50 \%$ & $95 \%$ \\
\hline (a) & \multicolumn{6}{|c|}{$\begin{array}{c}\pi \text { - SPF for observed quarter CPI } \\
\text { Time period 1981Q3-2014Q4 } \\
\end{array}$} \\
\hline $1 \mathrm{y}$ & 0.075 & 0.103 & 0.142 & 0.164 & 0.236 & 0.327 \\
\hline $2 y$ & 0.082 & 0.108 & 0.145 & 0.164 & 0.230 & 0.333 \\
\hline $3 y$ & 0.091 & 0.119 & 0.163 & 0.167 & 0.225 & 0.325 \\
\hline $4 y$ & 0.087 & 0.121 & 0.168 & 0.145 & 0.203 & 0.292 \\
\hline $5 y$ & 0.084 & 0.120 & 0.167 & 0.136 & 0.193 & 0.274 \\
\hline $\ln p\left(Y^{o}\right)$ & \multicolumn{3}{|c|}{7088.304} & \multicolumn{3}{|c|}{7422.010} \\
\hline \multirow[t]{2}{*}{ (b) } & \multicolumn{6}{|c|}{$\pi-\mathrm{PGDP}$} \\
\hline & \multicolumn{6}{|c|}{ Time period 1968Q1-2014Q4 } \\
\hline $1 \mathrm{y}$ & 0.095 & 0.124 & 0.162 & 0.188 & 0.274 & 0.394 \\
\hline $2 y$ & 0.102 & 0.134 & 0.174 & 0.208 & 0.292 & 0.420 \\
\hline $3 y$ & 0.102 & 0.139 & 0.182 & 0.198 & 0.285 & 0.402 \\
\hline $4 y$ & 0.101 & 0.137 & 0.181 & 0.165 & 0.242 & 0.352 \\
\hline $5 y$ & 0.097 & 0.131 & 0.174 & 0.141 & 0.211 & 0.307 \\
\hline $\ln p\left(Y^{o}\right)$ & \multicolumn{3}{|c|}{9499.774} & \multicolumn{3}{|c|}{10115.738} \\
\hline (c) & \multicolumn{6}{|c|}{$\begin{array}{c}\pi \text { - SPF for current quarter CPI } \\
\text { Time period 1981Q3-2014Q4 } \\
\end{array}$} \\
\hline $1 y$ & 0.078 & 0.104 & 0.137 & 0.163 & 0.240 & 0.342 \\
\hline $2 y$ & 0.080 & 0.110 & 0.145 & 0.161 & 0.235 & 0.341 \\
\hline $3 y$ & 0.090 & 0.120 & 0.158 & 0.157 & 0.226 & 0.328 \\
\hline $4 y$ & 0.087 & 0.122 & 0.163 & 0.151 & 0.213 & 0.299 \\
\hline $5 y$ & 0.087 & 0.118 & 0.158 & 0.139 & 0.201 & 0.288 \\
\hline $\ln p\left(Y^{o}\right)$ & \multicolumn{3}{|c|}{7197.171} & \multicolumn{3}{|c|}{7558.242} \\
\hline
\end{tabular}

Table C2.

Unconditional inflation variance ratios. This table reports the population values of inflation variance ratios for three different measures of inflation forecasts. The inflation variance ratio is computed as the ratio of the variance of shocks to expected inflation to the variance of yield shocks. We include the 5, 50, and 95 percentiles of the moments based on 10,000 parameter draws of the posterior distribution. The model assumes that inflation is the sum of a constant and an $\operatorname{AR}(1)$ component. Shocks to the yields are computed assuming they are martingales. The table reports inflation variance ratio under the assumption of homoskedastic and heteroskedastic innovations. 